\title{
Fungal Biodiversity in Salt Marsh Ecosystems
}

\author{
Mark S. Calabon ${ }^{1,2}{ }^{\mathbb{D}}$, E. B. Gareth Jones ${ }^{3}$, Itthayakorn Promputtha ${ }^{4,5}$ and Kevin D. Hyde ${ }^{1,2,4,6, *}$ \\ 1 Center of Excellence in Fungal Research, Mae Fah Luang University, Chiang Rai 57100, Thailand; \\ mscalabon@up.edu.ph \\ 2 School of Science, Mae Fah Luang University, Chiang Rai 57100, Thailand \\ 3 Department of Botany and Microbiology, College of Science, King Saud University, P.O. Box 2455, \\ Riyadh 11451, Saudi Arabia; torperadgj@gmail.com \\ 4 Department of Biology, Faculty of Science, Chiang Mai University, Chiang Mai 50200, Thailand; \\ itthayakorn.p@cmu.ac.th \\ 5 Environmental Science Research Center, Faculty of Science, Chiang Mai University, \\ Chiang Mai 50200, Thailand \\ 6 Innovative Institute of Plant Health, Zhongkai University of Agriculture and Engineering, \\ Guangzhou 510225, China \\ * Correspondence: kdhyde3@gmail.com
}

check for

updates

Citation: Calabon, M.S.; Jones, E.B.G.; Promputtha, I.; Hyde, K.D. Fungal Biodiversity in Salt Marsh

Ecosystems. J. Fungi 2021, 7, 648. https://doi.org/10.3390/jof7080648

Academic Editor: Wei Li

Received: 26 June 2021

Accepted: 30 July 2021

Published: 9 August 2021

Publisher's Note: MDPI stays neutral with regard to jurisdictional claims in published maps and institutional affiliations.

Copyright: (c) 2021 by the authors. Licensee MDPI, Basel, Switzerland. This article is an open access article distributed under the terms and conditions of the Creative Commons Attribution (CC BY) license (https:// creativecommons.org/licenses/by/ $4.0 /)$.

\begin{abstract}
This review brings together the research efforts on salt marsh fungi, including their geographical distribution and host association. A total of 486 taxa associated with different hosts in salt marsh ecosystems are listed in this review. The taxa belong to three phyla wherein Ascomycota dominates the taxa from salt marsh ecosystems accounting for $95.27 \%$ (463 taxa). The Basidiomycota and Mucoromycota constitute 19 taxa and four taxa, respectively. Dothideomycetes has the highest number of taxa, which comprises 47.12\% (229 taxa), followed by Sordariomycetes with 167 taxa $(34.36 \%)$. Pleosporales is the largest order with 178 taxa recorded. Twenty-seven genera under 11 families of halophytes were reviewed for its fungal associates. Juncus roemerianus has been extensively studied for its associates with 162 documented taxa followed by Phragmites australis (137 taxa) and Spartina alterniflora (79 taxa). The highest number of salt marsh fungi have been recorded from Atlantic Ocean countries wherein the USA had the highest number of species recorded (232 taxa) followed by the UK (101 taxa), the Netherlands (74 taxa), and Argentina (51 taxa). China had the highest number of salt marsh fungi in the Pacific Ocean with 165 taxa reported, while in the Indian Ocean, India reported the highest taxa (16 taxa). Many salt marsh areas remain unexplored, especially those habitats in the Indian and Pacific Oceans areas that are hotspots of biodiversity and novel fungal taxa based on the exploration of various habitats.
\end{abstract}

Keywords: halophytes; marine fungi; marine mycology; salt marsh fungi; worldwide distribution

\section{Introduction}

Salt marsh ecosystems are known for their high productivity, exceeding primary production estimates of species rich ecosystems (e.g., tropical rainforests, coral reefs) [1]. The flora in salt marsh ecosystems is mainly composed of grasses, herbs, and shrubs and these are terrestrial organisms variously adapted to, or tolerant of, a semi-marine environment. Halophytes are a diverse group of plants that have a worldwide distribution, and grow in different climatic regions, wherein soils have high salinity levels [2]. Halophytes are common in temperate and Mediterranean climates, and fewer both in the tropics and at high latitudes [3-6]. The vegetation in these ecosystems shows the vertical zonation of different communities as tidal submergence decreases with increasing elevation, and species tolerance to changing gradient conditions. Salt marsh vegetation generally increases the attenuation of both tidal currents and waves as they pass over the vegetated area and immobilize elements with their sediments. Furthermore, halophytes serve as a natural buffer, protecting other shoreline ecosystems from human impacts and disturbances. The 
area provides a habitat and nursery for marine organisms [7]. Worldwide, salt marshes cover an area of 5,495,089 hectare in 43 countries [8].

There are over 500 species of salt marsh plants worldwide [9]. The families Amaranthaceae (subfamilies Chenopodiaceae, Salicornieae), Poaceae, Juncaceae, and Cyperaceae are the major vegetation in salt marsh ecosystems, while the minor components are Plumbaginaceae and Frankeniaceae [3], and are represented in Figures 1 and 2. Salinity, latitude, region of the world, the frequency and duration of tidal flooding, substrate, oxygen and nutrient availability, surface elevation, competition among species, disturbance by wrack deposition are interacting factors that influence the species of halophytes in the salt marshes $[10,11]$. For example, Spartina alterniflora is a dominant grass from mid-tide to high-tide levels in temperate Eastern North America, while Puccinellia dominates in boreal and arctic marshes $[10,11]$.
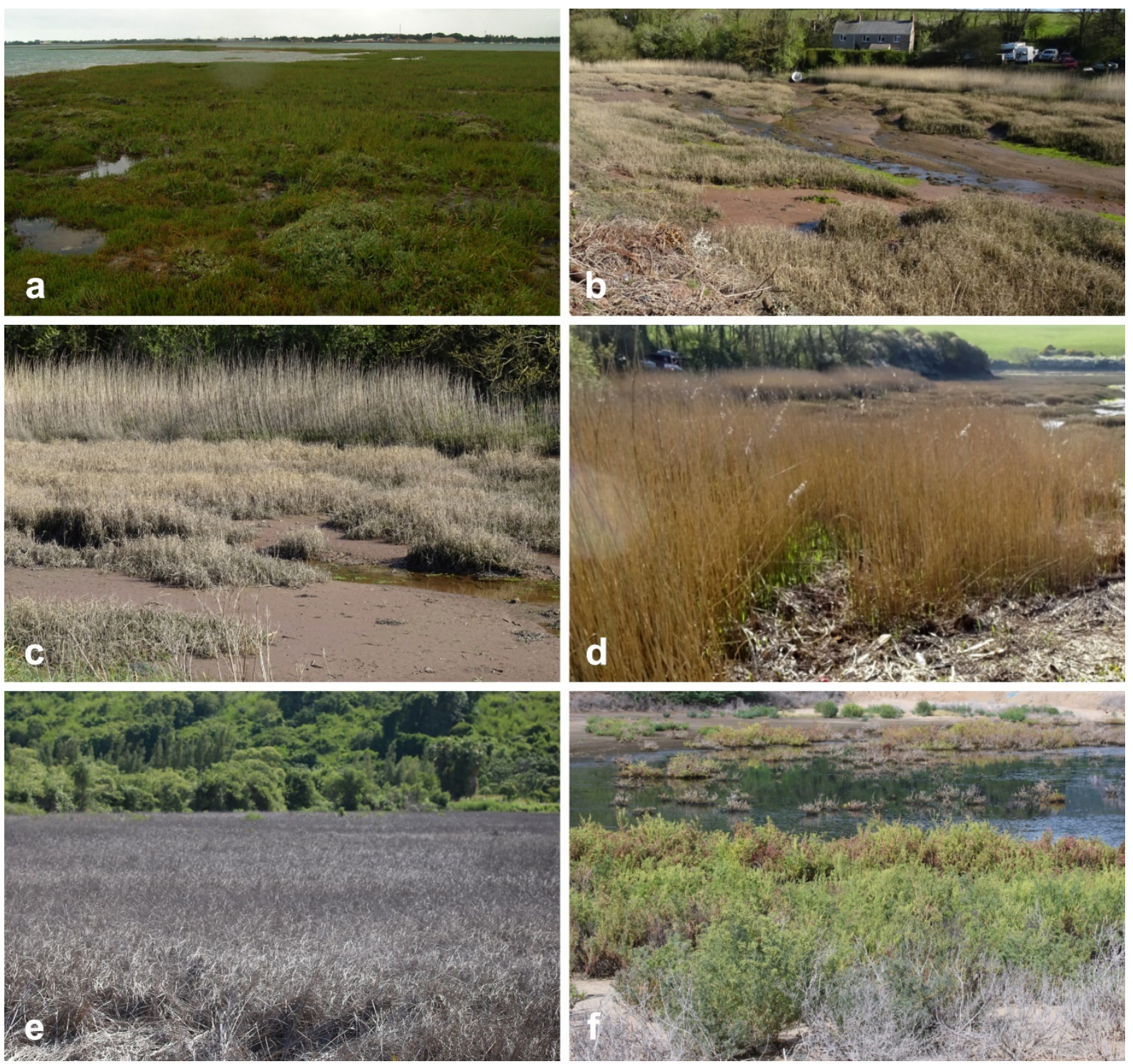

Figure 1. Salt marsh ecosystems in UK (a-d) and Thailand (e-f). (b-d) Tidal grasses, Spartina townsendii (Poaceae) and Phragmites (Poaceae), dominate the salt marsh in UK (50 49 $\left.55.4^{\prime \prime} \mathrm{N} 0^{\circ} 58^{\prime} 25.1^{\prime \prime} \mathrm{W} ; 51^{\circ} 43^{\prime} 03.1^{\prime \prime} \mathrm{N} 5^{\circ} 10^{\prime} 24.8^{\prime \prime} \mathrm{W}\right)$; (e) Spartina (Poaceae) (12 $\left.22^{\prime} 4.0^{\prime \prime} \mathrm{N} 99^{\circ} 59^{\prime} 6.7^{\prime \prime} \mathrm{E}\right)$ (f) and Suaeda (Amaranthaceae) $\left(12^{\circ} 10^{\prime} 19.6^{\prime \prime} \mathrm{N} 99^{\circ} 58^{\prime} 20.3^{\prime \prime} \mathrm{E}\right)$ in tidal marsh areas in southern Thailand. 

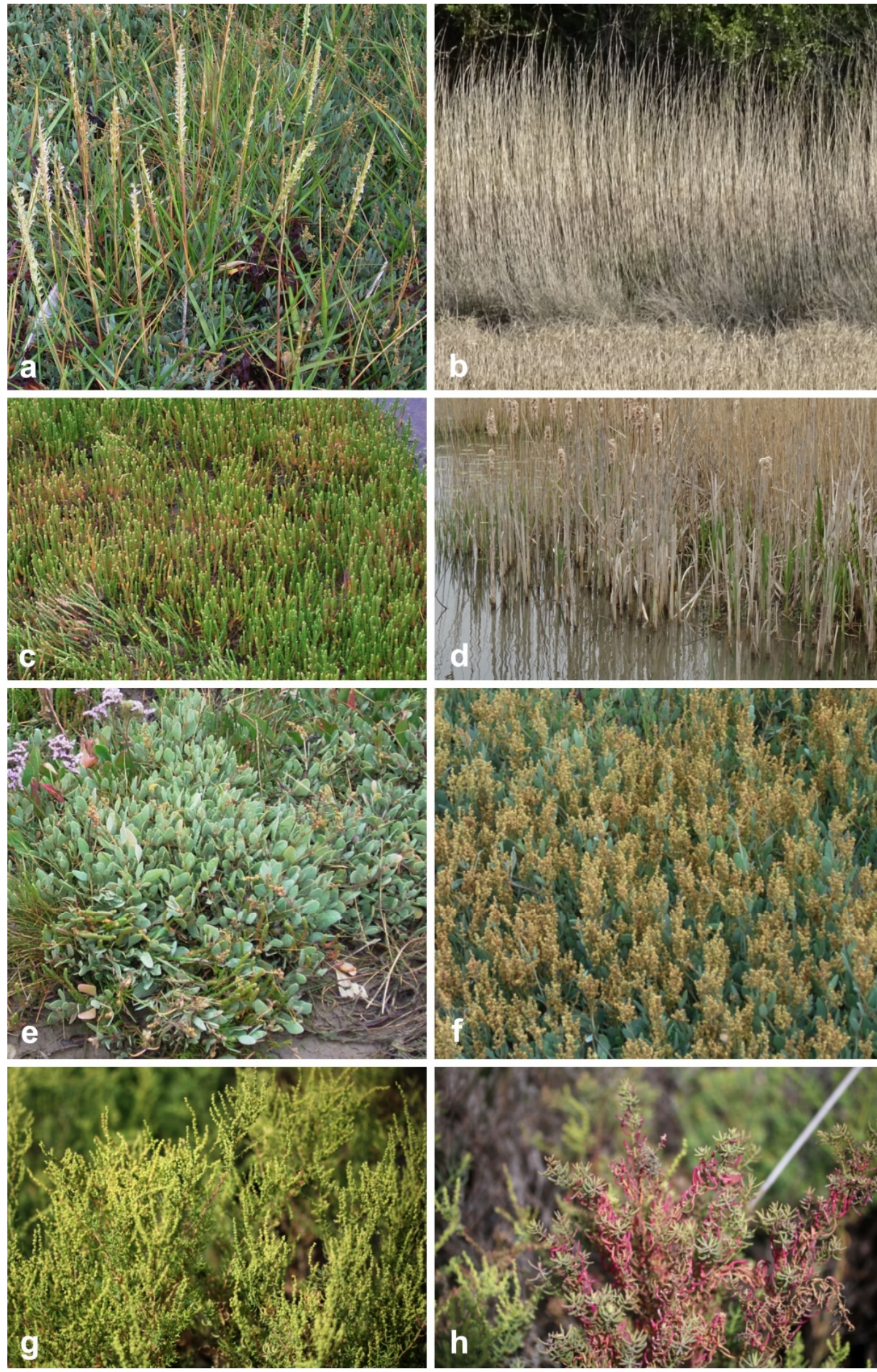

Figure 2. Halophytes in salt marsh ecosystems: (a) flowering inflorescence of Spartina, (b) Phragmites, (c) Salicornia, (d) Typha, (e,f) Atriplex, and (g,h) Suaeda. 
Major studies on halophytes focus on ecology and conservation [12-14]. One of these is the decomposition of vascular plant material wherein the detritus breakdown was reviewed in Pomeroy and Wiegert [15], Howarth and Hobbie [16], and Long and Mason [17]. The active decomposition processes in salt marsh ecosystems reflects to the relatively high rates of primary production. Three phases of plant decomposition were noted by Valiela et al. [18]. The early phase involves the leaching of soluble compounds, resulting in a fast rate of weight loss lasting for less than a month. Organic matter breakdown by microorganisms and continuous leaching of decayed products occurs in the second phase that lasts for a year. The last phase lasts for another year when there is a slow decay of refractory materials such as humates and fulvates [19].

The continuous breakdown of detritus into smaller fragments increases the surface-tovolume ratio and this is exposed to further microbial degradation. Bacteria and fungi are key decomposers in the salt marsh ecosystem that are essential for the transformation and recycling of nutrients through the environment. The colonization of fungi on standing dead halophytes commences during the early stages of decomposition before leaf fall to the salt marsh sediment surface $[20,21]$. The decomposition of the senescent tissues of halophytes by salt marsh fungi is brought about by the direct penetration of the host cell wall and the production of enzymes active in degrading lignocellulosic compounds, such as lignin, cellulose, and hemicellulose [22-26]. Bacterial communities are the major decomposers in the latter stage of decomposition $[27,28]$. Studies in salt marsh ecosystems not only consider microbial activity and the recycling of nutrients, but also bacterial [29,30] and fungal diversity [20,31,32].

The present review compiles the published data of fungi from halophytes, including their geographical distribution and host association. When compared to other fungal groups, salt marsh fungi are underexplored, and this review brings together the research efforts on these undiscovered habitats and plants. The pertinent literature from bibliographic databases (e.g., Scopus, Web of Science, Google Scholar) and published resources on salt marsh fungi documenting halophytes were compiled. Published works, wherein the documented fungal taxa were observed directly from halophytic substrates, are included (Table 1). The different host parts, living and dead, that are either partly or wholly submerged are documented, as well as drift plant portions washed up in salt marsh areas. Salt marsh fungi isolated using cultivation-dependent techniques were not included since it is not known if these fungi were actively growing and reproducing on the halophytes. The taxa were listed based on the recent outline of fungi and fungus-like taxa by Wijayawardene et al. [33]. Since previous works only listed the taxa and the hosts [34-36], here we include the plant parts where the fungus was observed, the location (country: state/province) where the host was collected, the life mode of the fungus, and the pertinent literature citations are included (Table 1). The accepted name of the host was based on the webpage of the World Flora Online consortium (http:/ / www.worldfloraonline.org/; accessed on 10 May 2021), GrassBase (https:/ / www.kew.org/data/grasses-db/sppindex.htm; accessed on 10 May 2021) and CRC World Dictionary of Grasses by Quattrocchi [37]. The graphs presented in the next sections summarizes the information from Table 1 and was developed using data visualization tools (Excel Office 365, Tableau Desktop Professional Edition 19.2.2). 
Table 1. Geographical distribution of salt marsh fungi recorded from various halophytes.

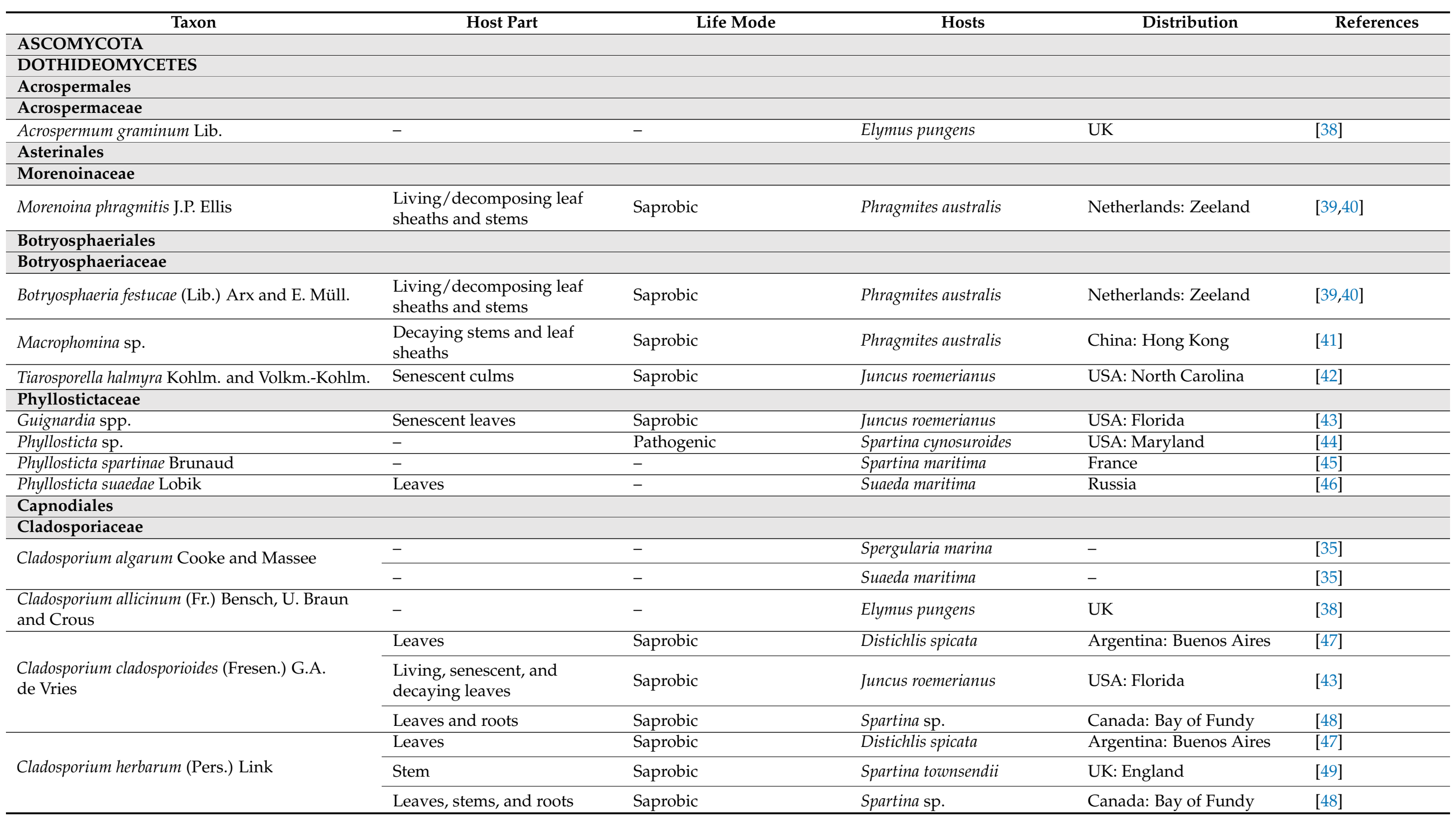


Table 1. Cont.

\begin{tabular}{|c|c|c|c|c|c|}
\hline Taxon & Host Part & Life Mode & Hosts & Distribution & References \\
\hline Cladosporium macrocarpum Preuss & Leaves & Saprobic & Spartina sp. & Canada: Bay of Fundy & {$[48]$} \\
\hline \multirow[t]{4}{*}{ Cladosporium sphaerospermum Penz. } & $\begin{array}{l}\text { Living, senescent, and } \\
\text { decaying leaves }\end{array}$ & Saprobic & Juncus roemerianus & USA: Florida & {$[43]$} \\
\hline & $\begin{array}{l}\text { Living/decomposing leaf } \\
\text { sheaths and blades }\end{array}$ & Saprobic & Phragmites australis & Netherlands: Zeeland & {$[39,41,50]$} \\
\hline & - & Saprobic & Spartina patens & USA: Rhode Island & [36] \\
\hline & - & Saprobic & Spartina sp. & Canada & [36] \\
\hline $\begin{array}{l}\text { Mucomycosphaerella eurypotami (Kohlm., } \\
\text { Volkm.-Kohlm. and O.E. Erikss.) Quaedvl. } \\
\text { and Crous }\end{array}$ & Senescent leaves & Saprobic & Juncus roemerianus & USA: North Carolina & {$[51]$} \\
\hline \multicolumn{6}{|l|}{ Mycosphaerellaceae } \\
\hline Fulvia fulva (Cooke) Cif. & Leaves and stems & Saprobic & Spartina sp. & Canada: Bay of Fundy & {$[48]$} \\
\hline \multirow{3}{*}{ Micronectriella agropyri Apinis and Chesters } & - & - & Elymus pungens & UK & [38] \\
\hline & - & - & Puccinellia maritima & UK & {$[38]$} \\
\hline & - & - & Spartina townsedii & UK & {$[38]$} \\
\hline $\begin{array}{l}\text { Mycosphaerella lineolata (Roberge ex Desm.) } \\
\text { J. Schröt. }\end{array}$ & - & - & Elymus pungens & UK & {$[38]$} \\
\hline \multirow{12}{*}{ Mycosphaerella salicorniae (Auersw.) Lindau } & - & - & Arthrocnemum subterminale & - & {$[35]$} \\
\hline & - & - & Limonium sp. & - & [35] \\
\hline & - & - & Sarcocornia perennis & - & [35] \\
\hline & - & - & Salicornia fruticosa & - & [35] \\
\hline & - & - & Salicornia procumbens & - & [35] \\
\hline & - & - & Salicornia europaea & - & {$[35]$} \\
\hline & - & - & Salicornia perennis & - & [35] \\
\hline & - & - & Sarcocornia fruticosa & - & [35] \\
\hline & $\begin{array}{l}\text { Drying stalks and } \\
\text { inflorescence }\end{array}$ & Saprobic & Salicornia sp. & India & {$[52]$} \\
\hline & Dried inflorescences & Saprobic & Salicornia virginica & Bermuda & {$[35,53]$} \\
\hline & - & Saprobic & Spartina marítima & Portugal: Alentejo, Lisbon & {$[54]$} \\
\hline & - & - & Suaeda vermiculata & - & [35] \\
\hline
\end{tabular}


Table 1. Cont.

\begin{tabular}{|c|c|c|c|c|c|}
\hline Taxon & Host Part & Life Mode & Hosts & Distribution & References \\
\hline & $\begin{array}{l}\text { Drying stalks and } \\
\text { inflorescence }\end{array}$ & Saprobic & Suaeda sp. & India & {$[52]$} \\
\hline \multirow{7}{*}{ Mycosphaerella spp. } & - & - & Elymus pungens & UK & {$[38]$} \\
\hline & $\begin{array}{l}\text { Decaying leaves, leaf } \\
\text { blades }\end{array}$ & Saprobic & Spartina alterniflora & $\begin{array}{l}\text { Argentina: Buenos Aires; } \\
\text { USA: Alabama, } \\
\text { California, Georgia, } \\
\text { Mississippi }\end{array}$ & {$[25,35,36,55-58]$} \\
\hline & - & - & Spartina cf. densiflora & USA: California & {$[25,35]$} \\
\hline & - & - & Spartina cf. pectinata & - & [35] \\
\hline & - & - & Spartina sp. & $\begin{array}{l}\text { Argentina: Buenos Aires; } \\
\text { Canada }\end{array}$ & {$[35,36]$} \\
\hline & Decaying leaf blades & Saprobic & Spartina foliosa & USA: California & [25] \\
\hline & $\begin{array}{l}\text { Leaf sheaths and blades, } \\
\text { stem }\end{array}$ & Saprobic & Spartina marítima & $\begin{array}{l}\text { Portugal: Alentejo, } \\
\text { Lisbon, Centro }\end{array}$ & {$[54,59]$} \\
\hline Mycosphaerella staticicola (Pat.) Dias & - & - & Armeria pungens & - & [35] \\
\hline $\begin{array}{l}\text { Rivilata ius Kohlm., Volkm.-Kohlm. and O.E. } \\
\text { Erikss. }\end{array}$ & $\begin{array}{l}\text { Tips of senescent, very old, } \\
\text { and brittle leaves }\end{array}$ & Saprobic & Juncus roemerianus & USA: North Carolina & {$[60]$} \\
\hline \multirow{3}{*}{ Septoria spp. } & $\begin{array}{l}\text { Living, senescent, and } \\
\text { decaying leaves }\end{array}$ & Saprobic & Juncus roemerianus & USA: Florida & [43] \\
\hline & $\begin{array}{l}\text { Living/decomposing leaf } \\
\text { sheaths }\end{array}$ & Saprobic & Phragmites australis & Netherlands: Zeeland & [39] \\
\hline & $\begin{array}{l}\text { Upper leaves, inflorescence, } \\
\text { seeds }\end{array}$ & Saprobic & Spartina alterniflora & USA: Rhode Island & [61] \\
\hline Septoria suaedae-australis Hansf. & Dead stems & Saprobic & Suaeda australis & South Australia & [62] \\
\hline \multirow[t]{2}{*}{ Sphaerulina albispiculata Tubaki } & Sheath & Saprobic & Spartina marítima & $\begin{array}{l}\text { Portugal: Alentejo, } \\
\text { Lisbon }\end{array}$ & {$[54]$} \\
\hline & Stem & Saprobic & Spartina marítima & Portugal: Alentejo & [63] \\
\hline \multirow[t]{2}{*}{ Sphaerulina orae-maris Linder } & - & - & Ammophila arenaria & - & {$[35]$} \\
\hline & Rhizome and root & Saprobic & Spartina densiflora & Argentina: Buenos Aires & {$[64]$} \\
\hline
\end{tabular}


Table 1. Cont.

\begin{tabular}{|c|c|c|c|c|c|}
\hline Taxon & Host Part & Life Mode & Hosts & Distribution & References \\
\hline & $\begin{array}{l}\text { Leaf sheaths and blades, } \\
\text { stem }\end{array}$ & Saprobic & Spartina marítima & $\begin{array}{l}\text { Portugal: Alentejo, } \\
\text { Lisbon, Algarve, Centro }\end{array}$ & {$[31,54,59,63]$} \\
\hline \multirow[t]{2}{*}{ Sphaerulina pedicellata T.W. Johnson } & - & Saprobic & Spartina townsendii & $x_{1}=0$ & {$[65]$} \\
\hline & Attached culms, stems & Saprobic, parasitic & Spartina alterniflora & USA: Rhode Island & {$[20,61]$} \\
\hline Sphaerulina sp. & $\begin{array}{l}\text { Senescent and decaying } \\
\text { leaves }\end{array}$ & Saprobic & Juncus roemerianus & USA: Florida & {$[43]$} \\
\hline \multicolumn{6}{|l|}{ Dothideales } \\
\hline \multicolumn{6}{|l|}{ Saccotheciaceae } \\
\hline Aureobasidium sp. & $\begin{array}{l}\text { Living, senescent, and } \\
\text { decaying leaves }\end{array}$ & Saprobic & Juncus roemerianus & USA: Florida & {$[43]$} \\
\hline Pseudoseptoria donacis (Pass.) B. Sutton & $\begin{array}{l}\text { Living/decomposing leaf } \\
\text { blades and sheaths }\end{array}$ & Saprobic & Phragmites australis & Netherlands: Zeeland & {$[39,50]$} \\
\hline Selenophoma sp. & $\begin{array}{l}\text { Senescent and decaying } \\
\text { leaves }\end{array}$ & Saprobic & Juncus roemerianus & USA: Florida & {$[43]$} \\
\hline \multicolumn{6}{|l|}{ Dothideaceae } \\
\hline $\begin{array}{l}\text { Scirrhia annulata Kohlm., Volkm.-Kohlm. and } \\
\text { O.E. Erikss. }\end{array}$ & Senescent culms and leaves & Saprobic & Juncus roemerianus & USA: North Carolina & {$[66]$} \\
\hline \multicolumn{6}{|l|}{ Dothideomycetes families incertae sedis } \\
\hline \multicolumn{6}{|l|}{ Eriomycetaceae } \\
\hline $\begin{array}{l}\text { Pseudorobillarda phragmitis (Cunnell) M. } \\
\text { Morelet }\end{array}$ & $\begin{array}{l}\text { Decaying stems and leaf } \\
\text { sheaths }\end{array}$ & Saprobic & Phragmites australis & China: Hong Kong & {$[41,67]$} \\
\hline Pseudorobillarda sp. & Dead stems & Saprobic & Spartina alterniflora & Canada & [36] \\
\hline \multicolumn{6}{|l|}{ Dothideomycetes genera incertae sedis } \\
\hline \multirow{3}{*}{$\begin{array}{l}\text { Bactrodesmium atrum M.B. Ellis } \\
\text { Lautitia danica (Berl.) S. Schatz }\end{array}$} & Living/decomposing stems & Saprobic & Phragmites australis & Netherlands: Zeeland & {$[40]$} \\
\hline & - & - & Elymus pungens & UK & [38] \\
\hline & - & - & Puccinellia maritima & UK & [38] \\
\hline Monodictys austrina Tubaki & Senescent leaves & Saprobic & Juncus roemerianus & USA: Florida & [43] \\
\hline Monodictys castaneae (Wallr.) S. Hughes & Leaves & Saprobic & Spartina sp. & Canada: Bay of Fundy & {$[48]$} \\
\hline $\begin{array}{l}\text { Neottiosporina australiensis B. Sutton and } \\
\text { Alcorn }\end{array}$ & $\begin{array}{l}\text { Living/decomposing leaf } \\
\text { blades and sheaths, stems }\end{array}$ & Saprobic & Phragmites australis & Netherlands: Zeeland & {$[39,40,50]$} \\
\hline Neottiosporina sp. & $\begin{array}{l}\text { Decaying stems and leaf } \\
\text { sheaths }\end{array}$ & Saprobic & Phragmites australis & China: Hong Kong & [41] \\
\hline
\end{tabular}


Table 1. Cont.

\begin{tabular}{|c|c|c|c|c|c|}
\hline Taxon & Host Part & Life Mode & Hosts & Distribution & References \\
\hline Otthia sp. & Senescent leaves & Saprobic & Juncus roemerianus & USA: Florida & [43] \\
\hline $\begin{array}{l}\text { Trichometasphaeria setulosa. (Sacc. and Roum.) } \\
\text { Apinis and Chesters ined. }\end{array}$ & - & - & Elymus pungens & UK & {$[38]$} \\
\hline Trichometasphaeria sp. & - & - & Elymus pungens & UK & [38] \\
\hline \multicolumn{6}{|l|}{ Microthyriales } \\
\hline \multicolumn{6}{|l|}{ Microthyriaceae } \\
\hline Microthyrium microscopicum Desm. & - & - & Spartina patens & - & [68] \\
\hline $\begin{array}{l}\text { Microthyrium gramineum Sacc., E. Bommer } \\
\text { and M. Rousseau }\end{array}$ & - & - & Elymus pungens & UK & {$[38]$} \\
\hline \multicolumn{6}{|l|}{ Muyocopronales } \\
\hline \multicolumn{6}{|l|}{ Muyocopronaceae } \\
\hline $\begin{array}{l}\text { Ellisiodothis inquinans (Ellis and Everh.) } \\
\text { Theiss. }\end{array}$ & - & Saprobic & Spartina alterniflora & Argentina: Buenos Aires & {$[36]$} \\
\hline \multicolumn{6}{|l|}{ Mytilinidiales } \\
\hline \multicolumn{6}{|l|}{ Mytilinidiaceae } \\
\hline Septonema secedens Corda & $\begin{array}{l}\text { Living, senescent, and } \\
\text { decaying leaves }\end{array}$ & Saprobic & Juncus roemerianus & USA: Florida & {$[43]$} \\
\hline \multicolumn{6}{|l|}{ Phaeotrichales } \\
\hline \multicolumn{6}{|l|}{ Phaeotrichaceae } \\
\hline \multirow{3}{*}{$\begin{array}{l}\text { Trichodelitschia bisporula (P. Crouan and H. } \\
\text { Crouan) E. Müll. and Arx } \\
\text { Pleosporales }\end{array}$} & - & - & Elymus pungens & UK & {$[38]$} \\
\hline & & & Spartina townsendii & UK & {$[38]$} \\
\hline & & & & & \\
\hline \multicolumn{6}{|l|}{ Amniculicolaceae } \\
\hline \multirow{4}{*}{$\begin{array}{l}\text { Neomassariosphaeria typhicola (P. Karst.) } \\
\text { Y. Zhang ter, J. Fourn. and K.D. Hyde }\end{array}$} & - & - & Juncus roemerianus & - & {$[35]$} \\
\hline & Decaying herbaceous stems & Saprobic & Spartina densiflora & Argentina: Buenos Aires & {$[64]$} \\
\hline & - & Saprobic & Spartina spp. & Argentina: Buenos Aires & {$[32,35,36]$} \\
\hline & - & Saprobic & $\begin{array}{l}\text { Unidentified saltmarsh } \\
\text { plants }\end{array}$ & USA: Mississippi & [58] \\
\hline \multicolumn{6}{|l|}{ Camarosporiaceae } \\
\hline Camarosporium feurichii Henn. & $\begin{array}{l}\text { Living/decomposing leaf } \\
\text { sheaths }\end{array}$ & Saprobic & Phragmites australis & Netherlands: Zeeland & [39] \\
\hline
\end{tabular}




\section{Taxon}

Camarosporium palliatum Kohlm. and E. Kohlm.

Camarosporium roumeguerei Sacc.

\begin{tabular}{ll} 
& Host Part \\
\hline- & - \\
\hline- & - \\
\hline- & $S$ \\
\hline- & - \\
\hline- & - \\
\hline- & - \\
\hline- & - \\
\hline Twigs & - \\
\hline- & - \\
\hline- & -
\end{tabular}

Life Mode

Saprobic or perthophytic

Hosts

Sarcocornia perennis

Salicornia sp.

Salicornia virginica

Salt marsh plants

Suaeda vermiculata

Atripex halimus

Atripex sp.

Distichlis spicata

Salicornia europaea

Sarcocornia fruticosa Salicornia sp.

$-$

Saprobic or perthophytic Salt marsh plants

Leaf sheaths and blades, stem

Saprobic

Spartina maritima

Spartina maritima

Suaeda maritima

Twigs

Camarosporium salicorniae Hansf.

Camarosporium spp.

Living/decomposing leaf

Sarcocornia quinqueflora

sheaths and stems

Camarosporium suaedae-fruticosae S. Ahmad

Dead branches

$-$

Coniothyriaceae

Coniothyrium obiones Jaap

$-$

Saprobic

Phragmites australis

Saprobic

Suaeda vermiculata

- $\quad$ Atriplex portulacoides

Salt marsh plants

Spartina maritima

Juncus roemerianus

Saprobic

Living, senescent, and

decaying leaves

Saprobic

Coniothyrium spp.

Cyclothyriellaceae 
Table 1. Cont.

\begin{tabular}{|c|c|c|c|c|c|}
\hline Taxon & Host Part & Life Mode & Hosts & Distribution & References \\
\hline $\begin{array}{l}\text { Massariosphaeria erucacea Kohlm., } \\
\text { Volkm.-Kohlm. and O.E. Erikss. }\end{array}$ & Senescent culms and leaves & Saprobic & Juncus roemerianus & USA: North Carolina & {$[66]$} \\
\hline $\begin{array}{l}\text { Massariosphaeria scirpina (G. Winter) } \\
\text { Leuchtm. }\end{array}$ & - & Saprobic & Spartina sp. & $\begin{array}{l}\text { USA: Florida, North } \\
\text { Carolina }\end{array}$ & [71] \\
\hline Massariosphaeria sp. & Living/decomposing stems & Saprobic & Phragmites australis & Netherlands: Zeeland & [40] \\
\hline \multicolumn{6}{|l|}{ Dictyosporiaceae } \\
\hline Dictyosporium oblongum (Fuckel) S. Hughes & $\begin{array}{l}\text { Living/decomposing leaf } \\
\text { blades and sheaths, stems }\end{array}$ & Saprobic & Phragmites australis & Netherlands: Zeeland & {$[39,40,50]$} \\
\hline \multirow{3}{*}{$\begin{array}{l}\text { Dictyosporium pelagicum (Linder) G.C. } \\
\text { Hughes ex E.B.G. Jones }\end{array}$} & Decomposing culms & Saprobic & Spartina alterniflora & USA: Rhode Island & {$[35,61]$} \\
\hline & - & - & Spartina spp. & - & [32] \\
\hline & $\begin{array}{l}\text { Leaf sheaths and blades, } \\
\text { stem }\end{array}$ & Saprobic & Spartina maritima & $\begin{array}{l}\text { Portugal: Alentejo, } \\
\text { Lisbon, Algarve, Centro }\end{array}$ & {$[54,59,63]$} \\
\hline $\begin{array}{l}\text { Jalapriya toruloides (Corda) M.J. D'souza, } \\
\text { Hong Y. Su, Z.L. Luo and K.D. Hyde }\end{array}$ & Stems & Saprobic & Spartina sp. & UK & [72] \\
\hline \multicolumn{6}{|l|}{ Didymellaceae } \\
\hline Ascochyta cf. arundinariae Tassi & $\begin{array}{l}\text { Living/decomposing leaf } \\
\text { blades and sheaths }\end{array}$ & Saprobic & Phragmites australis & Netherlands: Zeeland & {$[39,50]$} \\
\hline Ascochyta leptospora (Trail) Hara & $\begin{array}{l}\text { Living/decomposing leaf } \\
\text { sheaths }\end{array}$ & Saprobic & Phragmites australis & Netherlands: Zeeland & [39] \\
\hline Ascochyta salicorniae-patulae (Trotter) Melnik & - & Saprobic, parasitic & Salicornia spp. & $\begin{array}{l}\text { Canada, Denmark, } \\
\text { Germany, India, UK, USA }\end{array}$ & {$[52]$} \\
\hline \multirow[t]{2}{*}{ Ascochyta spp. } & $\begin{array}{l}\text { Living/decomposing leaf } \\
\text { sheaths }\end{array}$ & Saprobic & Phragmites australis & Netherlands: Zeeland & [39] \\
\hline & Sheath & Saprobic & Spartina marítima & Portugal: Alentejo & {$[54]$} \\
\hline Chaetasbolisia sp. & $\begin{array}{l}\text { Decaying stems and leaf } \\
\text { sheaths }\end{array}$ & Saprobic & Phragmites australis & China: Hong Kong & {$[41]$} \\
\hline Didymella glacialis Rehm & $\begin{array}{l}\text { Living/decomposing leaf } \\
\text { blades and sheaths, stems }\end{array}$ & Saprobic & Phragmites australis & Netherlands: Zeeland & {$[39,40,50]$} \\
\hline $\begin{array}{l}\text { Didymella glomerata (Corda) Qian Chen and } \\
\text { L. Cai }\end{array}$ & Rhizome and basal area & Saprobic & Spartina densiflora & Argentina: Buenos Aires & {$[64]$} \\
\hline \multirow[t]{2}{*}{ Didymella spp. } & $\begin{array}{l}\text { Living/decomposing leaf } \\
\text { blades and sheaths }\end{array}$ & Saprobic & Phragmites australis & Netherlands: Zeeland & {$[39,50]$} \\
\hline & - & Pathogenic & Spartina cynosuroides & USA: Louisiana & {$[44]$} \\
\hline
\end{tabular}


Table 1. Cont.

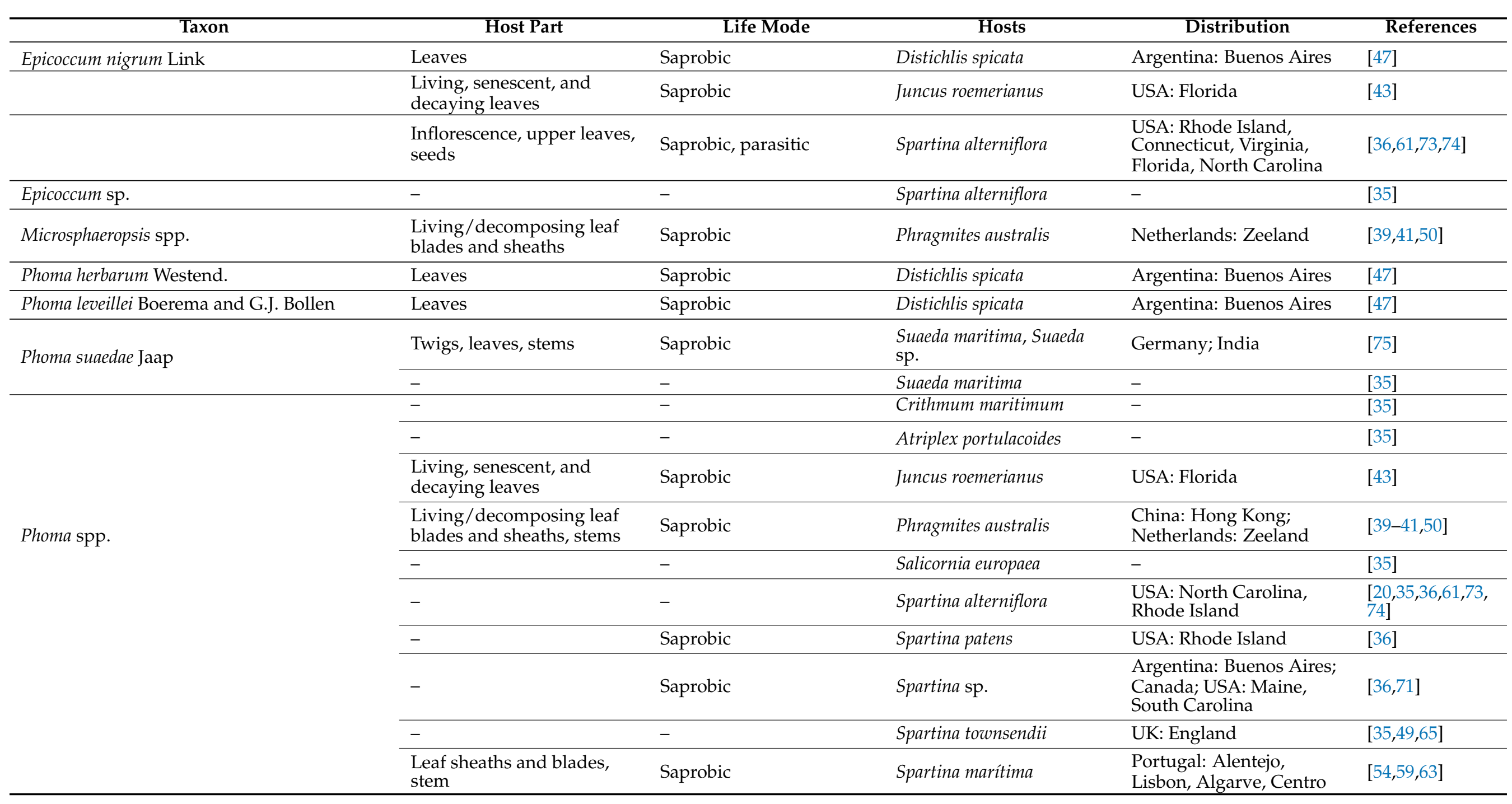


Table 1. Cont.

\begin{tabular}{|c|c|c|c|c|c|}
\hline Taxon & Host Part & Life Mode & Hosts & Distribution & References \\
\hline $\begin{array}{l}\text { Paraboeremia putaminum (Speg.) Qian Chen } \\
\text { and L. Cai }\end{array}$ & Leaves & Saprobic & Distichlis spicata & Argentina: Buenos Aires & [47] \\
\hline \multirow{2}{*}{ Stagonosporopsis salicorniae (Magnus) Died. } & - & - & Salicornia europaea & - & [35] \\
\hline & - & - & Salicornia patula & - & [35] \\
\hline \multicolumn{6}{|l|}{ Didymosphaeriaceae } \\
\hline \multirow{2}{*}{$\begin{array}{l}\text { Didymosphaeria lignomaris Strongman and } \\
\text { J.D. Mill. }\end{array}$} & Basal area of the sheath & Saprobic & Spartina densiflora & Argentina: Buenos Aires & {$[64]$} \\
\hline & - & - & Spartina spp. & - & [32] \\
\hline $\begin{array}{l}\text { Paraphaeosphaeria apicicola Kohlm., } \\
\text { Volkm.-Kohlm. and O.E. Erikss. }\end{array}$ & Senescent leaves & Saprobic & Juncus roemerianus & USA: North Carolina & [51] \\
\hline $\begin{array}{l}\text { Paraphaeosphaeria pilleata Kohlm., } \\
\text { Volkm.-Kohlm. and O.E. Erikss. }\end{array}$ & Senescent culms & Saprobic & Juncus roemerianus & USA: North Carolina & [77] \\
\hline \multirow{2}{*}{$\begin{array}{l}\text { Paraphaeosphaeria michotii (Westend.) O.E. } \\
\text { Erikss. }\end{array}$} & - & - & Elymus pungens & UK & {$[38]$} \\
\hline & $\begin{array}{l}\text { Living/decomposing leaf } \\
\text { sheaths }\end{array}$ & Saprobic & Phragmites australis & Netherlands: Zeeland & [39] \\
\hline $\begin{array}{l}\text { Pseudopithomyces atro-olivaceus (Cooke and } \\
\text { Harkn.) G. Guevara, K.C. Cunha and Gené }\end{array}$ & $\begin{array}{l}\text { Senescent and decaying } \\
\text { leaves }\end{array}$ & Saprobic & Juncus roemerianus & USA: Florida & [43] \\
\hline $\begin{array}{l}\text { Pseudopithomyces chartarum (Berk. and M.A. } \\
\text { Curtis) Jun F. Li, Ariyaw. and K.D. Hyde }\end{array}$ & Senescent leaves & Saprobic & Juncus roemerianus & USA: Florida & [43] \\
\hline \multirow{2}{*}{$\begin{array}{l}\text { Pseudopithomyces maydicus (Sacc.) Jun F. Li, } \\
\text { Ariyaw. and K.D. Hyde }\end{array}$} & $\begin{array}{l}\text { Senescent and decaying } \\
\text { leaves }\end{array}$ & Saprobic & Juncus roemerianus & USA: Florida & [43] \\
\hline & $\begin{array}{l}\text { Decaying stems and leaf } \\
\text { sheaths }\end{array}$ & Saprobic & Phragmites australis & China: Hong Kong & [41] \\
\hline \multirow[t]{2}{*}{$\begin{array}{l}\text { Spegazzinia tessarthra (Berk. and M.A. Curtis) } \\
\text { Sacc. }\end{array}$} & Living leaves & & Juncus roemerianus & USA: Florida & [43] \\
\hline & $\begin{array}{l}\text { Decaying stems and leaf } \\
\text { sheaths }\end{array}$ & Saprobic & Phragmites australis & China: Hong Kong & [41] \\
\hline \multirow{2}{*}{$\begin{array}{l}\text { Tremateia halophila Kohlm., Volkm.-Kohlm. } \\
\text { and O.E. Erikss. }\end{array}$} & $\begin{array}{l}\text { Lower and middle parts of } \\
\text { senescent culms }\end{array}$ & Saprobic & Juncus roemerianus & USA: North Carolina & [78] \\
\hline & - & Saprobic & Spartina maritima & $\begin{array}{l}\text { Portugal: Alentejo, } \\
\text { Lisbon }\end{array}$ & [54] \\
\hline
\end{tabular}


Table 1. Cont.

\begin{tabular}{|c|c|c|c|c|c|}
\hline Taxon & Host Part & Life Mode & Hosts & Distribution & References \\
\hline \multicolumn{6}{|l|}{ Lentitheciaceae } \\
\hline $\begin{array}{l}\text { Halobyssothecium estuariae B. Devadatha, } \\
\text { Calabon, K.D. Hyde and E.B.G. Jones }\end{array}$ & Dead culm & Saprobic & Phragmites australis & UK: Pembrokeshire & [79] \\
\hline \multirow{12}{*}{$\begin{array}{l}\text { Halobyssothecium obiones (P. Crouan and H. } \\
\text { Crouan) Dayarathne, E.B.G. Jones and } \\
\text { K.D. Hyde }\end{array}$} & $\begin{array}{l}\text { Drift stems, attached and } \\
\text { dead culms }\end{array}$ & Saprobic & Spartina alterniflora & $\begin{array}{l}\text { India: Maharashtra, Tamil } \\
\text { Nadu, Andhara Pradesh; } \\
\text { USA: Maine, Rhode } \\
\text { Island, Connecticut, } \\
\text { Massachusetts, New } \\
\text { Jersey, Maryland, } \\
\text { Virginia, North Carolina, } \\
\text { South Carolina, Florida, } \\
\text { Mississippi, Texas }\end{array}$ & $\begin{array}{l}{[20,35,52,61,71} \\
74,80-82]\end{array}$ \\
\hline & - & - & Spartina cynosuroides & - & [35] \\
\hline & Pod and rhizome & Saprobic & Spartina densiflora & Argentina: Buenos Aires & {$[64]$} \\
\hline & - & Saprobic & Spartina patens & USA: Rhode Island & [36] \\
\hline & Culms & Saprobic & Spartina sp. & UK: England, Hampshire & {$[79,83]$} \\
\hline & Stem & Saprobic & Spartina townsendii & UK: Hampshire, Wales & {$[49,65]$} \\
\hline & - & Saprobic & Spartina spp. & $\begin{array}{l}\text { USA: New Jersey, South } \\
\text { Carolina; Mississippi, } \\
\text { Argentina: Buenos Aires }\end{array}$ & {$[32,35,36,58,84]$} \\
\hline & $\begin{array}{l}\text { Stem, leaf sheaths, and } \\
\text { blades }\end{array}$ & Saprobic & Spartina marítima & $\begin{array}{l}\text { Portugal: Alentejo, } \\
\text { Lisbon, Algarve, Centro }\end{array}$ & {$[31,54,59,63]$} \\
\hline & - & Saprobic & $\begin{array}{l}\text { Unidentified saltmarsh } \\
\text { plants }\end{array}$ & USA: Mississippi & {$[55,58]$} \\
\hline & - & - & Elymus pungens & - & [35] \\
\hline & - & - & Atriplex portulacoides & - & [35] \\
\hline & - & - & Spartina townsendii & - & [35] \\
\hline $\begin{array}{l}\text { Halobyssothecium phragmitis M.S. Calabon, } \\
\text { E.B.G. Jones, S. Tibell and K.D. Hyde }\end{array}$ & Dead culm and stem & Saprobic & Phragmites sp. & Sweden: Gotland & [85] \\
\hline $\begin{array}{l}\text { Halobyssothecium versicolor M.S. Calabon, } \\
\text { E.B.G. Jones and K.D. Hyde }\end{array}$ & Dead stem & Saprobic & Atriplex portulacoides & UK: Hampshire & [85] \\
\hline
\end{tabular}


Table 1. Cont.

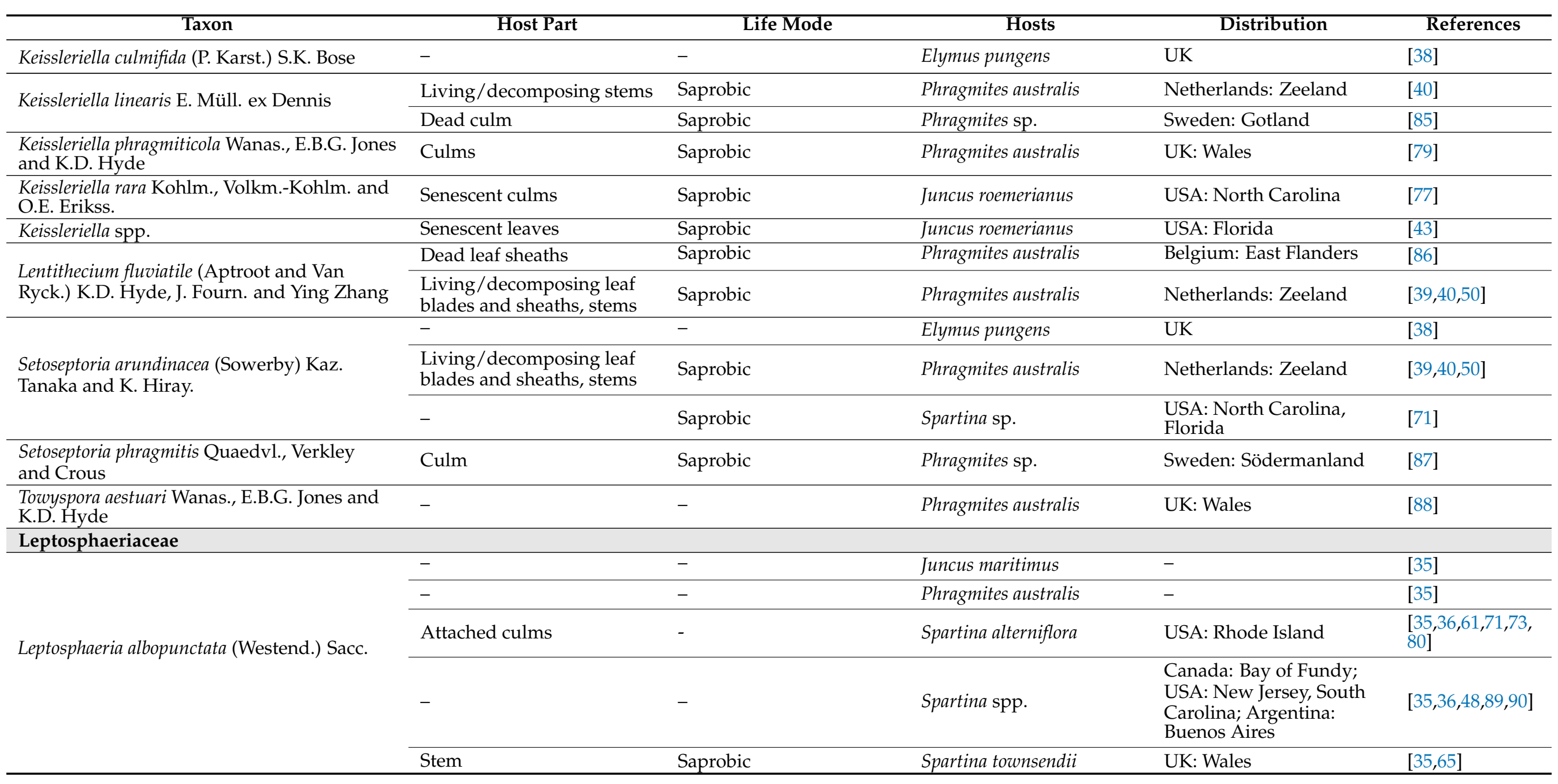


Table 1. Cont.

\begin{tabular}{|c|c|c|c|c|c|}
\hline Taxon & Host Part & Life Mode & Hosts & Distribution & References \\
\hline \multirow{3}{*}{$\begin{array}{l}\text { Leptosphaeria australiensis (Cribb and J.W. } \\
\mathrm{Cribb} \text { ) G.C. Hughes }\end{array}$} & $\begin{array}{l}\text { Senescent and decaying } \\
\text { leaves }\end{array}$ & Saprobic & Juncus roemerianus & USA: Florida & [43] \\
\hline & Pod & Saprobic & Spartina densiflora & Argentina: Buenos Aires & [64] \\
\hline & - & - & Spartina spp. & - & [32] \\
\hline $\begin{array}{l}\text { Leptosphaeria culmifraga (Fr.) Ces. and De } \\
\text { Not. }\end{array}$ & - & - & Elymus pungens & UK & [38] \\
\hline Leptosphaeria littoralis Sacc. & - & - & Elymus pungens & UK & [38] \\
\hline \multirow{4}{*}{ Leptosphaeria marina Ellis and Everh. } & - & - & Juncus roemerianus & & [35] \\
\hline & - & Saprobic & Spartina spp. & Canada; USA: New Jersey & $\begin{array}{l}{[32,35,36,65,89-} \\
91]\end{array}$ \\
\hline & - & - & Spartina townsendii & UK & {$[35,38]$} \\
\hline & $\begin{array}{l}\text { Leaf sheaths and blades, } \\
\text { stem }\end{array}$ & Saprobic & Spartina maritima & Portugal: Algarve & {$[31,59]$} \\
\hline \multirow{5}{*}{ Leptosphaeria orae-maris Linder } & - & Saprobic & Lysimachia maritima & USA: Massachusetts & {$[35,92]$} \\
\hline & - & Saprobic & Spartina alterniflora & $\begin{array}{l}\text { USA: Massachusetts, } \\
\text { Rhode Island, North } \\
\text { Carolina, Florida, Texas }\end{array}$ & {$[36,71,80,92]$} \\
\hline & Rhizome & Saprobic & Spartina densiflora & Argentina: Buenos Aires & [64] \\
\hline & - & - & Spartina spp. & - & [32] \\
\hline & - & Saprobic & Spartina townsendii & UK & {$[35,65,93]$} \\
\hline
\end{tabular}




\section{Taxon}

Taxon

Leptosphaeria pelagica E.B.G. Jones

Leptosphaeria peruvianae Speg.

Leptosphaeria spp.

Leptospharia peruoianaespeg.

\section{Life Mode}

Host Part

\begin{tabular}{ll}
- & - \\
\hline- & - \\
\hline $\begin{array}{l}\text { Decaying herbaceous } \\
\text { stems, dead culms, } \\
\text { decaying leaves }\end{array}$ & Sapr
\end{tabular}

$-$

Hosts

Elymus pungens

Puccinellia maritima

Saprobic

Spartina alterniflora

\begin{tabular}{lll}
\hline- & Saprobic & $S p a$ \\
\hline- & Saprobic & $S p a$ \\
\hline- & - & $S p a$ \\
\hline- & - & $S p a$
\end{tabular}

$-$

Sheath

Saprobic

Stem

Saprobic

Decaying stems

Saprobic

Decaying leaves

Saprobic

Decaying stems and leaf

sheaths

Saprobic

Spartina densiflora

Spartina patens

Spartina townsendii

Spartina spp.

-

Leaf sheaths and blades,

stem

- Spartina alterniflora

Saprobic

Saprobic

Spartina marítima

Spartina maritima

Sarcocornia perennis

Juncus roemerianus

Phragmites australis

Spartina maritima

Leptosphaeria suaedae Hansf.

Lindgomycetaceae

Arundellina typhae Wanas., E.B.G. Jones and

K.D. Hyde

Dead stem

Saprobic

Suaeda australis

Typha sp.

Phragmites australis

Elymus pungens

Living/decomposing stems Sapr

$-$
Living/decomposing stems Sap

robic

Lophiostoma sp.

(2)


Table 1. Cont.

\section{Taxon}

Host Part
Hosts

Distribution

References

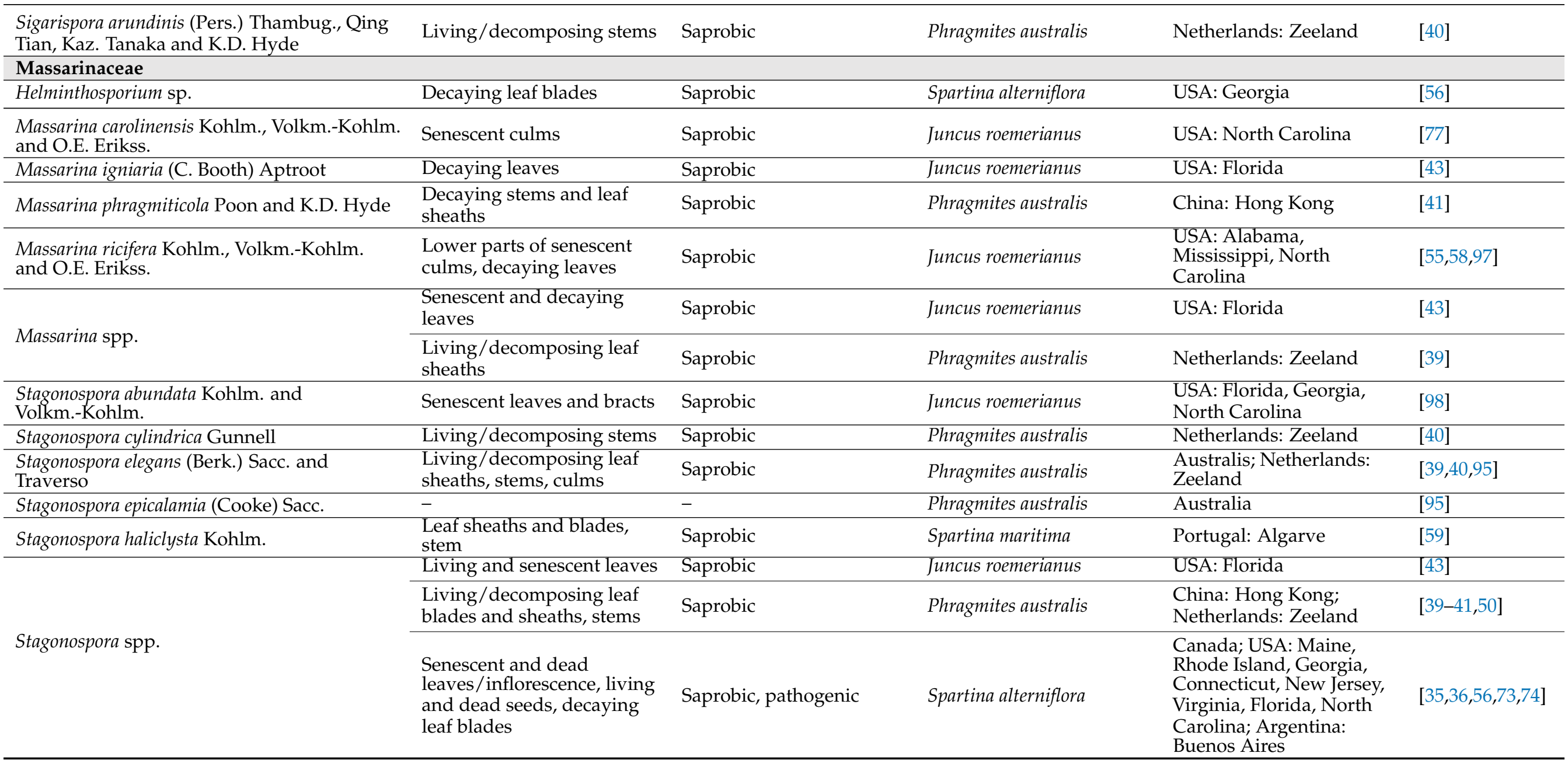


Table 1. Cont.

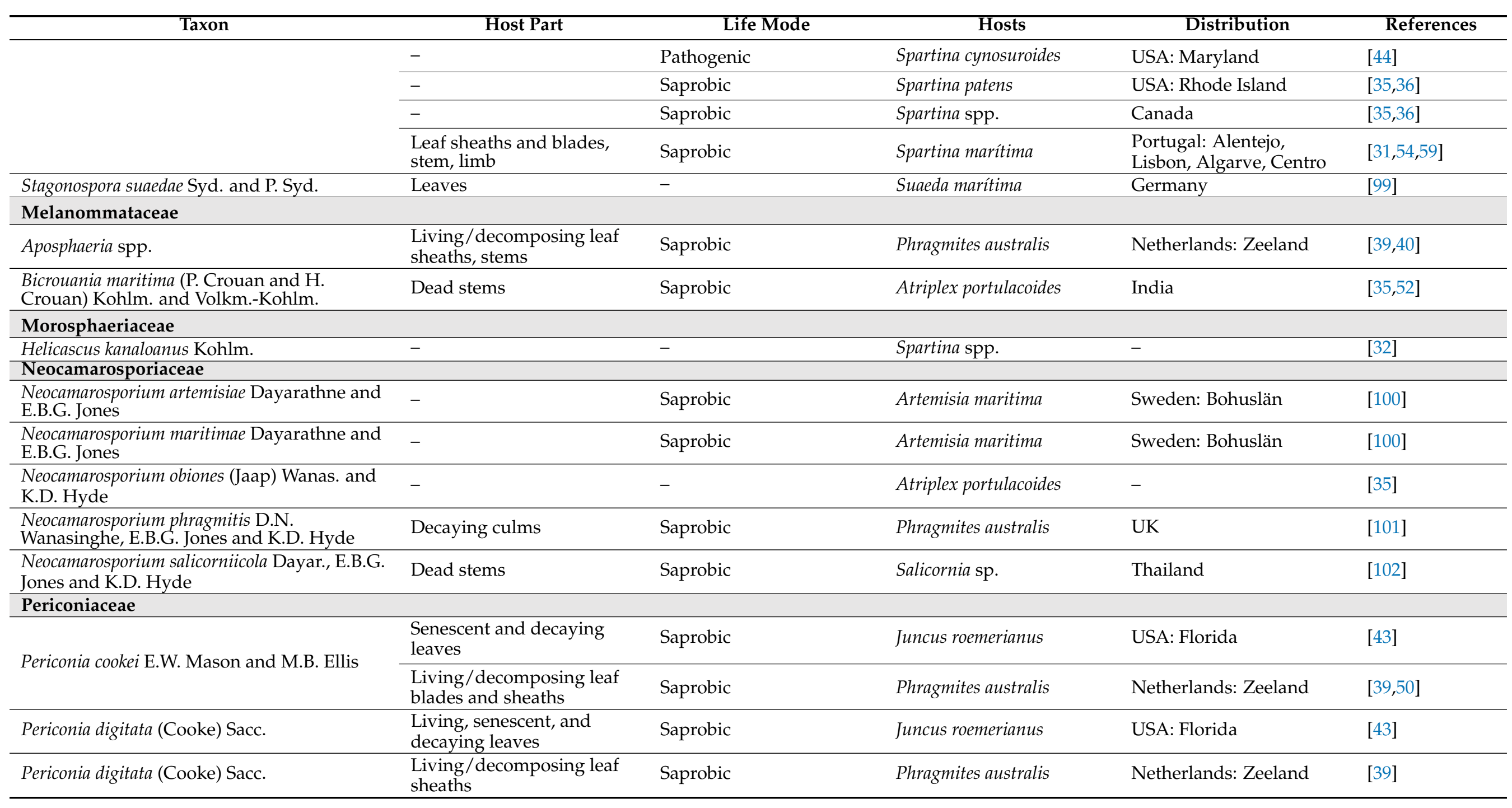


Table 1. Cont.

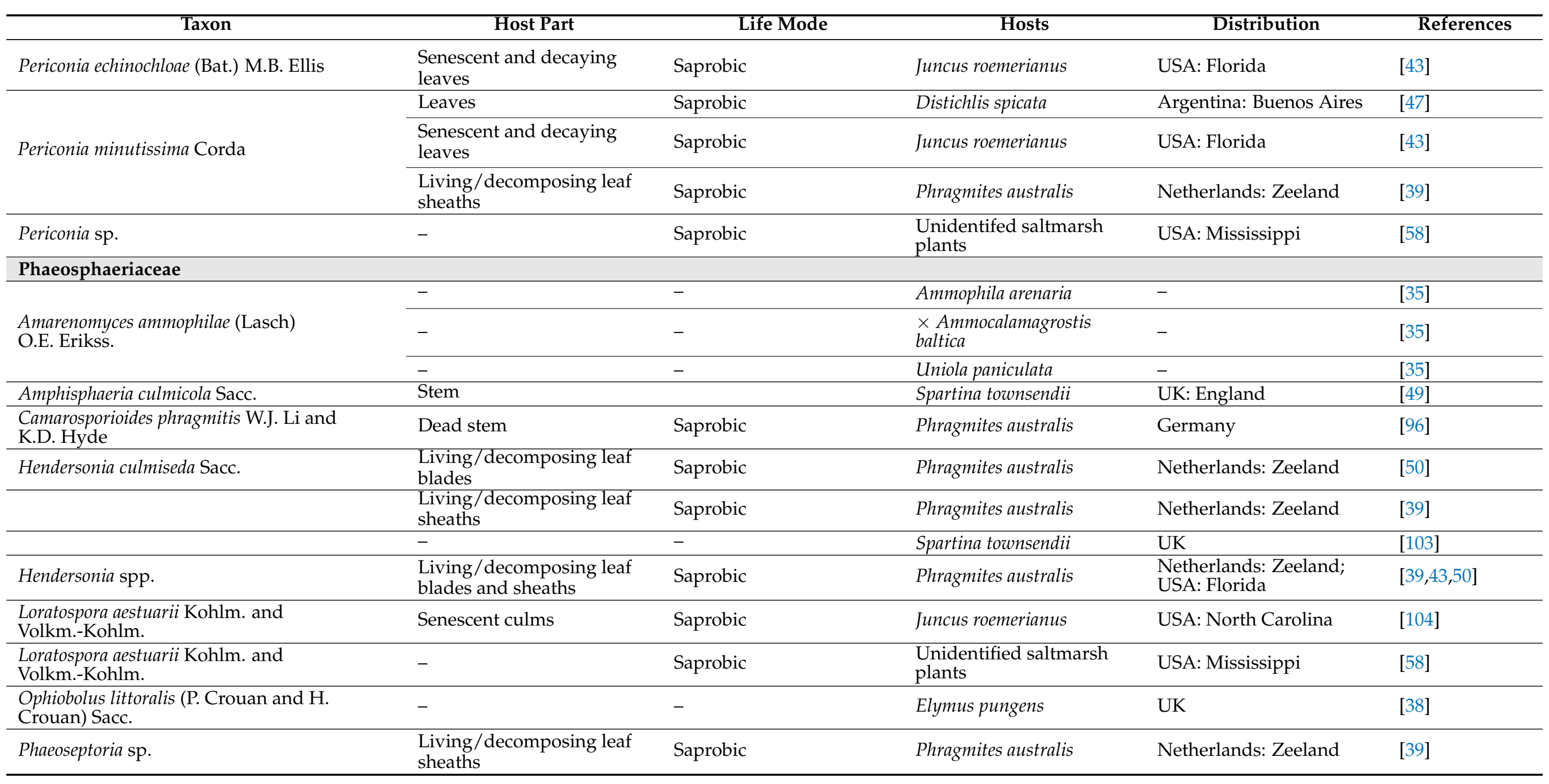


Table 1. Cont.

Taxon

Host Part

Life Mode

Phaeosphaeria anchiala Kohlm., Volkm.-Kohlm. and C.K.M. Tsui

Phaeosphaeria caricinella (P. Karst.) O.E

Erikss.

Phaeosphaeria culmorum (Auersw.) Leuchtm.

Phaeosphaeria eustoma (Fuckel) L. Holm

Phaeosphaeria fuckelii (Niessl) L. Holm

\begin{tabular}{l} 
Phaeosphaeria fuckelii (Niessl) L. Holm \\
\hline Phaeosphaeria gessneri Shoemaker and C.E.
\end{tabular}

Babc.

Phaeosphaeria halima (T.W. Johnson)

Shoemaker and C.E. Babc.

Phaeosphaeria herpotrichoides (De Not.) L

Holm

Phaeosphaeria juncina (Auersw.) L. Holm

Phaeosphaeria luctuosa (Niessl ex Sacc.) Y.

Otani and Mikawa
Senescent leaves

$-$

-

Saprobic

Living/decomposing leaf

blades and sheaths

Living/decomposing leaf

blades and sheaths, stems,

culms

$-$

$-$

$-$

Dead culms; Decaying leaves, leaf blades

- Spartina sp.

Saprobic

$-$

Saprobic

$-$

Saprobic

Decaying leaf blades

Saprobic

Saprobic

Saprobic

$-$

stem

-

$-$

Living/decomposing leaf

sheaths, stems

-
Saprobic

Saprobic

Elymus pungens

Hosts

Juncus roemerianus

Spartina sp.

Phragmites australis

Phragmites australis

Elymus pungens

Spartina spp.

Spartina alterniflora

Spartina densiflora

Spartina spp.

Spartina foliosa

Spartina maritima

Distribution

USA: Florida, Georgia

Maryland, North

Carolina, Virginia

USA: Florida, North Carolina

Netherlands: Zeeland

$[39,501$

Netherlands: Zeeland

UK

$-$

[38]

[32]

India: Kerala; USA:

California, Georgia, $\quad[25,35,52,55-$

Mississippi, Vancouver, $\quad 58,71,80]$

North Carolina

USA: California [25]

25]

[32]

USA: California

[25]

Portugal: Algarve, Centro [31]

Spartina patens

Juncus roemerianus

USA: North Carolina,

[71]

USA: Florida

[43]

Phragmites australis

Netherlands: Zeeland

$[39,40]$

Elymus pungens

UK

[38]

References

05]

1]

[38] 
Table 1. Cont.

\begin{tabular}{|c|c|c|c|c|c|}
\hline Taxon & Host Part & Life Mode & Hosts & Distribution & References \\
\hline \multirow{2}{*}{$\begin{array}{l}\text { Phaeosphaeria macrosporidium (E.B.G. Jones) } \\
\text { Shoemaker and C.E. Babc. }\end{array}$} & Decaying stems & Saprobic & Spartina $s p$ & UK: Wales, England & {$[65]$} \\
\hline & Stem & Saprobic & Spartina maritima & Portugal: Lisbon & {$[54,63]$} \\
\hline $\begin{array}{l}\text { Phaeosphaeria microscopica (P. Karst.) O.E. } \\
\text { Erikss. }\end{array}$ & - & - & Elymus pungens & UK & [38] \\
\hline \multirow{7}{*}{$\begin{array}{l}\text { Phaeosphaeria neomaritima (R.V. Gessner and } \\
\text { Kohlm.) Shoemaker and C.E. Babc. }\end{array}$} & - & - & Juncus maritimus & - & [35] \\
\hline & - & - & Juncus roemerianus & - & [35] \\
\hline & - & Saprobic & Juncus sp. & $\begin{array}{l}\text { Canada; India: } \\
\text { Maharashtra, Karnataka; } \\
\text { USA: Virginia, North } \\
\text { Carolina }\end{array}$ & {$[36,52,71,80]$} \\
\hline & - & - & Spartina alterniflora & - & [35] \\
\hline & - & Saprobic & Spartina spp. & $\begin{array}{l}\text { Canada; USA: North } \\
\text { Carolina, Virginia }\end{array}$ & {$[32,71,80]$} \\
\hline & - & - & Spartina townsendii & UK & {$[35,93]$} \\
\hline & Stem & Saprobic & Spartina marítima & Portugal: Alentejo & [63] \\
\hline $\begin{array}{l}\text { Phaeosphaeria nigrans (Roberge ex Desm.) L. } \\
\text { Holm }\end{array}$ & - & - & Elymus pungens & UK & [38] \\
\hline $\begin{array}{l}\text { Phaeosphaeria olivacea Kohlm., Volkm.-Kohlm. } \\
\text { and O.E. Erikss. }\end{array}$ & Senescent leaves & Saprobic & Juncus roemerianus & $\begin{array}{l}\text { USA: North Carolina, } \\
\text { Mississippi }\end{array}$ & {$[58,76]$} \\
\hline \multirow[b]{2}{*}{ Phaeosphaeria pontiformis (Fuckel) Leuchtm. } & - & - & Elymus pungens & UK & {$[38]$} \\
\hline & $\begin{array}{l}\text { Living/decomposing leaf } \\
\text { blades and sheaths, stems }\end{array}$ & Saprobic & Phragmites australis & Netherlands: Zeeland & {$[39,40,50]$} \\
\hline $\begin{array}{l}\text { Phaeosphaeria roemeriani Kohlm., } \\
\text { Volkm.-Kohlm. and O.E. Erikss. }\end{array}$ & $\begin{array}{l}\text { Senescent and decaying } \\
\text { leaves }\end{array}$ & Saprobic & Juncus roemerianus & $\begin{array}{l}\text { USA: Mississippi, North } \\
\text { Carolina }\end{array}$ & {$[55,58,60]$} \\
\hline \multirow{3}{*}{$\begin{array}{l}\text { Phaeosphaeria spartinae (Ellis and Everh.) } \\
\text { Shoemaker and C.E. Babc. }\end{array}$} & - & Saprobic & Spartina spp. & India: Kerala & {$[32,52]$} \\
\hline & $\begin{array}{l}\text { Decaying herbaceous stems } \\
\text { and pod }\end{array}$ & Saprobic & Spartina densiflora & Argentina: Buenos Aires & {$[64]$} \\
\hline & - & Saprobic & Spartina maritima & Portugal: Lisbon & {$[54]$} \\
\hline
\end{tabular}


Table 1. Cont.

\begin{tabular}{|c|c|c|c|c|c|}
\hline Taxon & Host Part & Life Mode & Hosts & Distribution & References \\
\hline \multirow{6}{*}{ Phaeosphaeria spartinicola Leuchtm. } & - & Saprobic & Juncus sp. & India & {$[52]$} \\
\hline & $\begin{array}{l}\text { Dead leaves, decaying leaf } \\
\text { blades }\end{array}$ & Saprobic & Spartina alterniflora & $\begin{array}{l}\text { Mexico; USA: Alabama, } \\
\text { California, Georgia, } \\
\text { Mississippi; Canada: } \\
\text { Nova Scotia, New } \\
\text { Brunswick }\end{array}$ & {$[25,36,55-58]$} \\
\hline & Pod, leaf blades & Saprobic & Spartina densiflora & $\begin{array}{l}\text { Argentina: Buenos Aires; } \\
\text { USA: California }\end{array}$ & {$[25,64]$} \\
\hline & - & - & Spartina spp. & - & [32] \\
\hline & Leaf blades & Saprobic & Spartina foliosa & USA: California & [25] \\
\hline & $\begin{array}{l}\text { Leaf sheaths and blades, } \\
\text { stem, limb }\end{array}$ & Saprobic & Spartina marítima & $\begin{array}{l}\text { Portugal: Alentejo, } \\
\text { Lisbon, Algarve, Centro }\end{array}$ & {$[31,54,59,63]$} \\
\hline \multirow[t]{2}{*}{ Phaeosphaeria spp. } & $\begin{array}{l}\text { Living/decomposing leaf } \\
\text { blades and sheaths, stems }\end{array}$ & Saprobic & Phragmites australis & Netherlands: Zeeland & {$[39,40,50]$} \\
\hline & - & Saprobic & Spartina alterniflora & USA: Rhode Island & {$[74]$} \\
\hline Sclerostagonospora sp. & $\begin{array}{l}\text { Decaying stems and leaf } \\
\text { sheaths }\end{array}$ & Saprobic & Phragmites australis & China: Hong Kong & [41] \\
\hline Septoriella spp. & $\begin{array}{l}\text { Decaying stems and leaf } \\
\text { sheaths and blades, stems }\end{array}$ & Saprobic & Phragmites australis & $\begin{array}{l}\text { China: Hong Kong; } \\
\text { Netherlands: Zeeland }\end{array}$ & {$[39-41,50]$} \\
\hline \multirow[t]{2}{*}{ Septoriella thalassica (Speg.) Nag Raj } & - & - & Distichlis spicata & - & [35] \\
\hline & & & Distichlis spicata & & [35] \\
\hline $\begin{array}{l}\text { Septoriella unigalerita Kohlm. and } \\
\text { Volkm.-Kohlm. }\end{array}$ & Senescent leaves & Saprobic & Juncus roemerianus & USA: North Carolina & {$[98]$} \\
\hline \multirow[t]{3}{*}{ Septoriella vagans (Niessl) Y. Marín and Crous } & - & - & Elymus pungens & UK & {$[38]$} \\
\hline & - & - & Puccinellia maritima & UK & [38] \\
\hline & - & Saprobic & Spartina alterniflora & USA: Rhode Island & {$[74]$} \\
\hline \multicolumn{6}{|l|}{ Pleomassariaceae } \\
\hline Splanchnonema sp. & $\begin{array}{l}\text { Living, senescent, and } \\
\text { decaying leaves }\end{array}$ & Saprobic & Juncus roemerianus & USA: Florida & [43] \\
\hline
\end{tabular}


Table 1. Cont.

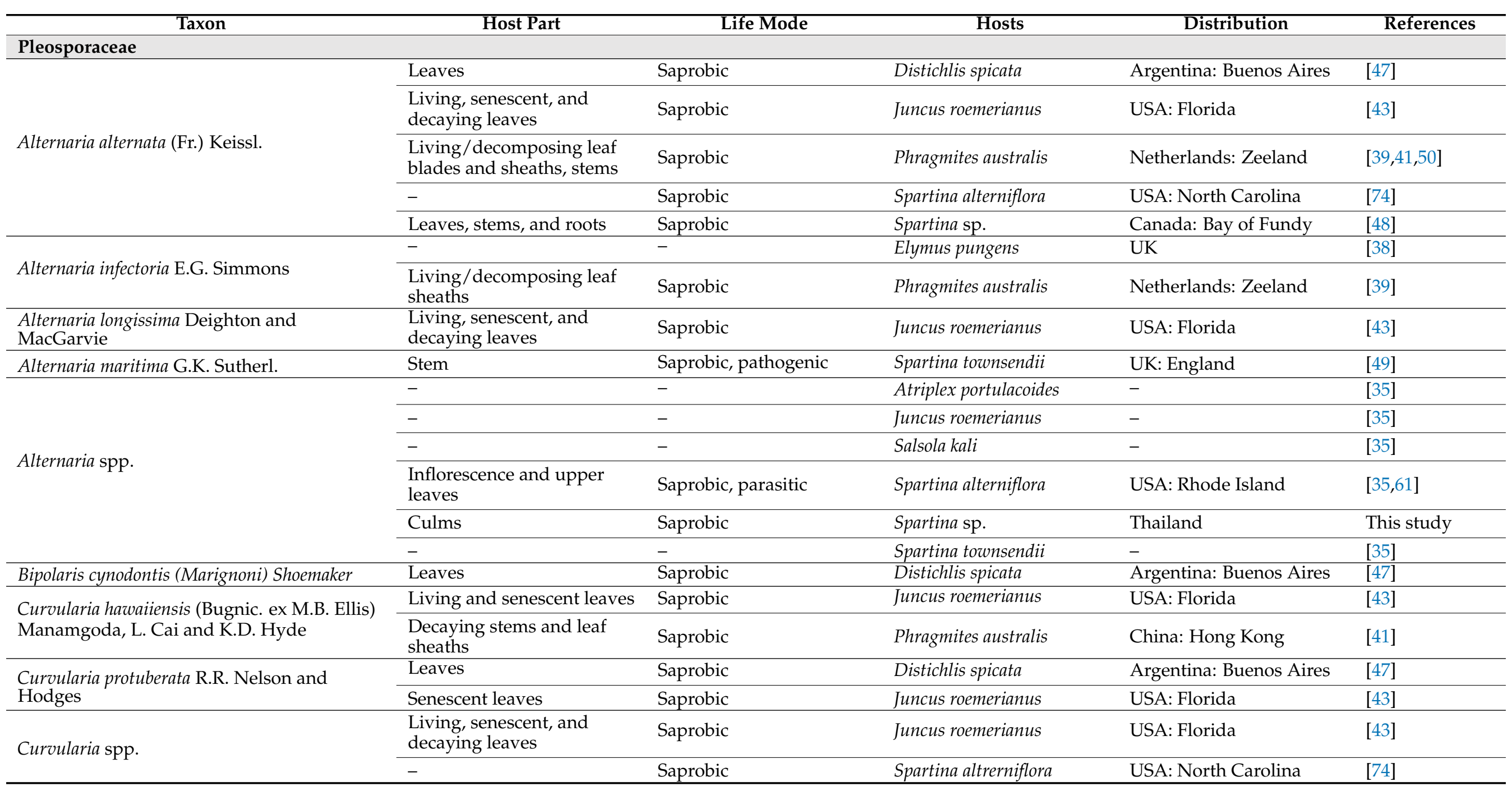


Table 1. Cont.

\begin{tabular}{|c|c|c|c|c|c|}
\hline Taxon & Host Part & Life Mode & Hosts & Distribution & References \\
\hline Curvularia tuberculata B.L. Jain & $\begin{array}{l}\text { Senescent and decaying } \\
\text { leaves }\end{array}$ & Saprobic & Juncus roemerianus & USA: Florida & [43] \\
\hline \multirow{8}{*}{$\begin{array}{l}\text { Decorospora gaudefroyi (Pat.) Inderb., Kohlm. } \\
\text { and Volkm.-Kohlm. }\end{array}$} & Stems & Saprobic & Atriplex sp. & UK: Portsmouth & [106] \\
\hline & - & - & Atriplex portulacoides & - & [35] \\
\hline & - & - & Sarcocornia perennis & - & [35] \\
\hline & - & - & Sarcoconia fructicosa & - & [35] \\
\hline & - & - & Salicornia europaea & - & [35] \\
\hline & - & - & Salicornia sp. & - & [35] \\
\hline & $\begin{array}{l}\text { Leaf sheaths and blades, } \\
\text { stem }\end{array}$ & Saprobic & Spartina maritima & Portugal: Algarve & [59] \\
\hline & - & - & Suaeda maritima & - & [35] \\
\hline Drechslera sp. & $\begin{array}{l}\text { Living, senescent, and } \\
\text { decaying leaves }\end{array}$ & Saprobic & Juncus roemerianus & USA: Florida & [43] \\
\hline \multirow{4}{*}{$\begin{array}{l}\text { Exserohilum rostratum (Drechsler) K.J. } \\
\text { Leonard and Suggs }\end{array}$} & - & - & Distichlis spicata & - & [35] \\
\hline & $\begin{array}{l}\text { Living, senescent, and } \\
\text { decaying leaves }\end{array}$ & Saprobic & Juncus roemerianus & USA: Florida & [43] \\
\hline & Senescent and dead leaves & Saprobic & Spartina alterniflora & $\begin{array}{l}\text { USA: Rhode Island, } \\
\text { North Carolina, Florida }\end{array}$ & {$[35,36,73]$} \\
\hline & - & - & Spartina spp. & - & [32] \\
\hline \multirow{2}{*}{$\begin{array}{l}\text { Paradendryphiella arenariae (Nicot) Woudenb. } \\
\text { and Crous }\end{array}$} & Decomposing culms & Saprobic & Spartina alterniflora & USA: Rhode Island & {$[35,61]$} \\
\hline & - & - & Spartina spp. & - & [32] \\
\hline \multirow{9}{*}{$\begin{array}{l}\text { Paradendryphiella salina (G.K. Sutherl.) } \\
\text { Woudenb. and Crous }\end{array}$} & - & - & Atriplex portulacoides & - & [35] \\
\hline & Decaying leaves & Saprobic & Juncus roemerianus & USA: Florida & [43] \\
\hline & - & - & Puccinellia maritima & - & [35] \\
\hline & - & - & Salicornia europaea & - & {$[35]$} \\
\hline & Decomposing culms & Saprobic & Spartina alterniflora & USA: Rhode Island & {$[35,61]$} \\
\hline & - & - & Spartina spp. & - & [32] \\
\hline & - & - & Spartina townsendii & - & [35] \\
\hline & Leaves and stems & Saprobic & Spartina sp. & Canada: Bay of Fundy & [48] \\
\hline & - & - & Suaeda maritima & - & [35] \\
\hline
\end{tabular}


Table 1. Cont.

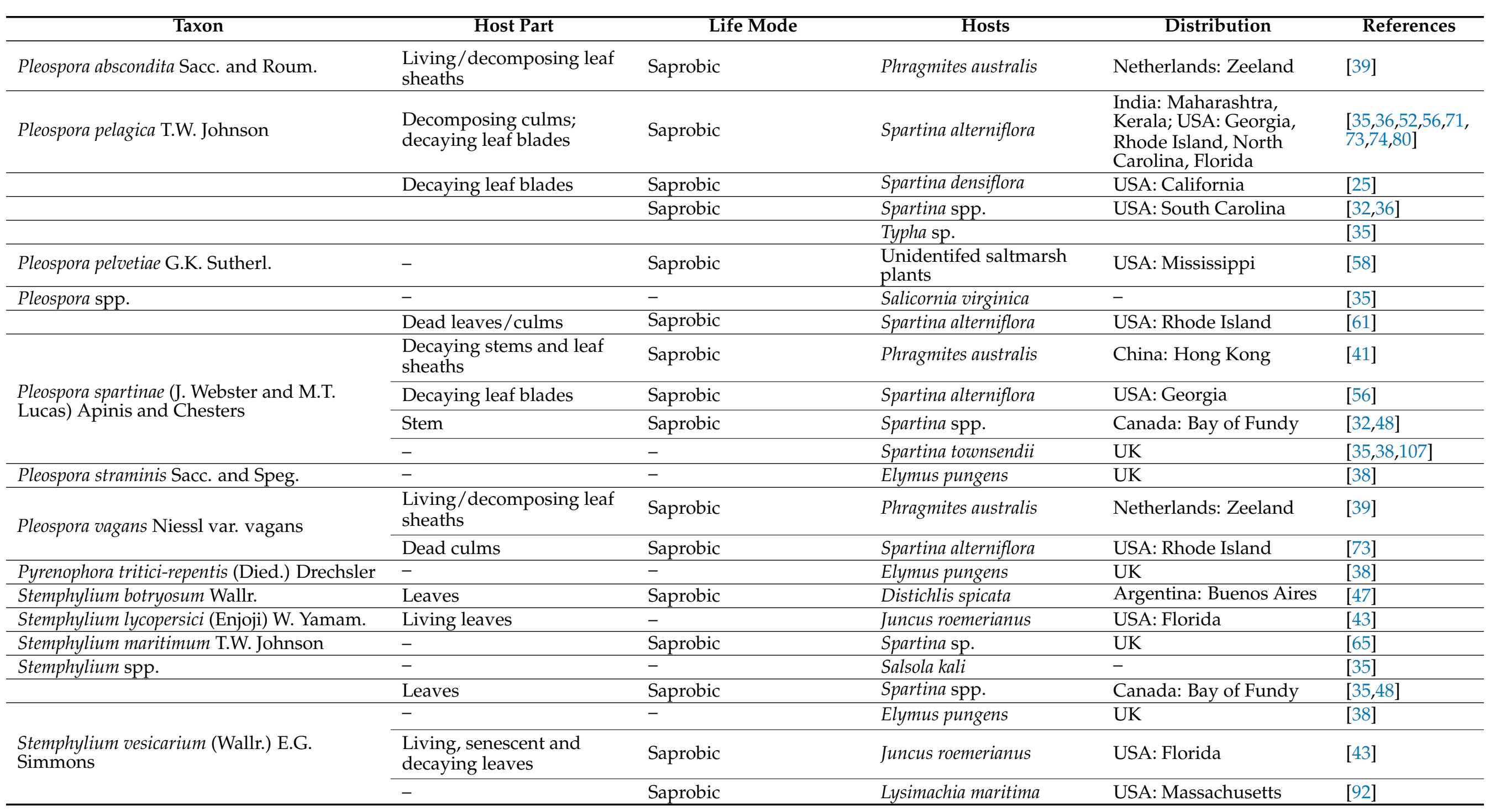


Table 1. Cont

\begin{tabular}{|c|c|c|c|c|c|}
\hline Taxon & Host Part & Life Mode & Hosts & Distribution & References \\
\hline & - & Saprobic & Spartina alterniflora & USA: Rhode Island & [61] \\
\hline & Glumes, rachis & - & Spartina townsendii & UK: England & {$[38,49]$} \\
\hline & & & Spartina sp. & UK & [65] \\
\hline \multirow[t]{2}{*}{$\begin{array}{l}\text { Stemphylium triglochinicola B. Sutton and } \\
\text { Piroz. }\end{array}$} & - & - & Triglochin maritima & Sweden: Västergötland & {$[35,87]$} \\
\hline & $\begin{array}{l}\text { Dead leaves and } \\
\text { inflorescences }\end{array}$ & Saprobic & Triglochin sp. & India: Kerala; UK & {$[52,108]$} \\
\hline \multirow{5}{*}{ Typhicola typharum (Desm.) Crous } & Senescent and dead leaves & Saprobic, pathogenic & Spartina alterniflora & $\begin{array}{l}\text { Canada; USA: Maine, } \\
\text { Rhode Island, } \\
\text { Connecticut, New Jersey, } \\
\text { Virginia, North Carolina, } \\
\text { Florida }\end{array}$ & {$[35,36,61,73,74]$} \\
\hline & - & Saprobic & Spartina patens & USA: Rhode Island & [36] \\
\hline & - & & Spartina townsendii & UK & [38] \\
\hline & - & Saprobic & Spartina spp. & $\begin{array}{l}\text { Argentina: Buenos Aires; } \\
\text { Canada; USA: Maine }\end{array}$ & {$[35,36]$} \\
\hline & Stems & Saprobic & Spartina townsendii & UK: England & {$[35,49,65]$} \\
\hline Phialophorophoma litoralis Linder & Stem and sheath & Saprobic & Spartina marítima & Portugal: Alentejo, Lisbon & {$[54,63]$} \\
\hline Phialophorophoma spp. & $\begin{array}{l}\text { Living/decomposing leaf } \\
\text { sheaths, stems }\end{array}$ & Saprobic & Phragmites australis & Netherlands: Zeeland & {$[39,40]$} \\
\hline Pyrenochaeta sp. & Living leaves & Saprobic & Juncus roemerianus & USA: Florida & {$[43]$} \\
\hline $\begin{array}{l}\text { Scolecobasidium humicola G.L. Barron and L.V. } \\
\text { Busch }\end{array}$ & $\begin{array}{l}\text { Living, senescent, and } \\
\text { decaying leaves }\end{array}$ & Saprobic & Juncus roemerianus & USA: Florida & {$[43]$} \\
\hline \multicolumn{6}{|l|}{ Roussoellaceae } \\
\hline Cytoplea sp. & $\begin{array}{l}\text { Decaying stems and leaf } \\
\text { sheaths }\end{array}$ & Saprobic & Phragmites australis & China: Hong Kong & {$[41]$} \\
\hline \multicolumn{6}{|l|}{ Sporormiaceae } \\
\hline Preussia funiculata (Preuss) Fuckel & - & - & Spartina townsendii & UK & {$[38]$} \\
\hline Preussia terricola Cain & - & - & Elymus pungens & UK & [38] \\
\hline Sporormia longipes Massee and E.S. Salmon & - & - & Elymus pungens & UK & [38] \\
\hline Sporormia sp. & $\begin{array}{l}\text { Senescent and } \\
\text { decaying leaves }\end{array}$ & Saprobic & Juncus roemerianus & USA: Florida & [43] \\
\hline
\end{tabular}


Table 1. Cont.

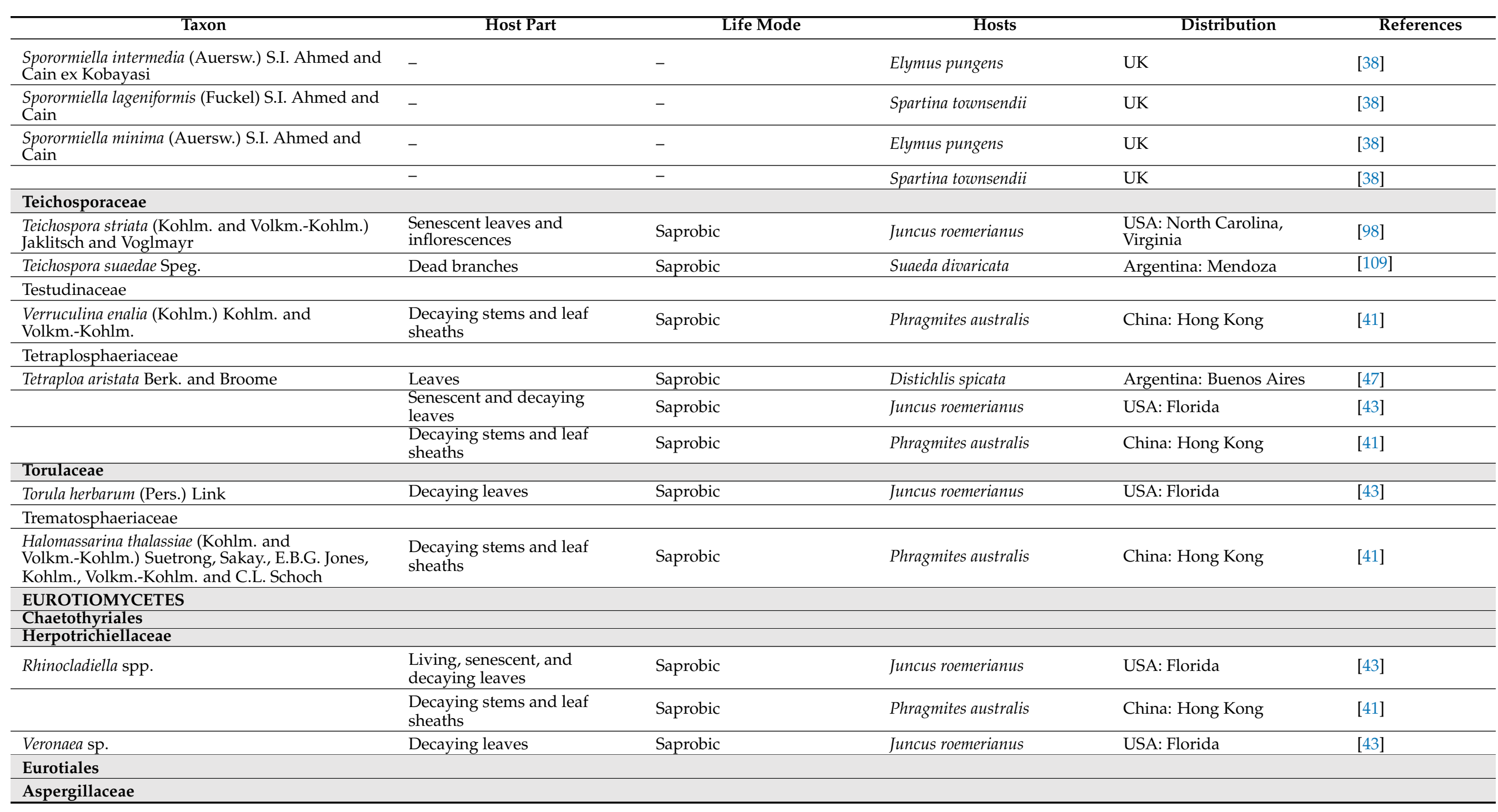


Table 1. Cont.

\begin{tabular}{|c|c|c|c|c|c|}
\hline Taxon & Host Part & Life Mode & Hosts & Distribution & References \\
\hline Aspergillus fumigatus Fresen. & - & - & Elymus pungens & UK & {$[38]$} \\
\hline \multirow{2}{*}{ Aspergillus nidulans (Eidam) G. Winter } & - & - & Elymus pungens & UK & {$[38]$} \\
\hline & - & - & Spartina townsendii & UK & {$[38]$} \\
\hline Aspergillus niger Tiegh. & $\begin{array}{l}\text { Living, senescent, and } \\
\text { decaying leaves }\end{array}$ & Saprobic & Juncus roemerianus & USA: Florida & {$[43]$} \\
\hline \multirow[t]{2}{*}{ Aspergillus spp. } & $\begin{array}{l}\text { Living, senescent, and } \\
\text { decaying leaves }\end{array}$ & Saprobic & Juncus roemerianus & USA: Florida & [43] \\
\hline & - & - & Spartina townsendii & UK: England & [49] \\
\hline Monascus purpureus Went & - & - & Elymus pungens & UK & {$[38]$} \\
\hline Penicillium aurantiogriseum Dierckx & Leaves & Saprobic & Spartina sp. & Canada: Bay of Fundy & [48] \\
\hline Penicillium brevicompactum Dierckx & Roots & Saprobic & Spartina sp. & Canada: Bay of Fundy & [48] \\
\hline Penicillium chrysogenum Thom & Roots & Saprobic & Spartina sp. & Canada: Bay of Fundy & {$[48]$} \\
\hline Penicillium lividum Westling & Leaves and stems & Saprobic & Spartina sp. & Canada: Bay of Fundy & {$[48]$} \\
\hline \multirow[b]{2}{*}{ Penicillium spp. } & $\begin{array}{l}\text { Living, senescent, and } \\
\text { decaying leaves }\end{array}$ & Saprobic & Juncus roemerianus & USA: Florida & {$[43]$} \\
\hline & $\begin{array}{l}\text { Decaying stems and leaf } \\
\text { sheaths }\end{array}$ & Saprobic & Phragmites australis & China: Hong Kong & [41] \\
\hline $\begin{array}{l}\text { Thermoascus crustaceus (Apinis and Chesters) } \\
\text { Stolk }\end{array}$ & - & - & Elymus pungens & UK & [38] \\
\hline \multirow[t]{3}{*}{ Paecilomyces spp. } & $\begin{array}{l}\text { Senescent and decaying } \\
\text { leaves }\end{array}$ & Saprobic & Juncus roemerianus & USA: Florida & {$[43]$} \\
\hline & $\begin{array}{l}\text { Decaying stems and leaf } \\
\text { sheaths }\end{array}$ & Saprobic & Phragmites australis & China: Hong Kong & {$[41]$} \\
\hline & - & Saprobic & Salt marsh plants & India: Goa & {$[52]$} \\
\hline \multicolumn{6}{|l|}{ Trichocomaceae } \\
\hline $\begin{array}{l}\text { Thermomyces dupontii (Griffon and Maubl.) } \\
\text { Houbraken and Samson }\end{array}$ & - & - & Elymus pungens & UK & {$[38]$} \\
\hline \multicolumn{6}{|l|}{ Onygenales } \\
\hline \multicolumn{6}{|l|}{ Onygenaceae } \\
\hline Amauroascus albicans (Apinis) Arx & - & - & Elymus pungens & UK & {$[38]$} \\
\hline Amauroascus albicans (Apinis) Arx & - & - & Spartina townsendii & UK & [38] \\
\hline
\end{tabular}


Table 1. Cont.

\begin{tabular}{|c|c|c|c|c|c|}
\hline Taxon & Host Part & Life Mode & Hosts & Distribution & References \\
\hline \multicolumn{6}{|l|}{ LECANOROMYCETES } \\
\hline \multicolumn{6}{|l|}{ Ostropales } \\
\hline \multirow{2}{*}{$\begin{array}{l}\text { Glomerobolus gelineus Kohlm. and Volkm.-Kohlm. } \\
\text { Stictis sp. }\end{array}$} & Senescent culms & Saprobic & Juncus roemerianus & USA: North Carolina & [110] \\
\hline & $\begin{array}{l}\text { Living/decomposing leaf } \\
\text { sheaths }\end{array}$ & Saprobic & Phragmites australis & Netherlands: Zeeland & [39] \\
\hline \multicolumn{6}{|l|}{ LEOTIOMYCETES } \\
\hline \multicolumn{6}{|l|}{ Helotiales } \\
\hline \multicolumn{6}{|l|}{ Amorphothecaceae } \\
\hline Amorphotheca resinae Parbery & Roots & Saprobic & Spartina sp. & Canada: Bay of Fundy & [48] \\
\hline \multicolumn{6}{|l|}{ Calloriaceae } \\
\hline Cistella fugiens (W. Phillips) Matheis & Living/decomposing stems & Saprobic & Phragmites australis & Netherlands: Zeeland & {$[40]$} \\
\hline \multicolumn{6}{|l|}{ Helotiaceae } \\
\hline Cyathicula culmicola (Desm.) De Not. & - & - & Elymus pungens & UK & {$[38]$} \\
\hline Helotium sp. & - & - & Elymus pungens & UK & [38] \\
\hline Brunnipila palearum (Desm.) Baral & - & - & Spartina townsendii & UK & [38] \\
\hline Lachnum controversum (Cooke) Rehm & - & - & Elymus pungens & UK & {$[38]$} \\
\hline \multirow{2}{*}{ Lachnum spartinae S.A. Cantrell } & Decaying leaf sheaths & Saprobic & Spartina alterniflora & USA: Georgia & {$[56,111]$} \\
\hline & - & - & Spartina spp. & - & [32] \\
\hline \multicolumn{6}{|l|}{ Mollisiaceae } \\
\hline Belonopsis atriella (Cooke) Lindau & - & - & Spartina cynosuroides & USA: Louisiana & {$[68,90,112]$} \\
\hline Mollisia hydrophila (P. Karst.) Sacc. & $\begin{array}{l}\text { Living/decomposing leaf } \\
\text { sheaths }\end{array}$ & Saprobic & Phragmites australis & Netherlands: Zeeland & {$[39]$} \\
\hline Mollisia palustris (P. Karst.) P. Karst. & $\begin{array}{l}\text { Living/decomposing leaf } \\
\text { sheaths }\end{array}$ & Saprobic & Phragmites australis & Netherlands: Zeeland & [39] \\
\hline Trichobelonium kneiffii (Wallr.) J. Schröt. & $\begin{array}{l}\text { Living/decomposing leaf } \\
\text { sheaths, stems }\end{array}$ & Saprobic & Phragmites australis & Netherlands: Zeeland & {$[39,40]$} \\
\hline \multicolumn{6}{|l|}{ Ploettnerulaceae } \\
\hline $\begin{array}{l}\text { Cadophora melinii Nannf. } \\
\text { Sclerotiniaceae }\end{array}$ & Leaves & Saprobic & Spartina sp. & Canada: Bay of Fundy & {$[48]$} \\
\hline
\end{tabular}


Table 1. Cont.

\begin{tabular}{|c|c|c|c|c|c|}
\hline Taxon & Host Part & Life Mode & Hosts & Distribution & References \\
\hline \multirow[t]{2}{*}{ Botrytis cinerea Pers. } & Stem & & Spartina townsendii & UK: England & [49] \\
\hline & Leaves & Saprobic & Spartina sp. & Canada: Bay of Fundy & [48] \\
\hline Monilia sp. & Decaying leaves & Saprobic & Juncus roemerianus & USA: Florida & [43] \\
\hline \multicolumn{6}{|l|}{ Solenopeziaceae } \\
\hline \multirow{3}{*}{ Halenospora varia (Anastasiou) E.B.G. Jones } & Senescent leaves & Saprobic & Juncus roemerianus & USA: Florida & [43] \\
\hline & Basal area of the sheath & Saprobic & Spartina densiflora & Argentina: Buenos Aires & [64] \\
\hline & - & - & Spartina spp. & - & [32] \\
\hline \multicolumn{6}{|l|}{ Helotiales genera incertae sedis } \\
\hline Cejpia hystrix (De Not.) Baral & - & - & Elymus pungens & UK & [38] \\
\hline Dactylaria sp. & $\begin{array}{l}\text { Decaying stems and leaf } \\
\text { sheaths }\end{array}$ & Saprobic & Phragmites australis & China: Hong Kong & [41] \\
\hline Crocicreas gramineum (Fr.) Fr. & - & - & Elymus pungens & UK & [38] \\
\hline \multicolumn{6}{|l|}{$\begin{array}{l}\text { Leotiales } \\
\text { Leotiales genera incertae sedis }\end{array}$} \\
\hline Flagellospora sp. & Living leaves & - & Juncus roemerianus & USA: Florida & [43] \\
\hline \multicolumn{6}{|l|}{$\begin{array}{l}\text { Flagellospora sp. } \\
\text { Rhytismatales }\end{array}$} \\
\hline Lophodermium arundinaceum (Schrad.) Chevall. & 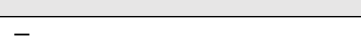 & - & Elymus pungens & UK & [38] \\
\hline \multirow{2}{*}{\multicolumn{6}{|c|}{$\begin{array}{l}\text { Thelebolales } \\
\text { Thelebolaceae }\end{array}$}} \\
\hline & & & & & \\
\hline \multirow{3}{*}{ Thelebolus crustaceus (Fuckel) Kimbr. } & - & - & Elymus pungens & UK & {$[38]$} \\
\hline & - & - & Puccinellia maritima & UK & [38] \\
\hline & - & - & Spartina townsendii & UK & {$[38]$} \\
\hline \multicolumn{6}{|l|}{ ORBILIOMYCETES } \\
\hline \multirow{2}{*}{\multicolumn{6}{|c|}{$\begin{array}{l}\text { Orbiliales } \\
\text { Orbiliaceae }\end{array}$}} \\
\hline & & & & & \\
\hline Arthrobotrys conoides Drechsler & $\begin{array}{l}\text { Decaying stems and leaf } \\
\text { sheaths }\end{array}$ & Saprobic & Phragmites australis & China: Hong Kong & [41] \\
\hline Arthrobotrys sp. & $\begin{array}{l}\text { Decaying stems and leaf } \\
\text { sheaths }\end{array}$ & Saprobic & Phragmites australis & China: Hong Kong & [41] \\
\hline Orbilia junci Kohlm., Baral and Volkm.-Kohlm. & Tips of senescent leaves & - & Juncus roemerianus & USA: North Carolina & [113] \\
\hline
\end{tabular}


Table 1. Cont.

Taxon

Host Part

Life Mode

Hosts

Distribution

References

\begin{tabular}{|c|c|c|c|c|c|}
\hline \multicolumn{6}{|l|}{ PEZIZOMYCETES } \\
\hline \multicolumn{6}{|l|}{ Pezizales } \\
\hline \multicolumn{6}{|l|}{ Pezizaceae } \\
\hline Belonium heteromorphum (Ellis and Everh.) Seaver & - & - & Spartina cynosuroides & USA: Louisiana & {$[68,114]$} \\
\hline \multicolumn{6}{|l|}{ SACCHAROMYCETES } \\
\hline \multicolumn{6}{|l|}{ Saccharomycetales } \\
\hline \multicolumn{6}{|l|}{ Debaryomycetaceae } \\
\hline $\begin{array}{l}\text { Debaryomyces hansenii (Zopf) Lodder and } \\
\text { Kreger-van Rij }\end{array}$ & Decaying leaf blades & Saprobic & Spartina alterniflora & USA: Louisiana & [56] \\
\hline $\begin{array}{l}\text { Scheffersomyces spartinae (Ahearn, Yarrow and } \\
\text { Meyers) Kurtzman and M. Suzuki }\end{array}$ & Decaying leaf blades & Saprobic & Spartina alterniflora & USA: Louisiana & [56] \\
\hline \multicolumn{6}{|l|}{ Saccharomycetaceae } \\
\hline Kluyveromyces lactis (Stell.-Dekk.) Van der Walt & Decaying leaf blades & Saprobic & Spartina alterniflora & USA: Louisiana & [56] \\
\hline \multicolumn{6}{|l|}{ SORDARIOMYCETES } \\
\hline \multicolumn{6}{|l|}{ Amphisphaeriales } \\
\hline \multicolumn{6}{|l|}{ Amphisphaeriaceae } \\
\hline Massariella sp. & - & - & Spartina townsendii & UK & [38] \\
\hline $\begin{array}{l}\text { Ommatomyces coronatus Kohlm., Volkm.-Kohlm. } \\
\text { and O.E. Erikss. }\end{array}$ & $\begin{array}{l}\text { Lower parts of senescent } \\
\text { culms }\end{array}$ & Saprobic & Juncus roemerianus & USA: North Carolina & [97] \\
\hline Pestalotia sp. & $\begin{array}{l}\text { Living, senescent and } \\
\text { decaying leaves }\end{array}$ & Saprobic & Juncus roemerianus & USA: Florida & [43] \\
\hline \multicolumn{6}{|l|}{ Apiosporaceae } \\
\hline \multirow{2}{*}{ Arthrinium arundinis (Corda) Dyko and B. Sutton } & $\begin{array}{l}\text { Living/decomposing leaf } \\
\text { sheaths }\end{array}$ & Saprobic & Phragmites australis & Netherlands: Zeeland & [39] \\
\hline & Dead culms & Saprobic & Phragmites sp. & South Australia & [62] \\
\hline \multirow{3}{*}{ Arthrinium phaeospermum (Corda) M.B. Ellis } & $\begin{array}{l}\text { Living/decomposing leaf } \\
\text { blades and sheaths, stems }\end{array}$ & Saprobic & Phragmites australis & Netherlands: Zeeland & {$[39,40,50]$} \\
\hline & - & Saprobic & Spartina patens & USA: Rhode Island & [61] \\
\hline & $\begin{array}{l}\text { Inflorescence and upper } \\
\text { leaves }\end{array}$ & Saprobic & Spartina alterniflora & USA: Rhode Island & [36] \\
\hline
\end{tabular}


Table 1. Cont.

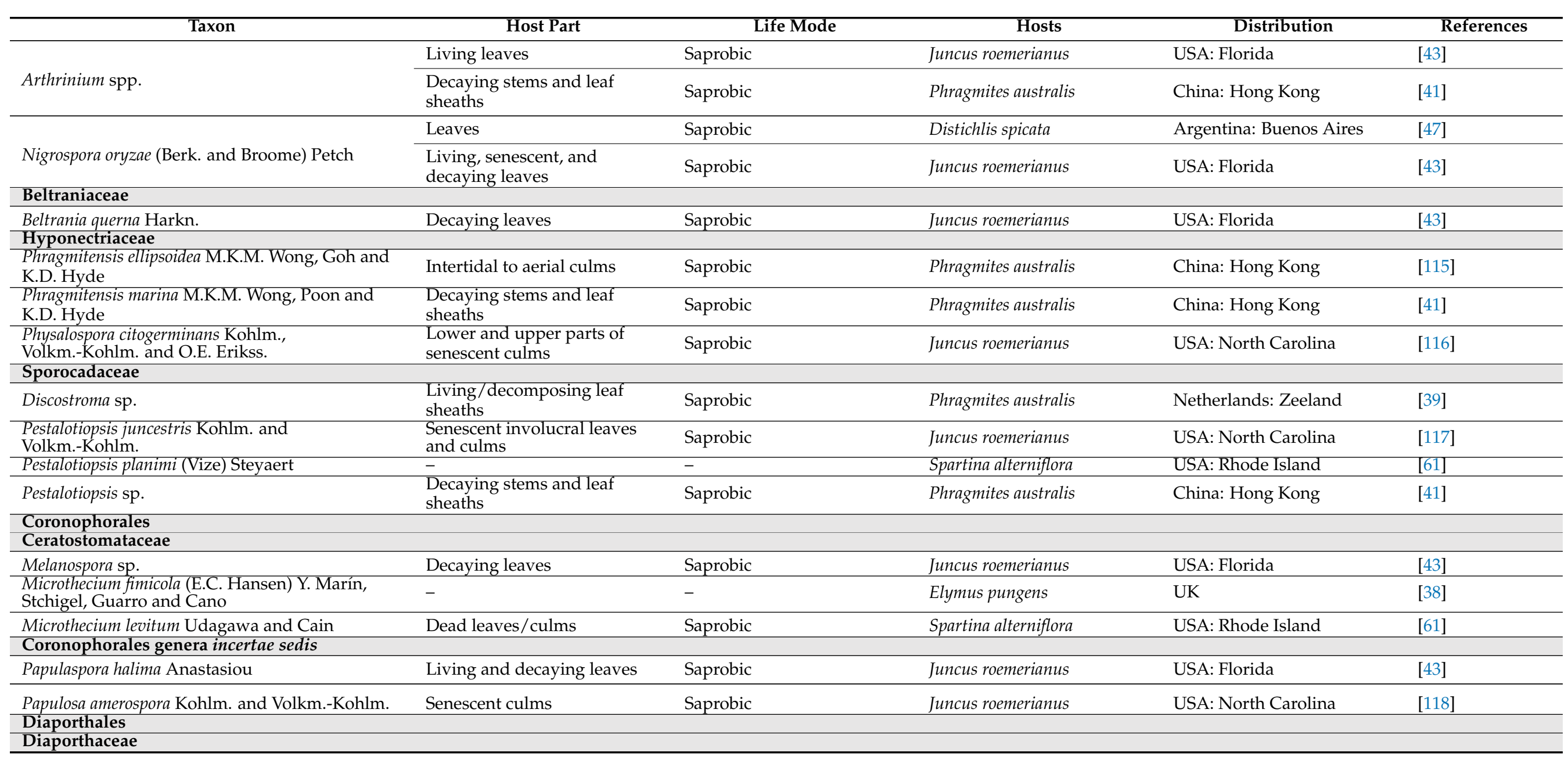


Table 1. Cont.

\begin{tabular}{|c|c|c|c|c|c|}
\hline Taxon & Host Part & Life Mode & Hosts & Distribution & References \\
\hline \multirow[t]{3}{*}{ Phomopsis spp. } & $\begin{array}{l}\text { Senescent and decaying } \\
\text { leaves }\end{array}$ & Saprobic & Juncus roemerianus & USA: Florida & [43] \\
\hline & $\begin{array}{l}\text { Decaying stems and leaf } \\
\text { sheaths }\end{array}$ & Saprobic & Phragmites australis & China: Hong Kong & [41] \\
\hline & - & - & Spartina sp. & - & [71] \\
\hline \multicolumn{6}{|l|}{ Gnomoniaceae } \\
\hline \multirow{3}{*}{$\begin{array}{l}\text { Gnomonia salina E.B.G. Jones (probably a nomen } \\
\text { dubiumand possibly a Halosarpheia species) }\end{array}$} & - & Saprobic & Spartina alterniflora & USA: Connecticut & [36] \\
\hline & & & Spartina spp. & & {$[32,35]$} \\
\hline & - & - & Spartina townsendii & UK & {$[35,65]$} \\
\hline \multicolumn{6}{|l|}{ Diaporthales incertae sedis } \\
\hline Botryodiplodia sp. & $\begin{array}{l}\text { Senescent and decaying } \\
\text { leaves }\end{array}$ & Saprobic & Juncus roemerianus & USA: Florida & [43] \\
\hline \multicolumn{6}{|l|}{$\begin{array}{l}\text { Glomerellales } \\
\text { Glomerellaceae }\end{array}$} \\
\hline Colletotrichum sp. & $\begin{array}{l}\text { Decaying stems and leaf } \\
\text { sheaths }\end{array}$ & Saprobic & Phragmites australis & China: Hong Kong & [41] \\
\hline \multicolumn{6}{|l|}{ Plectosphaerellaceae } \\
\hline Stachylidium bicolor Link & Senescent leaves & Saprobic & Juncus roemerianus & USA: Florida & [43] \\
\hline \multicolumn{6}{|l|}{$\begin{array}{l}\text { Hypocreales } \\
\text { Bionectriaceae }\end{array}$} \\
\hline \multirow[t]{3}{*}{ Acremonium spp. } & Leaves & Saprobic & Distichlis spicata & Argentina: Buenos Aires & [47] \\
\hline & $\begin{array}{l}\text { Living, senescent, and } \\
\text { decaying leaves }\end{array}$ & Saprobic & Juncus roemerianus & USA: Florida & [43] \\
\hline & $\begin{array}{l}\text { Decaying stems and leaf } \\
\text { sheaths }\end{array}$ & Saprobic & Phragmites australis & China: Hong Kong & [41] \\
\hline $\begin{array}{l}\text { Clonostachys rosea (Link) Schroers, Samuels, } \\
\text { Seifert and W. Gams }\end{array}$ & Leaves & Saprobic & Spartina sp. & Canada: Bay of Fundy & [48] \\
\hline Fusariella obstipa (Pollack) S. Hughes & Decaying leaves & Saprobic & Juncus roemerianus & USA: Florida & [43] \\
\hline Gliomastix spp. & $\begin{array}{l}\text { Senescent and decaying } \\
\text { leaves }\end{array}$ & Saprobic & Juncus roemerianus & USA: Florida & [43] \\
\hline & $\begin{array}{l}\text { Decaying stems and leaf } \\
\text { sheaths }\end{array}$ & Saprobic & Phragmites australis & China: Hong Kong & [41] \\
\hline $\begin{array}{l}\text { midrule Hydropisphaera arenula (Berk. and } \\
\text { Broome) Rossman and Samuels }\end{array}$ & $\begin{array}{l}\text { Living/decomposing leaf } \\
\text { sheaths }\end{array}$ & Saprobic & Phragmites australis & Netherlands: Zeeland & [39] \\
\hline \multirow{2}{*}{$\begin{array}{l}\text { Hydropisphaera erubescens (Roberge ex Desm.) } \\
\text { Rossman and Samuels }\end{array}$} & Decaying leaf blades & Saprobic & Spartina alterniflora & USA: Georgia & [56] \\
\hline & - & - & Spartina spp. & - & [32] \\
\hline
\end{tabular}


Table 1. Cont.

Taxon

Host Part

Life Mode

Hosts

Distribution

References

\begin{tabular}{|c|c|c|c|c|c|}
\hline Taxon & Host Part & Life Mode & Hosts & Distribution & References \\
\hline \multicolumn{6}{|l|}{ Clavicipitaceae } \\
\hline Atkinsonella hypoxylon (Peck) Diehl & - & - & Spartina cynosuroides & - & [68] \\
\hline \multirow{6}{*}{ Claviceps purpurea (Fr.) Tul. } & - & Saprobic & Phragmites australis & $\begin{array}{l}\text { UK: England (Southampton } \\
\text { Hampshire, Sussex, Oxon) }\end{array}$ & {$[119,120]$} \\
\hline & - & Pathogenic & Spartina anglica & UK & [123] \\
\hline & - & Saprobic, parasitic & Spartina cynosuroides & $\begin{array}{l}\text { USA: New York, Florida, } \\
\text { Mississippi }\end{array}$ & {$[44,68,121,124]$} \\
\hline & & & Spartina patens & USA: Maryland, Mississippi & {$[44,68,124,125]$} \\
\hline & - & - & Spartina townsendii & UK: England & {$[120,126]$} \\
\hline & - & - & Spartina sp. & Argentina & [122] \\
\hline Claviceps sp. & - & - & Spartina foliosa & USA: California & [127] \\
\hline Metarhizium anisopliae (Metschn.) Sorokīn & Leaves & Saprobic & Distichlis spicata & Argentina: Buenos Aires & [47] \\
\hline \multicolumn{6}{|l|}{ Hypocreaceae } \\
\hline Cladobotryum sp. & Decaying leaves & Saprobic & Juncus roemerianus & USA: Florida & [43] \\
\hline $\begin{array}{l}\text { Trichoderma citrinum (Pers.) Jaklitsch, W. Gams } \\
\text { and Voglmayr }\end{array}$ & Leaves & Saprobic & Spartina sp. & Canada: Bay of Fundy & [48] \\
\hline Trichoderma sp. & $\begin{array}{l}\text { Decaying stems and leaf } \\
\text { sheaths }\end{array}$ & Saprobic & Phragmites australis & China: Hong Kong & [41] \\
\hline Trichoderma viride Pers. & $\begin{array}{l}\text { Living, senescent, and } \\
\text { decaying leaves }\end{array}$ & Saprobic & Juncus roemerianus & USA: Florida & [43] \\
\hline \multicolumn{6}{|l|}{ Nectriaceae } \\
\hline Calonectria sp. & - & - & Elymus pungens & UK & [38] \\
\hline Fusarium fujikuroi Nirenberg & - & Saprobic & Suaeda australis & South Australia & [62] \\
\hline Fusarium graminearum Schwabe & $\begin{array}{l}\text { Living/decomposing leaf } \\
\text { sheaths, stems }\end{array}$ & Saprobic & Phragmites australis & Netherlands: Zeeland & {$[39,40]$} \\
\hline Fusarium heterosporum Nees and T. Nees & - & - & Spartina maritima & - & [128] \\
\hline
\end{tabular}


Table 1. Cont.

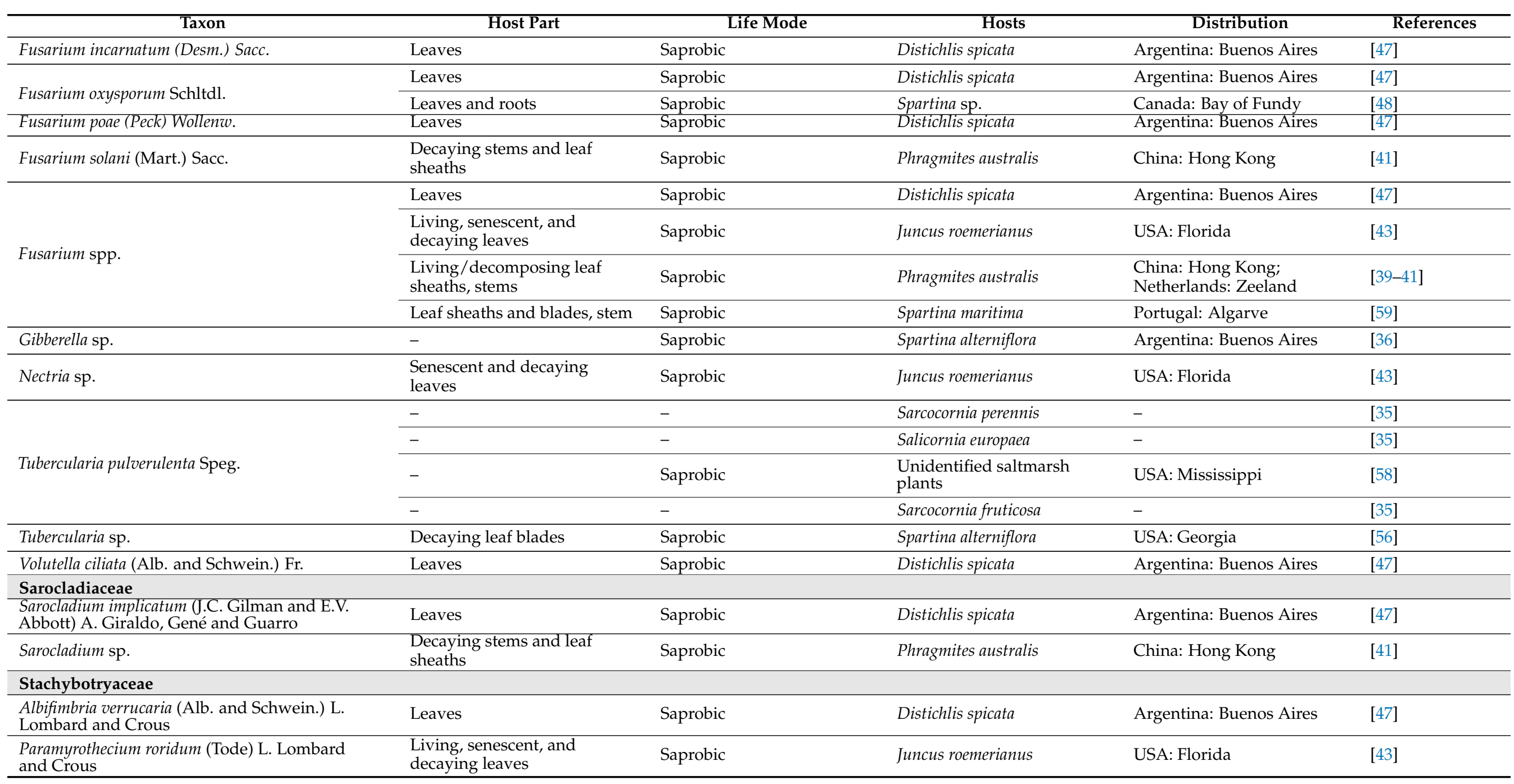


Table 1. Cont.

\section{Taxon}

Stachybotrys chartarum (Ehrenb.) S. Hughes Stachybotrys cylindrosporus C.N. Jensen

Stachybotrys echinatus (Rivolta) G. Sm.

Stachybotrys kampalensis Hansf.

Stachybotrys nephrosporus Hansf.

Stachybotrys spp

Striaticonidium cinctum (Corda) L. Lombard and

Crous

Xepicula jollymannii (N.C. Preston) L. Lombard

and Crous

Hypocreales genera incertae sedis

Cephalosporium spp.

Lulworthiales

\section{Lulworthiaceae}

Cumulospora marina I. Schmidt

Halazoon fuscus (I. Schmidt) Abdel-Wahab, K.L.

Pang, Nagah., Abdel-Aziz and E.B.G. Jones

Halazoon melhae Abdel-Aziz, Abdel-Wahab and Nagahama

Lulworthia floridana Meyers

Life Mode

Host Part

Senescent and decaying

Senescen
leaves

Hosts

Decaying leaves Saprobic

Senescent and decaying

leaves

Senescent leaves

Saprobic

Saprobic

leaves

Senescent and decaying

leaves

Decaying stems and leaf

sheaths

Decaying leaf blade

Saprobic

Living/decomposing leaf sheaths

Senescent and decaying

leaves

Saprobic

Dead leaves/culms

Saprobic

Saprobic

Saprobic

Saprobic

Juncus roemerianus

Juncus roemerianus

Juncus roemerianus

Juncus roemerianus

Juncus roemerianus

Phragmites australis

Spartina alterniflora

Phragmites australis

Juncus roemerianus

Saprobic

Spartina alterniflora

USA

mead culm

repher

\begin{tabular}{lllll} 
Dead culm & Saprobic & Phragmites australis & $\begin{array}{l}\text { Iraq, Egypt, Germany, } \\
\text { Thailand }\end{array}$ & [129] \\
\hline- & - & Spartina spp. & - & [32] \\
\hline Decaying rhizomes & Saprobic & Phragmites australis & France, Germany, Japan & [35,130] \\
\hline Rhizomes and culms & Saprobic & Phragmites sp. & Sweden & [87] \\
\hline Decaying stem & Saprobic & Phragmites australis & Egypt: Port Said & [130] \\
\hline- & Saprobic & Spartina alterniflora & $\begin{array}{l}\text { USA: North Carolina, } \\
\text { Rhode Island }\end{array}$ \\
\hline
\end{tabular}


Table 1. Cont.

\begin{tabular}{|c|c|c|c|c|c|}
\hline Taxon & Host Part & Life Mode & Hosts & Distribution & References \\
\hline \multirow{4}{*}{$\begin{array}{l}\text { Lulworthia medusa (Ellis and Everh.) Cribb and } \\
\text { J.W. Cribb }\end{array}$} & - & - & Elymus pungens & UK & {$[38]$} \\
\hline & - & Saprobic & Spartina cynosuroides & USA: New Jersey & {$[89,132]$} \\
\hline & - & & Spartina spp. & USA: New Jersey & {$[32,89]$} \\
\hline & Stems & Saprobic & Spartina townsendii & $\begin{array}{l}\text { UK: England (Wales); USA: } \\
\text { Virginia, North Carolina, } \\
\text { South Carolina, Florida, } \\
\text { Texas }\end{array}$ & $\begin{array}{l}{[38,49,71,72,89} \\
132-134]\end{array}$ \\
\hline \multirow{7}{*}{ Lulworthia spp. } & - & - & Elymus pungens & - & [35] \\
\hline & - & - & Juncus roemerianus & - & {$[35,36]$} \\
\hline & Dead culms & Saprobic & Spartina alterniflora & $\begin{array}{l}\text { Argentina: Buenos Aires; } \\
\text { USA: Rhode Island, North } \\
\text { Carolina }\end{array}$ & {$[35,36,61,73,74]$} \\
\hline & - & - & Spartina cynosuroides & - & [35] \\
\hline & - & Saprobic & Spartina sp. & $\begin{array}{l}\text { Argentina: Buenos Aires; } \\
\text { Canada; USA: Maine, North } \\
\text { Carolina }\end{array}$ & {$[36]$} \\
\hline & - & - & Spartina townsendii & - & [35] \\
\hline & Leaf sheaths and blades, stem & Saprobic & Spartina maritima & $\begin{array}{l}\text { Portugal: Alentejo, Lisbon, } \\
\text { Algarve, Centro }\end{array}$ & {$[31,54,59,63]$} \\
\hline $\begin{array}{l}\text { Moleospora maritima Abdel-Wahab, Abdel-Aziz } \\
\text { and Nagah. }\end{array}$ & Decayed stems & Saprobic & Phragmites australis & Egypt: Port Said & {$[130]$} \\
\hline \multicolumn{6}{|l|}{ Magnaporthales } \\
\hline \multicolumn{6}{|l|}{ Ceratosphaeriaceae } \\
\hline Ceratosphaeria sp. & Senescent leaves & Saprobic & Juncus roemerianus & USA: Florida & {$[43]$} \\
\hline \multicolumn{6}{|l|}{ Magnaporthaceae } \\
\hline Buergenerula spartinae Kohlm. and R.V. Gessner & $\begin{array}{l}\text { Lower stem and leaf sheath } \\
\text { during the growth phase of } \\
\text { the plant/living and dead; } \\
\text { decaying leaf blades }\end{array}$ & Saprobic, parasitic & Spartina alterniflora & $\begin{array}{l}\text { USA: Alabama, Rhode } \\
\text { Island, Maine, New } \\
\text { Hampshire, Connecticut, } \\
\text { Mississippi, New Jersey, } \\
\text { Virginia, North Carolina, } \\
\text { Florida, Georgia }\end{array}$ & $\begin{array}{l}{[20,35,36,55,56} \\
58,61,73,74,82,92]\end{array}$ \\
\hline
\end{tabular}


Table 1. Cont.

\begin{tabular}{|c|c|c|c|c|c|}
\hline Taxon & Host Part & Life Mode & Hosts & Distribution & References \\
\hline & Leaves & Saprobic & Spartina spp. & $\begin{array}{l}\text { Canada: Bay of Fundy; USA: } \\
\text { South Carolina; UK }\end{array}$ & $\begin{array}{l}{[32,35,36,48,65]} \\
\text { this study }\end{array}$ \\
\hline & Leaf sheaths and blades, stem & Saprobic & Spartina maritima & $\begin{array}{l}\text { Portugal: Alentejo, Lisbon, } \\
\text { Algarve, Centro }\end{array}$ & {$[31,54,59]$} \\
\hline Gaeumannomyces sp. & $\begin{array}{l}\text { Decaying stems and leaf } \\
\text { sheaths }\end{array}$ & Saprobic & Phragmites australis & China: Hong Kong & {$[41]$} \\
\hline $\begin{array}{l}\text { Kohlmeyeriopsis medullaris (Kohlm., } \\
\text { Volkm.-Kohlm. and O.E. Erikss.) Klaubauf, M.-H. } \\
\text { Lebrun and Crous }\end{array}$ & $\begin{array}{l}\text { Lower parts of senescent } \\
\text { culms }\end{array}$ & Saprobic & Juncus roemerianus & USA: North Carolina & {$[97,135]$} \\
\hline $\begin{array}{l}\text { Utrechtiana roumeguerei (Cavara) Videira and } \\
\text { Crous }\end{array}$ & $\begin{array}{l}\text { Living/decomposing leaf } \\
\text { blades and sheaths }\end{array}$ & Saprobic & Phragmites australis & Netherlands: Zeeland & {$[39,50]$} \\
\hline \multicolumn{6}{|l|}{ Pseudohalonectriaceae } \\
\hline Pseudohalonectria falcata Shearer & $\begin{array}{l}\text { Decaying stems and leaf } \\
\text { sheaths }\end{array}$ & Saprobic & Phragmites australis & China: Hong Kong & {$[41]$} \\
\hline $\begin{array}{l}\text { Pseudohalonectria halophila Kohlm. and } \\
\text { Volkm.-Kohlm. }\end{array}$ & $\begin{array}{l}\text { Fragments of leaves and } \\
\text { culms in the wrack }\end{array}$ & Saprobic & Juncus roemerianus & USA: North Carolina & {$[105]$} \\
\hline \multicolumn{6}{|l|}{ Meliolales } \\
\hline \multicolumn{6}{|l|}{ Meliolaceae } \\
\hline \multicolumn{6}{|l|}{ Microascales } \\
\hline \multicolumn{6}{|l|}{ Halosphaeriaceae } \\
\hline \multirow[t]{5}{*}{ Aniptodera chesapeakensis Shearer and M.A. Mill. } & Dead leaves & Saprobic & Juncus roemerianus & USA: North Carolina & [35] \\
\hline & $\begin{array}{l}\text { Decaying stems and leaf } \\
\text { sheaths }\end{array}$ & Saprobic & Phragmites australis & China: Hong Kong & {$[41]$} \\
\hline & - & - & Spartina alterniflora & - & [35] \\
\hline & - & - & Spartina spp. & - & [32] \\
\hline & Leaf sheaths and blades, stem & Saprobic & Spartina maritima & $\begin{array}{l}\text { Portugal: Alentejo, Algarve, } \\
\text { Centro }\end{array}$ & {$[59,63]$} \\
\hline Aniptodera juncicola Volkm.-Kohlm. and Kohlm. & Dead standing culms of & Saprobic & Juncus roemerianus & $\begin{array}{l}\text { India: Kerala, West Bengal, } \\
\text { Tamil Nadu; USA: North } \\
\text { Carolina }\end{array}$ & {$[52,136]$} \\
\hline Aniptodera phragmiticola O. K. Poon et K. D. Hyde & $\begin{array}{l}\text { Decaying stems and leaf } \\
\text { sheaths }\end{array}$ & Saprobic & Phragmites australis & China: Hong Kong & [41] \\
\hline
\end{tabular}


Table 1. Cont.

\begin{tabular}{|c|c|c|c|c|c|}
\hline Taxon & Host Part & Life Mode & Hosts & Distribution & References \\
\hline \multirow[t]{5}{*}{ Ceriosporopsis halima Linde } & - & - & Arundo donax & - & {$[35]$} \\
\hline & Submerged seeds & Saprobic & Spartina alterniflora & USA & [137] \\
\hline & - & - & Spartina spp. & - & [32] \\
\hline & - & - & Spartina townsendii & UK & {$[35,38]$} \\
\hline & Stem & Saprobic & Spartina maritima & Portugal: Alentejo & {$[63]$} \\
\hline \multirow{4}{*}{$\begin{array}{l}\text { Cirrenalia macrocephala (Kohlm.) Meyers and } \\
\text { R.T. Moore }\end{array}$} & - & - & Ammophila arenaria & - & {$[35]$} \\
\hline & Decaying culms & Saprobic & Juncus roemerianus & USA: Florida & [43] \\
\hline & - & - & Spartina spp. & - & [32] \\
\hline & Stem & Saprobic & Spartina maritima & Portugal: Alentejo & [63] \\
\hline Cirrenalia pseudomacrocephala Kohlm. & Senescent leaves & Saprobic & Juncus roemerianus & USA: Florida & [43] \\
\hline \multirow{3}{*}{ Corollospora maritima Werderm. } & $\begin{array}{l}\text { Submerged seeds, } \\
\text { decomposing culms }\end{array}$ & Saprobic & Spartina alterniflora & USA: Rhode Island & {$[20,35,61,137]$} \\
\hline & - & - & Spartina spp. & - & [32] \\
\hline & Stem & Saprobic & Spartina maritima & Portugal: Alentejo & [63] \\
\hline \multirow{2}{*}{$\begin{array}{l}\text { Corollospora ramulosa (Meyers and Kohlm.) E.B.G. } \\
\text { Jones and Abdel-Wahab }\end{array}$} & - & Saprobic & $\begin{array}{l}\text { Unidentified saltmarsh } \\
\text { plants }\end{array}$ & USA: Mississippi & {$[58]$} \\
\hline & - & Saprobic & Zostera marina & USA: North Carolina & [74] \\
\hline \multirow{3}{*}{ Haligena elaterophora Kohlm. } & - & - & Spartina alterniflora & - & {$[35]$} \\
\hline & - & - & Spartina tonwsendii & UK & {$[38]$} \\
\hline & - & - & Spartina spp. & - & [32] \\
\hline $\begin{array}{l}\text { Halosarpheia culmiperda Kohlm., Volkm.-Kohlm. } \\
\text { and O.E. Erikss. }\end{array}$ & $\begin{array}{l}\text { Lower parts of senescent } \\
\text { culms }\end{array}$ & Saprobic & Juncus roemerianus & USA: North Carolina & [97] \\
\hline Halosarpheia sp. & Stem & Saprobic & Spartina maritima & Portugal: Alentejo & [63] \\
\hline \multirow{2}{*}{$\begin{array}{l}\text { Halosarpheia viscosa I. Schmidt ex Shearer and } \\
\text { J.L. Crane }\end{array}$} & Decaying leaf blades & Saprobic & Spartina alterniflora & USA: Georgia & {$[56]$} \\
\hline & - & Saprobic & Spartina maritima & Portugal: Lisbon & [54] \\
\hline Halosphaeria appendiculata Linder & - & - & Arundo donax & - & [35] \\
\hline Halosphaeria sp. & Submerged seeds & Saprobic & Spartina alterniflora & USA & [137] \\
\hline $\begin{array}{l}\text { Lautisporopsis circumvestita (Kohlm.) E.B.G. Jones, } \\
\text { Yusoff and S.T. Moss }\end{array}$ & - & - & Arundo donax & - & [35] \\
\hline
\end{tabular}


Table 1. Cont.

\begin{tabular}{|c|c|c|c|c|c|}
\hline Taxon & Host Part & Life Mode & Hosts & Distribution & References \\
\hline \multirow{4}{*}{ Lignincola laevis Höhnk } & - & - & Elymus pungens & - & [35] \\
\hline & - & Saprobic & Spartina spp. & USA: North Carolina & {$[32,138]$} \\
\hline & - & - & Spartina townsendii & - & [35] \\
\hline & Stem & Saprobic & Spartina maritima & Portugal: Alentejo & [63] \\
\hline \multirow{7}{*}{$\begin{array}{l}\text { Magnisphaera spartinae (E.B.G. Jones) J. Campb., } \\
\text { J.L. Anderson and Shearer }\end{array}$} & - & - & Elymus farctus & - & {$[35]$} \\
\hline & - & - & Elymus pungens & - & [35] \\
\hline & Living/decomposing stems & Saprobic & Phragmites australis & Netherlands: Zeeland & [40] \\
\hline & - & & Spartina spp. & & [32] \\
\hline & - & Saprobic & Spartina patens & USA: Rhode Island & [36] \\
\hline & Stem & Saprobic & Spartina townsendii & UK: Wales & {$[35,139]$} \\
\hline & - & & Typha sp. & & [35] \\
\hline \multirow[t]{2}{*}{ Nais inornata Kohlm. } & Decomposing culms & Saprobic & Spartina alterniflora & USA: Rhode Island & {$[20,35,61]$} \\
\hline & & & Spartina spp. & & [32] \\
\hline $\begin{array}{l}\text { Natantispora unipolaris K.L. Pang, S.Y. Guo and } \\
\text { E.B.G. Jones }\end{array}$ & Dead stem & Saprobic & Phragmites australis & Taiwan: Nankunshen & [140] \\
\hline \multirow{2}{*}{$\begin{array}{l}\text { Oceanitis unicaudata (E.B.G. Jones and } \\
\text { Camp.-Als.) J. Dupont and E.B.G. Jones }\end{array}$} & $\begin{array}{l}\text { Decaying stems and leaf } \\
\text { sheaths }\end{array}$ & Saprobic & Phragmites australis & China: Hong Kong & [41] \\
\hline & Stem & Saprobic & Spartina maritima & Portugal: Alentejo & [63] \\
\hline $\begin{array}{l}\text { Panorbis viscosus (I. Schmidt) J. Campb., J.L. } \\
\text { Anderson and Shearer }\end{array}$ & Leaf sheaths and blades, stem & Saprobic & Spartina maritima & Portugal: Alentejo, Algarve & {$[59,63]$} \\
\hline \multirow{3}{*}{ Remispora hamata (Höhnk) Kohlm. } & - & - & Elymus pungens & UK & {$[35,38]$} \\
\hline & $\begin{array}{l}\text { Senescent and decaying } \\
\text { leaves }\end{array}$ & Saprobic & Juncus roemerianus & USA: Florida & [43] \\
\hline & $\begin{array}{l}\text { Living/decomposing leaf } \\
\text { blades and sheaths, stems }\end{array}$ & Saprobic & Phragmites australis & Netherlands: Zeeland & {$[39,40,50]$} \\
\hline
\end{tabular}


Table 1. Cont.

\begin{tabular}{|c|c|c|c|c|c|}
\hline Taxon & Host Part & Life Mode & Hosts & Distribution & References \\
\hline & - & Saprobic & Phragmites sp. & Sweden & [87] \\
\hline & Dead leaves & Saprobic & Spartina alterniflora & $\begin{array}{l}\text { USA: Rhode Island, Maine, } \\
\text { Florida }\end{array}$ & {$[20,35,36,61,73]$} \\
\hline & - & Saprobic & Spartina sp. & $\begin{array}{l}\text { USA: North Carolina; } \\
\text { Argentina: Buenos Aires }\end{array}$ & {$[36,138]$} \\
\hline & - & - & Spartina townsendii & - & [35] \\
\hline & - & - & Typha sp. & - & [35] \\
\hline Remispora trullifera Kohlm. & Leaf sheaths and blades, stem & Saprobic & Spartina maritima & Portugal: Centro & [59] \\
\hline Tirispora unicaudata E.B.G. Jones and Vrijmoed & Stem & Saprobic & Spartina maritima & Portugal: Alentejo & [63] \\
\hline \multicolumn{6}{|l|}{ Microascaceae } \\
\hline Scopulariopsis spp. & $\begin{array}{l}\text { Living, senescent, and } \\
\text { decaying leaves }\end{array}$ & Saprobic & Juncus roemerianus & USA: Florida & {$[43]$} \\
\hline \multicolumn{6}{|l|}{ Myrmecridiales } \\
\hline \multicolumn{6}{|l|}{ Myrmecridiaceae } \\
\hline $\begin{array}{l}\text { Myrmecridium schulzeri (Sacc.) Arzanlou, W. } \\
\text { Gams and Crous }\end{array}$ & Leaves & Saprobic & Distichlis spicata & Argentina: Buenos Aires & {$[47]$} \\
\hline \multicolumn{6}{|l|}{ Ophiostomataceae } \\
\hline Sporothrix sp. & Senescent leaves & Saprobic & Juncus roemerianus & USA: Florida & [43] \\
\hline \multicolumn{6}{|l|}{ Phomatosporales } \\
\hline \multicolumn{6}{|l|}{ Phomatosporaceae } \\
\hline $\begin{array}{l}\text { Phomatospora bellaminuta Kohlm., Volkm.-Kohlm. } \\
\text { and O.E. Erikss. }\end{array}$ & $\begin{array}{l}\text { Lower parts of senescent } \\
\text { culms }\end{array}$ & Saprobic & Juncus roemerianus & USA: North Carolina & [116] \\
\hline Phomatospora berkeleyi Sacc. & $\begin{array}{l}\text { Living/decomposing leaf } \\
\text { blades and sheaths, stems }\end{array}$ & Saprobic & Phragmites australis & Netherlands: Zeeland & {$[39,40,50]$} \\
\hline \multirow{3}{*}{ Phomatospora dinemasporium J. Webster } & $\begin{array}{l}\text { Decaying stems and leaf } \\
\text { sheaths, stems }\end{array}$ & Saprobic & Phragmites australis & $\begin{array}{l}\text { China: Hong Kong; } \\
\text { Netherlands: Zeeland }\end{array}$ & {$[40,41]$} \\
\hline & Dead leaves & Saprobic & Phragmites sp. & South Australia & [62] \\
\hline & - & - & Spartina townsendii & UK & [38] \\
\hline Phomatospora phragmiticola Poon and K.D. Hyde & $\begin{array}{l}\text { Decaying stems and leaf } \\
\text { sheaths }\end{array}$ & Saprobic & Phragmites australis & China: Hong Kong & {$[41]$} \\
\hline
\end{tabular}


Table 1. Cont.

\begin{tabular}{|c|c|c|c|c|c|}
\hline Taxon & Host Part & Life Mode & Hosts & Distribution & References \\
\hline \multirow[t]{2}{*}{ Phomatospora spp. } & $\begin{array}{l}\text { Senescent and decaying } \\
\text { leaves }\end{array}$ & Saprobic & Juncus roemerianus & USA: Florida & [43] \\
\hline & $\begin{array}{l}\text { Living/decomposing leaf } \\
\text { sheaths, stems }\end{array}$ & Saprobic & Phragmites australis & Netherlands: Zeeland & {$[39,40]$} \\
\hline \multicolumn{6}{|l|}{ Phyllachorales } \\
\hline \multicolumn{6}{|l|}{ Phyllachoraceae } \\
\hline \multirow{3}{*}{ Phyllachora graminis (Pers.) Fuckel } & - & - & Elymus pungens & UK & {$[38]$} \\
\hline & - & Saprobic, pathogenic & Spartina alterniflora & USA: Massachusetts & {$[44]$} \\
\hline & - & - & Spartina cynosuroides & - & [68] \\
\hline \multirow{2}{*}{ Phyllachora cynodontis Niessl. } & - & Saprobic, pathogenic & Spartina alterniflora & USA & {$[68]$} \\
\hline & - & Saprobic, pathogenic & Spartina foliosa & USA: California & {$[44,112,141]$} \\
\hline Phyllachora paludicola Kohlm. and Volkm.-Kohlm. & $\begin{array}{l}\text { Dead leaves (lower half of } \\
\text { standing culms) }\end{array}$ & Saprobic & Spartina alterniflora & $\begin{array}{l}\text { USA: Florida, Georgia, } \\
\text { North Carolina, Maryland, } \\
\text { Delaware }\end{array}$ & [142] \\
\hline Phyllachora sylvatica Sacc. and Speg. & - & Saprobic & Spartina patens & USA: South Carolina & [141] \\
\hline \multicolumn{6}{|l|}{ Savoryellales } \\
\hline \multicolumn{6}{|l|}{ Savoryellaceae } \\
\hline \multirow{4}{*}{$\begin{array}{l}\text { Savoryella paucispora (Cribb and J.W. Cribb) J. } \\
\text { Koch }\end{array}$} & - & - & Juncus roemerianus & - & [35] \\
\hline & - & - & Spartina alterniflora & - & [35] \\
\hline & - & - & Spartina sp. & - & [35] \\
\hline & - & - & Spartina townsendii & - & [35] \\
\hline \multicolumn{6}{|l|}{ Sordariales } \\
\hline \multicolumn{6}{|l|}{ Chaetomiaceae } \\
\hline Achaetomium sp. & Decaying leaves & Saprobic & Juncus roemerianus & USA: Florida & [43] \\
\hline \multirow{4}{*}{ Chaetomium elatum Kunze } & - & - & Elymus pungens & UK & [38] \\
\hline & - & - & Puccinellia maritima & UK & {$[38]$} \\
\hline & - & - & Spartina townsendii & UK & {$[38]$} \\
\hline & - & - & & & \\
\hline \multirow[b]{2}{*}{ Chaetomium globosum Kunze } & - & - & Elymus pungens & UK & {$[38]$} \\
\hline & $\begin{array}{l}\text { Decaying stems and leaf } \\
\text { sheaths }\end{array}$ & Saprobic & Phragmites australis & China: Hong Kong & {$[41]$} \\
\hline
\end{tabular}




\begin{tabular}{|c|c|c|c|c|c|}
\hline Taxon & Host Part & Life Mode & Hosts & Distribution & References \\
\hline & - & - & Puccinellia maritima & UK & [38] \\
\hline & - & - & Spartina townsendii & UK & [38] \\
\hline Chaetomium spirale Zopf & - & - & Elymus pungens & UK & {$[38]$} \\
\hline \multirow{3}{*}{ Chaetomium thermophilum La Touche } & - & - & Elymus pungens & UK & {$[38]$} \\
\hline & - & - & Puccinellia maritima & UK & [38] \\
\hline & - & - & Spartina townsendii & UK & [38] \\
\hline Chaetomium sp. & Stem & Saprobic & Typha sp. & UK & This study \\
\hline \multirow[t]{2}{*}{ Corynascus sepedonium (C.W. Emmons) Arx } & - & - & Puccinellia maritima & UK & {$[38]$} \\
\hline & - & - & Spartina townsendii & UK & [38] \\
\hline \multirow{3}{*}{$\begin{array}{l}\text { Dichotomopilus funicola (Cooke) X.Wei Wang } \\
\text { and Samson }\end{array}$} & - & - & Elymus pungens & UK & {$[38]$} \\
\hline & - & - & Spartina alterniflora & USA: Rhode Island & [61] \\
\hline & - & - & Spartina townsendii & UK & [38] \\
\hline $\begin{array}{l}\text { Dichotomopilus indicus (Corda) X.Wei Wang } \\
\text { and Samson }\end{array}$ & - & - & Elymus pungens & UK & [38] \\
\hline \multirow{2}{*}{ Humicola sp. } & $\begin{array}{l}\text { Living, senescent, and } \\
\text { decaying leaves }\end{array}$ & Saprobic & Juncus roemerianus & USA: Florida & [43] \\
\hline & $\begin{array}{l}\text { Decaying stems and leaf } \\
\text { sheaths }\end{array}$ & Saprobic & Phragmites australis & China: Hong Kong & {$[41]$} \\
\hline \multirow{2}{*}{$\begin{array}{l}\text { Thermothielavioides terrestris (Apinis) X. Wei Wang } \\
\text { and Houbraken }\end{array}$} & - & - & Elymus pungens & UK & {$[38]$} \\
\hline & - & - & Puccinellia maritima & UK & [38] \\
\hline Trichocladium constrictum I. Schmidt & Stem & Saprobic & Spartina maritima & Portugal: Alentejo & [63] \\
\hline \multirow{2}{*}{$\begin{array}{l}\text { Trichocladium crispatum (Fuckel) X. Wei Wang and } \\
\text { Houbraken }\end{array}$} & - & - & Elymus pungens & UK & {$[38]$} \\
\hline & - & - & Spartina townsendii & UK & {$[38]$} \\
\hline Lasiosphaeriaceae & - & - & & & \\
\hline Schizothecium hispidulum (Speg.) N. Lundq. & $\begin{array}{l}\text { Living/decomposing leaf } \\
\text { sheaths }\end{array}$ & Saprobic & Phragmites australis & Netherlands: Zeeland & {$[39]$} \\
\hline Zopfiella latipes (N. Lundq.) Malloch and Cain & $\begin{array}{l}\text { Decaying stems and leaf } \\
\text { sheaths }\end{array}$ & Saprobic & Phragmites australis & China: Hong Kong & [41] \\
\hline \multicolumn{6}{|l|}{ Sordariaceae } \\
\hline $\begin{array}{l}\text { Neurospora calospora (Mouton) Dania García, } \\
\text { Stchigel and Guarro }\end{array}$ & - & - & Elymus pungens & UK & {$[38]$} \\
\hline
\end{tabular}


Table 1. Cont.

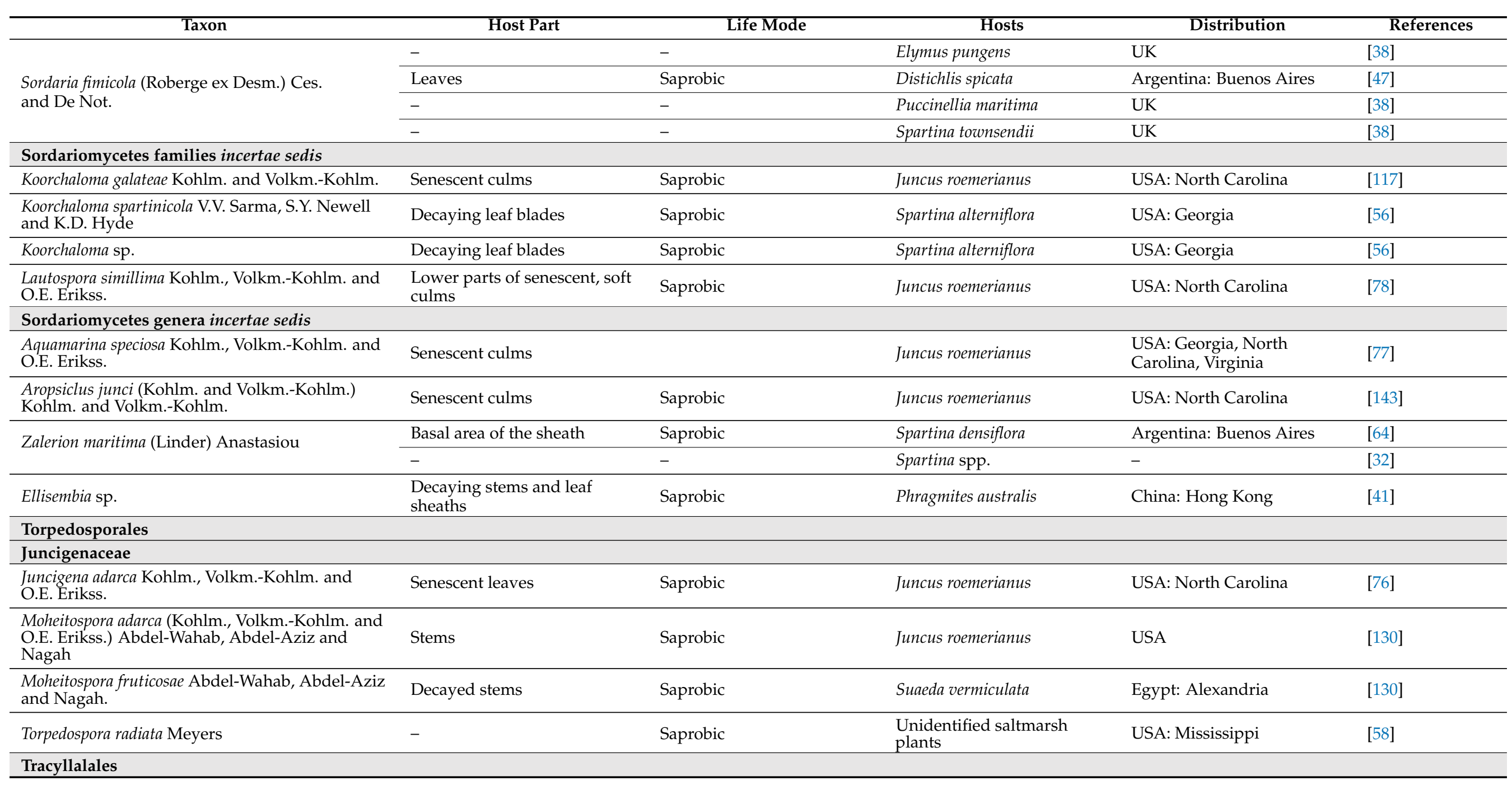


Table 1. Cont.

\begin{tabular}{|c|c|c|c|c|c|}
\hline Taxon & Host Part & Life Mode & Hosts & Distribution & References \\
\hline \multicolumn{6}{|l|}{ Tracyllaceae } \\
\hline Tracylla spartinae (Peck) Tassi & - & Saprobic, pathogenic & Spartina patens & USA: Mississippi & {$[44,68]$} \\
\hline \multicolumn{6}{|l|}{ Xylariales } \\
\hline \multicolumn{6}{|l|}{ Diatrypaceae } \\
\hline Cryptovalsa suaedicola Spooner & Dead twigs & Saprobic & Suaeda vermiculata & UK: Great Britain & [144] \\
\hline Halocryptovalsa salicorniae Dayar. and K.D. Hyde & Dead stem & Saprobic & Salicornia sp. & $\begin{array}{l}\text { Thailand: Prachuap Khiri } \\
\text { Khan }\end{array}$ & [145] \\
\hline \multicolumn{6}{|l|}{ Xylariaceae } \\
\hline $\begin{array}{l}\text { Anthostomella atroalba Kohlm., Volkm.-Kohlm. } \\
\text { and O.E. Erikss. }\end{array}$ & Senescent leaves & Saprobic & Juncus roemerianus & USA: North Carolina & {$[60]$} \\
\hline Anthostomella lugubris (Roberge ex Desm.) Sacc. & - & - & Elymus pungens & UK & [38] \\
\hline Anthostomella phaeosticta (Berk.) Sacc. & - & - & Elymus pungens & UK & [38] \\
\hline $\begin{array}{l}\text { Anthostomella poecila Kohlm., Volkm.-Kohlm. and } \\
\text { O.E. Erikss. }\end{array}$ & $\begin{array}{l}\text { Lower and upper parts of } \\
\text { senescent culms, decaying } \\
\text { leaves }\end{array}$ & Saprobic & Juncus roemerianus & $\begin{array}{l}\text { USA: Alabama, Mississippi, } \\
\text { North Carolina }\end{array}$ & {$[55,58,116]$} \\
\hline $\begin{array}{l}\text { Anthostomella punctulata (Roberge ex Desm.) } \\
\text { Sacc. }\end{array}$ & $\begin{array}{l}\text { Living/decomposing leaf } \\
\text { blades and sheaths }\end{array}$ & Saprobic & Phragmites australis & Netherlands: Zeeland & {$[39,50]$} \\
\hline $\begin{array}{l}\text { Anthostomella semitecta Kohlm., Volkm.-Kohlm. } \\
\text { and O.E. Erikss. }\end{array}$ & Senescent culms & - & Juncus roemerianus & USA: North Carolina & [116] \\
\hline \multirow{3}{*}{$\begin{array}{l}\text { Anthostomella spissitecta Kohlm. and } \\
\text { Volkm.-Kohlm. }\end{array}$} & $\begin{array}{l}\text { Leaf sheaths of senescent } \\
\text { culms }\end{array}$ & Saprobic & $\begin{array}{l}\text { Spartina alterniflora, } S \text {. } \\
\text { densiflora. }\end{array}$ & $\begin{array}{l}\text { USA: Connecticut, Florida, } \\
\text { North Carolina, Rhode } \\
\text { Island; Argentina: Buenos } \\
\text { Aires }\end{array}$ & [32] \\
\hline & - & - & Spartina sp. & - & [32] \\
\hline & Leaf sheaths and blades, stem & Saprobic & Spartina maritima & Portugal: Algarve & [59] \\
\hline \multirow{3}{*}{ Anthostomella spp. } & - & - & Elymus pungens & UK & [38] \\
\hline & - & Saprobic & Spartina alterniflora & $\begin{array}{l}\text { USA: Connecticut, Florida, } \\
\text { North Carolina, Rhode } \\
\text { Island; Argentina }\end{array}$ & {$[36,61]$} \\
\hline & - & - & Spartina townsendii & UK & [38] \\
\hline Anthostomella torosa Kohlm. and Volkm.-Kohlm. & $\begin{array}{l}\text { Senescent culms (restricted to } \\
\text { short culms) }\end{array}$ & Saprobic & Juncus roemerianus & USA: North Carolina & [32] \\
\hline Geniculosporium sp. & $\begin{array}{l}\text { Living, senescent, and } \\
\text { decaying leaves }\end{array}$ & Saprobic & Juncus roemerianus & USA: Florida & [43] \\
\hline
\end{tabular}


Table 1. Cont.

\begin{tabular}{|c|c|c|c|c|c|}
\hline Taxon & Host Part & Life Mode & Hosts & Distribution & References \\
\hline Rosellinia sp. & Dead leaves/culms & Saprobic & Spartina alterniflora & USA: Rhode Island & [61] \\
\hline Virgaria nigra (Link) Nees & Senescent leaves & Saprobic & Juncus roemerianus & USA: Florida & [43] \\
\hline \multicolumn{6}{|l|}{ Zygosporiaceae } \\
\hline Zygosporium masonii S. Hughes & Decaying leaves & Saprobic & Juncus roemerianus & USA: Florida & [43] \\
\hline Zygosporium sp. & Decaying leaves & Saprobic & Juncus roemerianus & USA: Florida & [43] \\
\hline \multicolumn{6}{|l|}{ Xylariales genera incertae sedis } \\
\hline Circinotrichum maculiforme Nees & Decaying leaves & Saprobic & Juncus roemerianus & USA: Florida & [43] \\
\hline \multicolumn{6}{|l|}{ Xylariomycetidae family incertae sedis } \\
\hline \multicolumn{6}{|l|}{ Cainiaceae } \\
\hline \multirow{2}{*}{ Atrotorquata lineata Kohlm. and Volkm.-Kohlm. } & Senescent culms & Saprobic & Juncus roemerianus & USA: North Carolina & [104] \\
\hline & & Saprobic & Unidentified saltmarsh plant & USA: Mississippi & [58] \\
\hline \multicolumn{6}{|l|}{ Ascomycota genera incertae sedis } \\
\hline \multirow{3}{*}{$\begin{array}{l}\text { Asteromyces cruciatus C. Moreau and Moreau ex } \\
\text { Hennebert }\end{array}$} & - & - & Agropyron sp. & - & [35] \\
\hline & - & - & Ammophila arenaria & - & [35] \\
\hline & - & Saprobic & Zostera sp. & USA: California & [74] \\
\hline $\begin{array}{l}\text { Cremasteria cymatilis Meyers and R.T. Moore } \\
\text { Nomen dubium }\end{array}$ & Senescent leaves & Saprobic & Juncus roemerianus & USA: Florida & [43] \\
\hline $\begin{array}{l}\text { Cytoplacosphaeria phragmiticola Poon and K.D. } \\
\text { Hyde }\end{array}$ & $\begin{array}{l}\text { Decaying stems and leaf } \\
\text { sheaths }\end{array}$ & Saprobic & Phragmites australis & China: Hong Kong & [41] \\
\hline Cytoplacosphaeria rimosa (Oudem.) Petr. & $\begin{array}{l}\text { Living/decomposing leaf } \\
\text { sheaths, stems }\end{array}$ & Saprobic & Phragmites australis & Netherlands: Zeeland & {$[39,40]$} \\
\hline Cytosporina sp. & $\begin{array}{l}\text { Living, senescent, and } \\
\text { decaying leaves }\end{array}$ & Saprobic & Juncus roemerianus & USA: Florida & [43] \\
\hline $\begin{array}{l}\text { Didymosamarospora euryhalina T.W. Johnson and } \\
\text { H.S. Gold }\end{array}$ & Culms & Saprobic & Juncus roemerianus & USA: North Carolina & [146] \\
\hline $\begin{array}{l}\text { Haplobasidion lelebae } \\
\text { Sawada ex M.B. Ellis }\end{array}$ & $\begin{array}{l}\text { Living, senescent, and } \\
\text { decaying leaves }\end{array}$ & Saprobic & Juncus roemerianus & USA: Florida & [43] \\
\hline $\begin{array}{l}\text { Hymenopsis chlorothrix Kohlm. and } \\
\text { Volkm.-Kohlm. }\end{array}$ & Senescent culms & Saprobic & Juncus roemerianus & USA: North Carolina & [147] \\
\hline $\begin{array}{l}\text { Hyphopolynema juncatile Kohlm. and } \\
\text { Volkm.-Kohlm. }\end{array}$ & Senescent leaves & Saprobic & Juncus roemerianus & USA: North Carolina & [148] \\
\hline
\end{tabular}


Table 1. Cont.

\begin{tabular}{|c|c|c|c|c|c|}
\hline Taxon & Host Part & Life Mode & Hosts & Distribution & References \\
\hline Kolletes undulatus Kohlm. and Volkm.-Kohlm. & Senescent leaves and culms & Saprobic & Juncus roemerianus & USA: North Carolina & [105] \\
\hline $\begin{array}{l}\text { Minimidochium parvum Cabello, } \\
\text { Aramb. and Cazau }\end{array}$ & Leaves & Saprobic & Distichlis spicata & Argentina: Buenos Aires & [47] \\
\hline \multirow[t]{2}{*}{ Monodictys pelagica (T. Johnson) E.B.G. Jones } & Decomposing culms & Saprobic & Spartina alterniflora & USA: Rhode Island & {$[20,35,61,73]$} \\
\hline & - & - & Spartina spp. & - & [32] \\
\hline Neottiospora sp. & $\begin{array}{l}\text { Living, senescent, and } \\
\text { decaying leaves }\end{array}$ & Saprobic & Juncus roemerianus & USA: Florida & {$[43]$} \\
\hline $\begin{array}{l}\text { Octopodotus stupendus Kohlm. and } \\
\text { Volkm.-Kohlm. }\end{array}$ & $\begin{array}{l}\text { Dead leaves (lower half of } \\
\text { standing culms) }\end{array}$ & Saprobic & Spartina alterniflora & USA: North Carolina & [142] \\
\hline Pycnodallia dupla Kohlm. and Volkm.-Kohlm. & $\begin{array}{l}\text { Senescent inflorescences } \\
\text { (involucral leaves and } \\
\text { branchlets) }\end{array}$ & Saprobic & Juncus roemerianus & USA: North Carolina & [147] \\
\hline Sphaeronaema sp. & Senescent leaves & Saprobic & Juncus roemerianus & USA: Florida & {$[43]$} \\
\hline Stauronema sp. & $\begin{array}{l}\text { Decaying stems and leaf } \\
\text { sheaths }\end{array}$ & Saprobic & Phragmites australis & China: Hong Kong & {$[41]$} \\
\hline Tetranacriella papillata Kohlm. and Volkm.-Kohlm. & Senescent leaves & Saprobic & Juncus roemerianus & USA: North Carolina & [117] \\
\hline Zythia spp. & $\begin{array}{l}\text { Living, senescent, and } \\
\text { decaying leaves }\end{array}$ & Saprobic & Juncus roemerianus & USA: Florida & {$[43]$} \\
\hline Psammina sp. & Senescent leaves & Saprobic & Juncus roemerianus & USA: Florida & [43] \\
\hline \multicolumn{6}{|l|}{ BASIDIOMYCOTA } \\
\hline \multicolumn{6}{|l|}{ AGARICOMYCETES } \\
\hline \multicolumn{6}{|l|}{ Agaricales } \\
\hline \multicolumn{6}{|l|}{ Niaceae } \\
\hline Merismodes bresadolae (Grelet) Singer & Living/decomposing stems & Saprobic & Phragmites australis & Netherlands: Zeeland & {$[40]$} \\
\hline Nia globispora Barata and Basilio & Stem & Saprobic & Spartina maritima & Portugal: Alentejo & [63] \\
\hline \multirow{3}{*}{ Nia vibrissa R.T. Moore and Meyers } & Old stem & Saprobic & Spartina alterniflora & USA: North Carolina & {$[35,149]$} \\
\hline & - & Saprobic & Spartina spp. & USA: North Carolina & {$[32,150]$} \\
\hline & Stem & Saprobic & Spartina maritima & Portugal: Alentejo & {$[63]$} \\
\hline
\end{tabular}


Table 1. Cont.

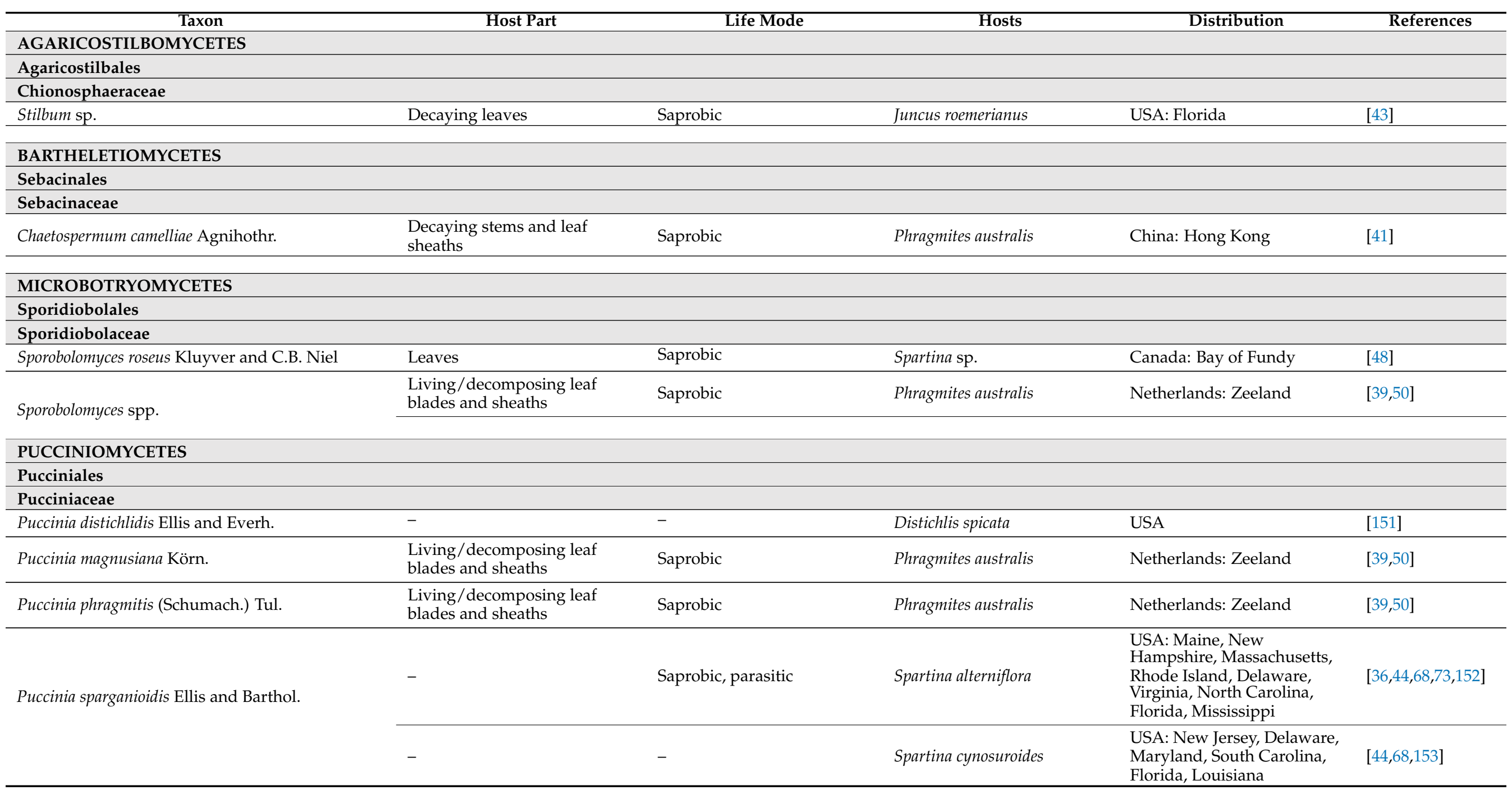


Table 1. Cont.

Taxon

Life Mode

Hosts

Distribution

References

Host Part

Saprobic, pathogenic

Spartina patens

USA: Connecticut,

Maryland, New Jersey, New $\quad[44,68,153]$
Yondicut, York

USA: Maine, New

Hampshire, Massachusetts,

Connecticut, New York,

New Jersey, Delaware,
Maryland, Florida

Uromyces acuminatus Arthur

Saprobic, pathogenic

Spartina alterniflora

USA: Florida

$[44,68,152]$

\begin{tabular}{ll}
\hline- & Saprobic, pathogenic \\
\hline
\end{tabular}

Spartina cynosuroides

USA: Connecticut, Delaware,

Florida, Maine, Maryland,

- Saprobic

Spartina patens

Massachusetts, New

Hampshire, New Jersey,

\begin{tabular}{|c|c|c|c|c|c|}
\hline Uromyces argutus F. Kern & - & Saprobic, pathogenic & Spartina alterniflora & France; USA: Florida & {$[44,68,152]$} \\
\hline Uromyces salicorniae (DC.) de Bary & - & - & Salicornia sp. & South Australia & [95] \\
\hline \multicolumn{6}{|l|}{ Pucciniales genera incertae sedis } \\
\hline Aecidium suaedae Thüm. & Leaves & - & Suaeda verae & Egypt & [154] \\
\hline
\end{tabular}

Aecidium suaedae Thüm

Leaves

Egypt

Phragmites australis

Netherlands: Zeeland

$[39,40]$

TREMELLOMYCETES

\section{Tremellales}

Tremellaceae

Tremella spicifera Van Ryck., Van de Put and

Living/decomposing leaf

Saprobic

ing: Zeeland

\section{USTILAGINOMYCETES}

Ustilaginales

Ustilaginaceae

\begin{tabular}{l}
\hline Tranzscheliella distichlidis (McAlpine) Vánky \\
\hline Ustilaginales genera incertae sedis
\end{tabular}

Parvulago marina (Durieu) R. Bauer, M. Lutz, $-$

Pathogenic

Distichlis spicata

Australia: Victoria

[155]

$-$

- Eleocharis parvula

Finland, France, Germany,

Urocystidales

Urocystidaceae 
Table 1. Cont.

\begin{tabular}{|c|c|c|c|c|c|}
\hline Taxon & Host Part & Life Mode & Hosts & Distribution & References \\
\hline $\begin{array}{l}\text { Flamingomyces ruppiae (Feldmann) R. Bauer, M. } \\
\text { Lutz, Piątek, Vánky and Oberw. }\end{array}$ & - & Parasitic & Ruppia maritima & France & [156] \\
\hline \multicolumn{6}{|l|}{ MUCOROMYCOTA } \\
\hline \multicolumn{6}{|l|}{ MUCOROMYCETES } \\
\hline \multicolumn{6}{|l|}{ Mucorales } \\
\hline \multicolumn{6}{|l|}{ Choanephoraceae } \\
\hline Blakeslea trispora Thaxt. & Senescent and decaying leaves & Saprobic & Juncus roemerianus & USA: Florida & [43] \\
\hline \multicolumn{6}{|l|}{ Mucoraceae } \\
\hline \multirow[t]{2}{*}{ Mucor sp. } & Senescent leaves & Saprobic & Juncus roemerianus & USA: Florida & [43] \\
\hline & Roots & Saprobic & Spartina sp. & Canada: Bay of Fundy & [48] \\
\hline \multicolumn{6}{|l|}{ Rhizopodaceae } \\
\hline Rhizopus stolonifer (Ehrenb.) Vuill. & Stems & Saprobic & Spartina townsendii & UK: England & [49] \\
\hline \multicolumn{6}{|l|}{ Syncephalastraceae } \\
\hline Syncephalastrum racemosum Cohn ex J. Schröt. & Living and senescent leaves & Saprobic & Juncus roemerianus & USA: Florida & [43] \\
\hline
\end{tabular}




\section{Taxonomic Classification of Salt Marsh Fungi}

\subsection{Phyla}

Calado and Barata [34] documented 332 taxa associated with Juncus roemerianus, Phragmites australis, and Spartina spp. In this review, we list 486 taxa that belong to three phyla (Ascomycota, Basidiomycota, Mucoromoycota) (Table 1, Figure 3) and selected species are illustrated in Figure 4. Ascomycota dominates the taxa from salt marsh ecosystems, accounting for $95.27 \%$ (463 taxa). Nineteen species in twelve genera (Aecidium, Chaetospermum, Falmingomyces, Merismodes, Nia, Parvulago, Puccinia, Sporobolomyces, Stilbum, Tranzscheliella, Tremella, Uromyces) belong to Basidiomycota (3.91\%), while Mucoromycota account for $0.82 \%$ (four species) of the salt marsh fungi.

\subsection{Class}

Salt marsh fungi are distributed into 17 classes (Table 1, Figure 5). Dothideomycetes has the highest number of taxa, which comprises $47.12 \%$ (229 taxa), followed by Sordariomycetes with 167 taxa (34.36\%). Twenty-one species (in 20 genera) can be referred to as Ascomycota genera incertae sedis. The Ascomycetes with the least number of species include Leotiomycetes (21 species, $4.32 \%$ ), Eurotiomycetes (16 species, $3.29 \%$ ), Orbiliomycetes ( 3 species, $0.62 \%$ ), Saccharomycetes ( 3 species, $0.62 \%$ ), Lecanoromycetes ( 2 species, $0.41 \%$ ), and Pezizomycetes (1 species, $0.21 \%$ ).

Seven classes represent the Basidiomycota (Figure 5). Puccinomycetes has the highest number of taxa documented (eight species, three genera) followed by Agaricomycetes (three species, two genera), Ustilaginomycetes (three species, three genera), and Microbotryomycetes (two taxa, one genus). Agaricostilbomycetes, Bartheletiomycetes, and Tremellomycetes have one representative taxon each.

The Mucoromoycota account for the taxa Blakeslea trispora, Mucor sp., Rhizopus stolonifera, and Syncephalastrum racemosum $[43,48,49]$.

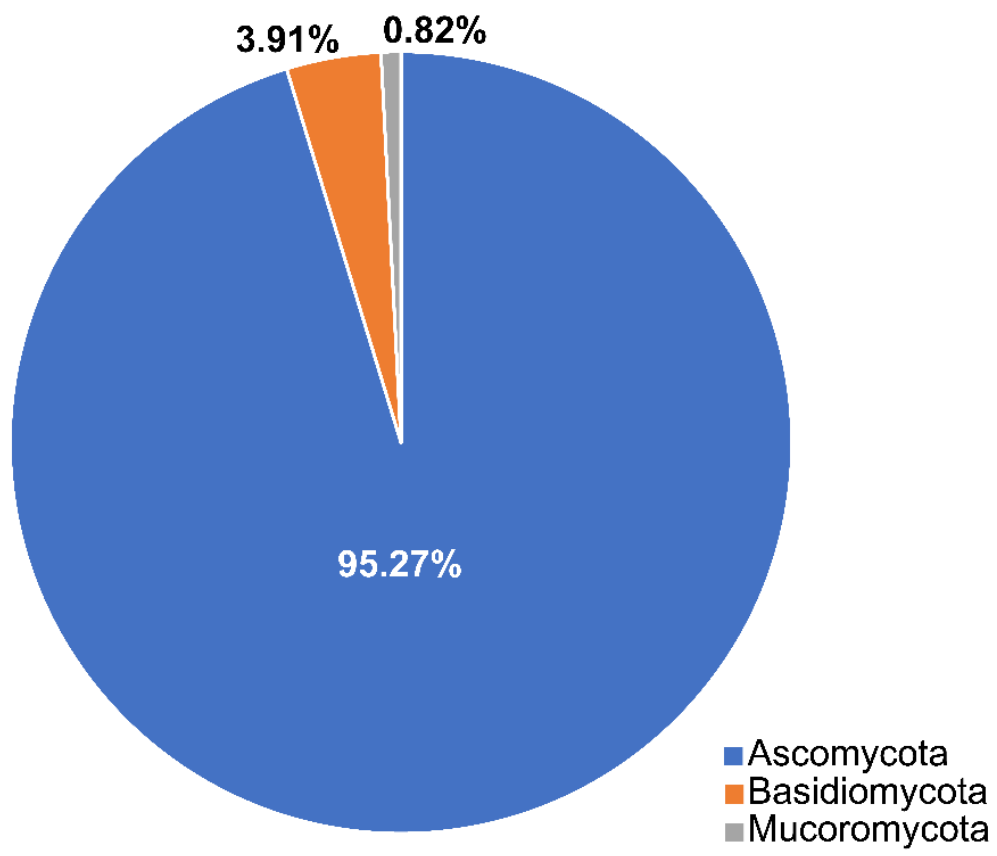

Figure 3. The distribution of salt marsh fungi among three fungal phyla. 


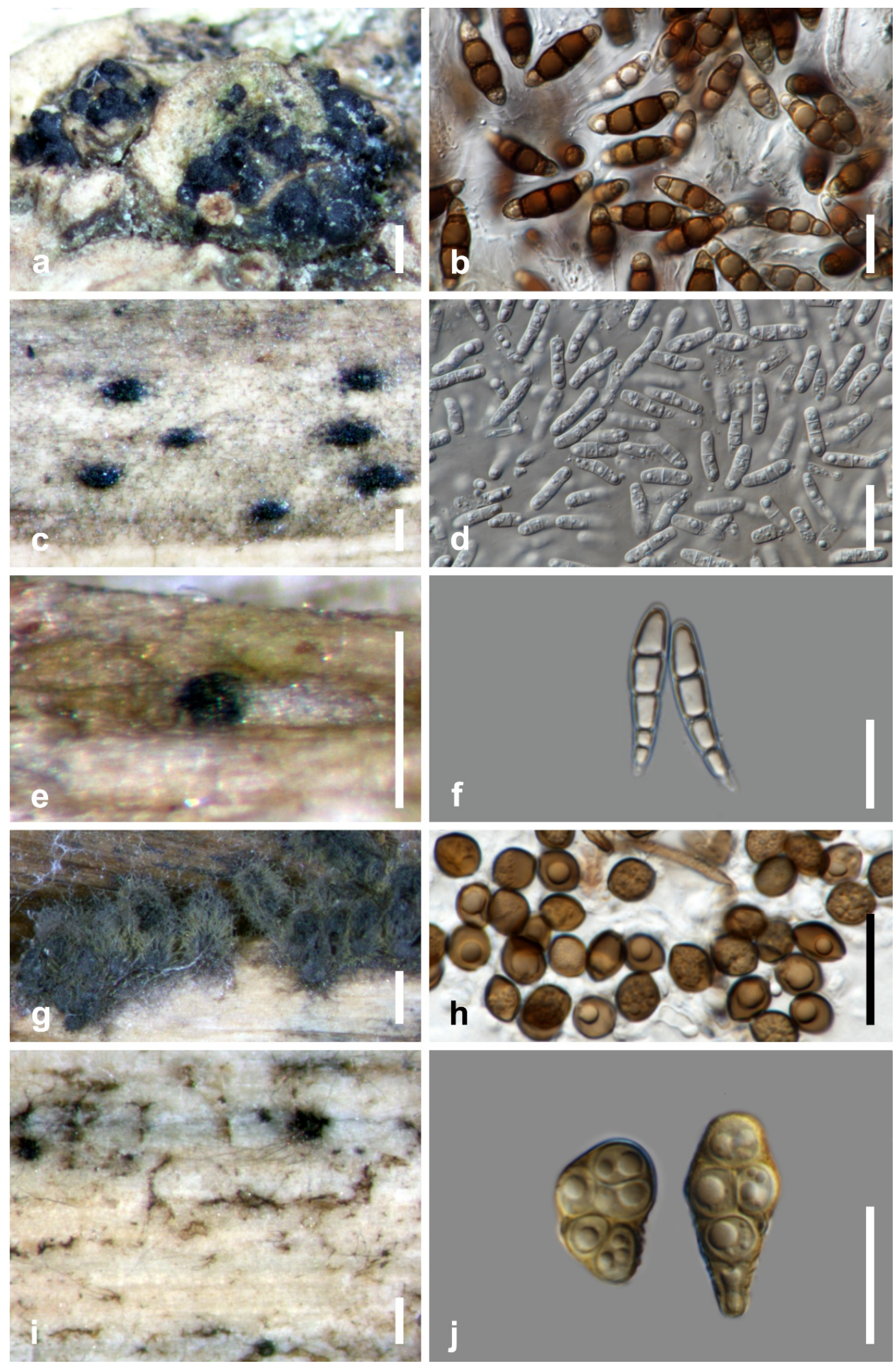

Figure 4. Salt marsh fungi. (a,b) Halobyssothecium obiones from Atriplex portulacoides; (c,d) Halobyssothecium phragmites from culms of Phragmites sp.; (e,f) Buergenerula spartinae from culms of Spartina sp.; (g,h) Chaetomium sp. from stem of Typha sp.; (i,j) Alternaria sp. from culms of Spartina sp. Scale bars: $(\mathbf{a}, \mathbf{g})=500 \mu \mathrm{m} ;(\mathbf{b}, \mathbf{d}, \mathbf{f}, \mathbf{h}, \mathbf{j})=20 \mu \mathrm{m} ;(\mathbf{c}, \mathbf{i})=200 \mu \mathrm{m} ;(\mathbf{e})=100 \mu \mathrm{m}$. 


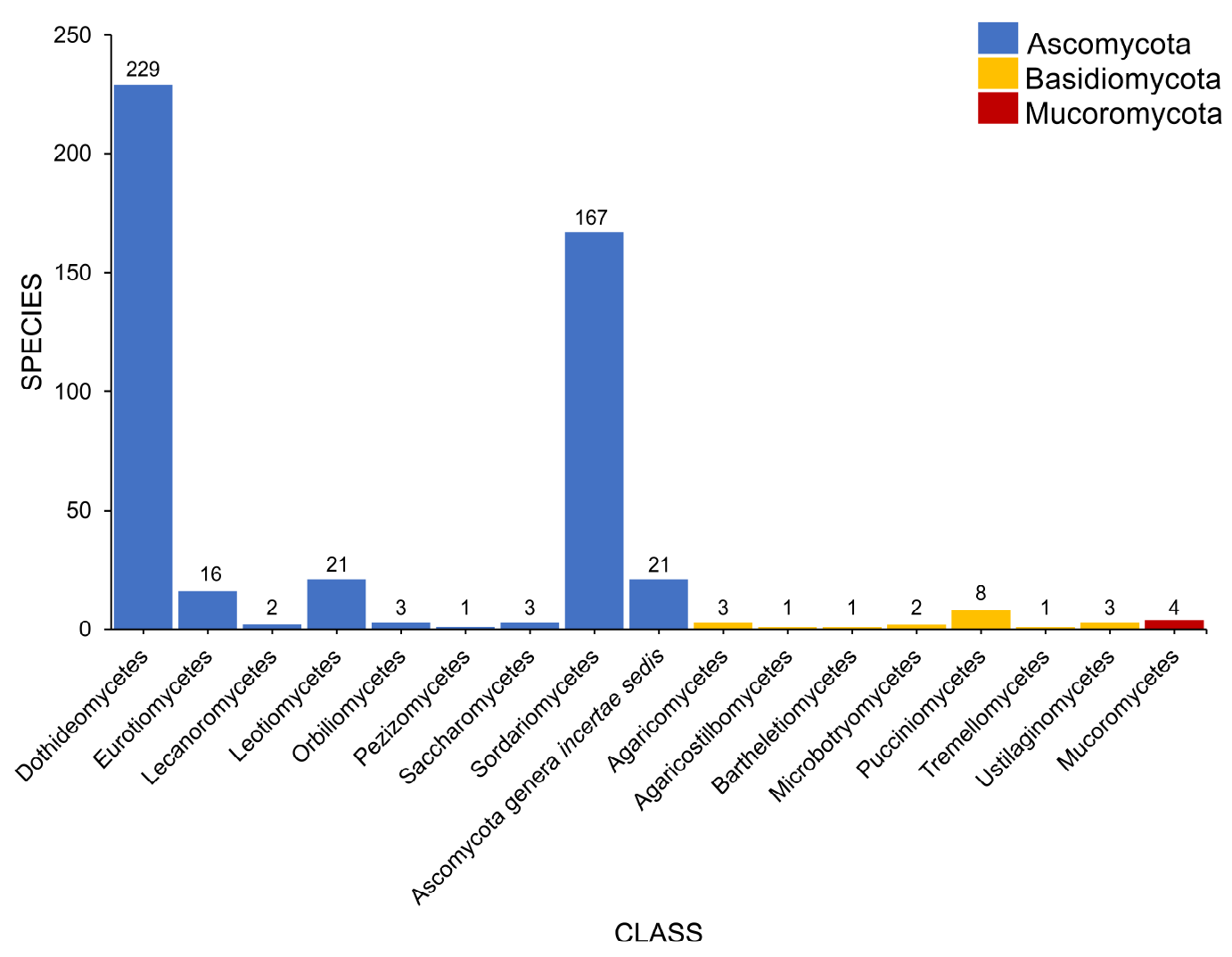

Figure 5. The distribution of salt marsh fungi in different fungal classes.

\subsection{Orders}

Salt marsh fungi recorded from different halophytes were distributed among 48 orders (Table 1, Figure 6). The Pleosporales is the largest order, with 178 taxa recorded followed by Hypocreales (41), Microascales (26), Capnodiales (22), Helotiales (18), Xylariales (17), Sordariales (16), Amphisphaeriales (15), and Eurotiales (13). The remaining 41 orders have less than 10 species (Table 1, Figure 5). Forty-two taxa belong to incertae sedis (Ascomycota genera incertae sedis: 21; Dothideomycetes families incertae sedis: 11; Sordariomycetes families incertae sedis: 9; Xylariomycetidae family incertae sedis: 1).

\subsection{Families}

A total of 108 families and 12 incertae sedis were recorded to be associated with salt marsh fungi (Table 1, Figure 7). Phaeosphaeriaceae and Pleosporaceae account for the largest families with 34 and 31 taxa recorded, respectively. Thirteen families have ten or more than taxa and include Nectriaceae (25), Halosphaeriaceae (25), Didymellaceae (17), Mycosphaerellaceae (14), Lentitheciaceae (13), Massarinaceae (13), Chaetomiaceae (12), Xylariaceae (11), Didymosphaeriaceae (10), Leptosphaeriaceae (10), and Aspergillaceae (10). The remaining 95 families have less than ten species recorded. Forty-four taxa are placed as incertae sedis, wherein 21 of these belong to Ascomycota genera incertae sedis. 


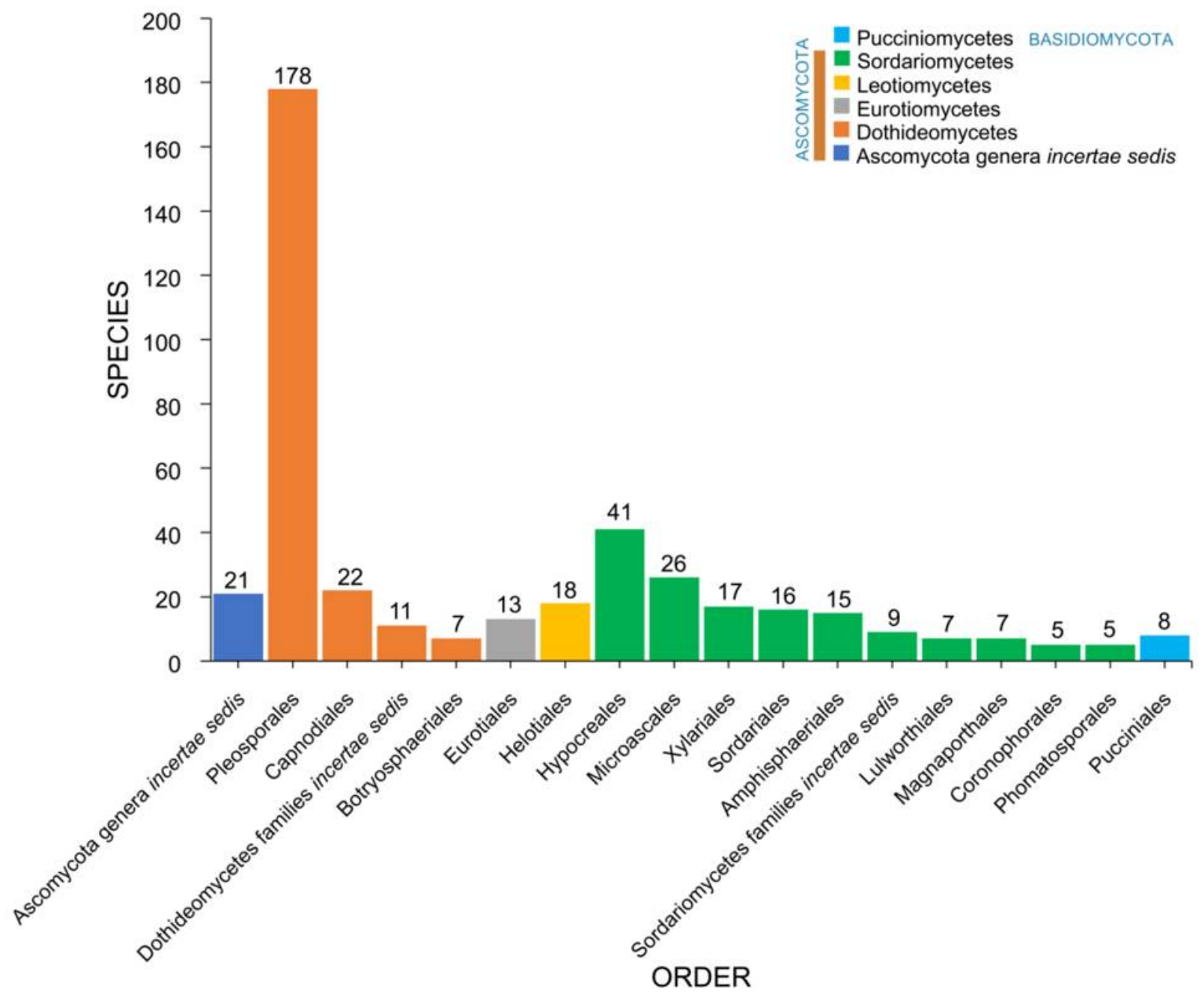

Figure 6. The distribution of salt marsh fungi in major fungal orders.

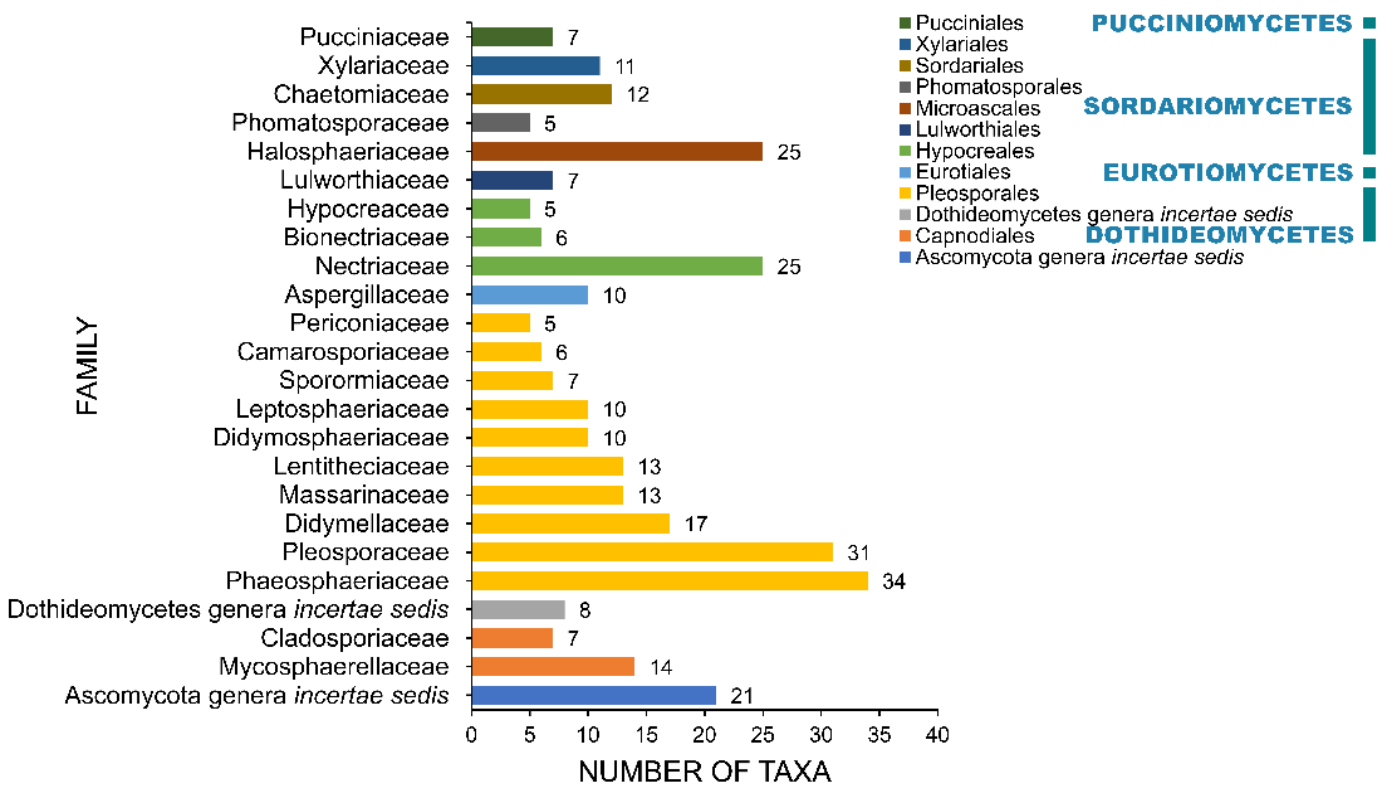

Figure 7. The distribution of salt marsh fungi among major fungal families. 


\section{Diversity of Fungi in Halophytes}

Twenty-seven genera under 11 families (Amaranthaceae, Apiaceae, Caryophyllaceae, Compositae, Juncaceae, Juncaginaceae, Plumbaginaceae, Poaceae, Poaceae, Primulaceae, Ruppiaceae, Typhaceae, Zosteraceae) of halophytes were reviewed for its fungal associates (Table 1, Figure 8). Halophytic species are represented in Figures 1 and 2.

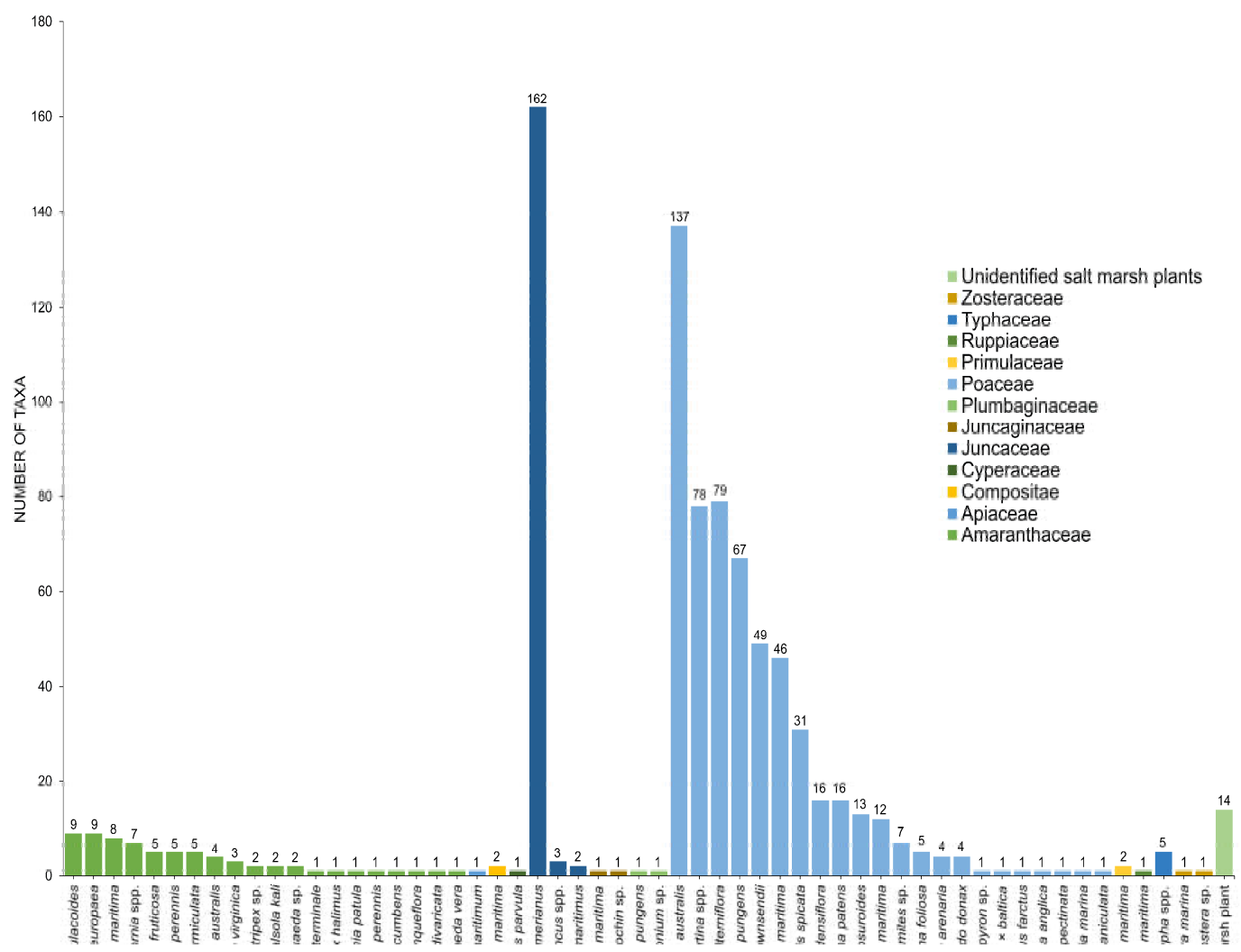

Figure 8. The number of taxa observed from different hosts in salt marsh ecosystems.

\subsection{Amaranthaceae}

Six genera (Arthrocnemum, Atriplex, Salicornia, Salsola, Sarcocornia, Suaeda) represent the Amaranthaceae. Suaeda and Salicornia are the most studied hosts in Amaranthaceae. Ascomycota account for $96.30 \%$ of the 52 taxa recorded in Amaranthaceae (Figure 9, Table 1). Two Pucciniomycetes species, Aecidium suaedae [154] and Uromyces salicorniae [95], represent Basidiomycota. The taxa in Amaranthaceae represent three classes wherein Dothideomycetes accounts for $85.19 \%$ (46 taxa), followed by Sordariomycetes with six taxa reported.

Fungi associated with Suaeda total 18 taxa. Dothideomycetes was represented by 14 taxa $(77.78 \%)$, while three taxa were Sordariomycetes (Cryptovalsa suaedicola [144], Fusarium fujikuroi [62], Moheitospora fruticosae [130]) and one taxon of Pucciniomycetes (Aecidium suaedae [154]).

A total of 14 taxa were documented in Salicornia. Eleven of these belong to Dothideomycetes (Pleosporales: 10; Capnodiales: 1), followed by Sordariomycetes (two taxa: Halocryptovalsa salicorniae [145], Tubercularia pulverulenta [35]), and Pucciniomycetes (one taxon: Uromyces salicorniae [95]).

Fungi from Atriplex total 11 taxa (10 genera) and all of these belong to Pleosporales (Dothideomycetes). Sarcocornia harbors seven taxa (six Dothideomycetes, one Sordariomycetes). Only two taxa (Alternaria spp., Stemphylium spp.) and a single taxon (Mycosphaerella salicorniae) were reported from Salsola [35] and Arthrocnemum [35], respectively. 


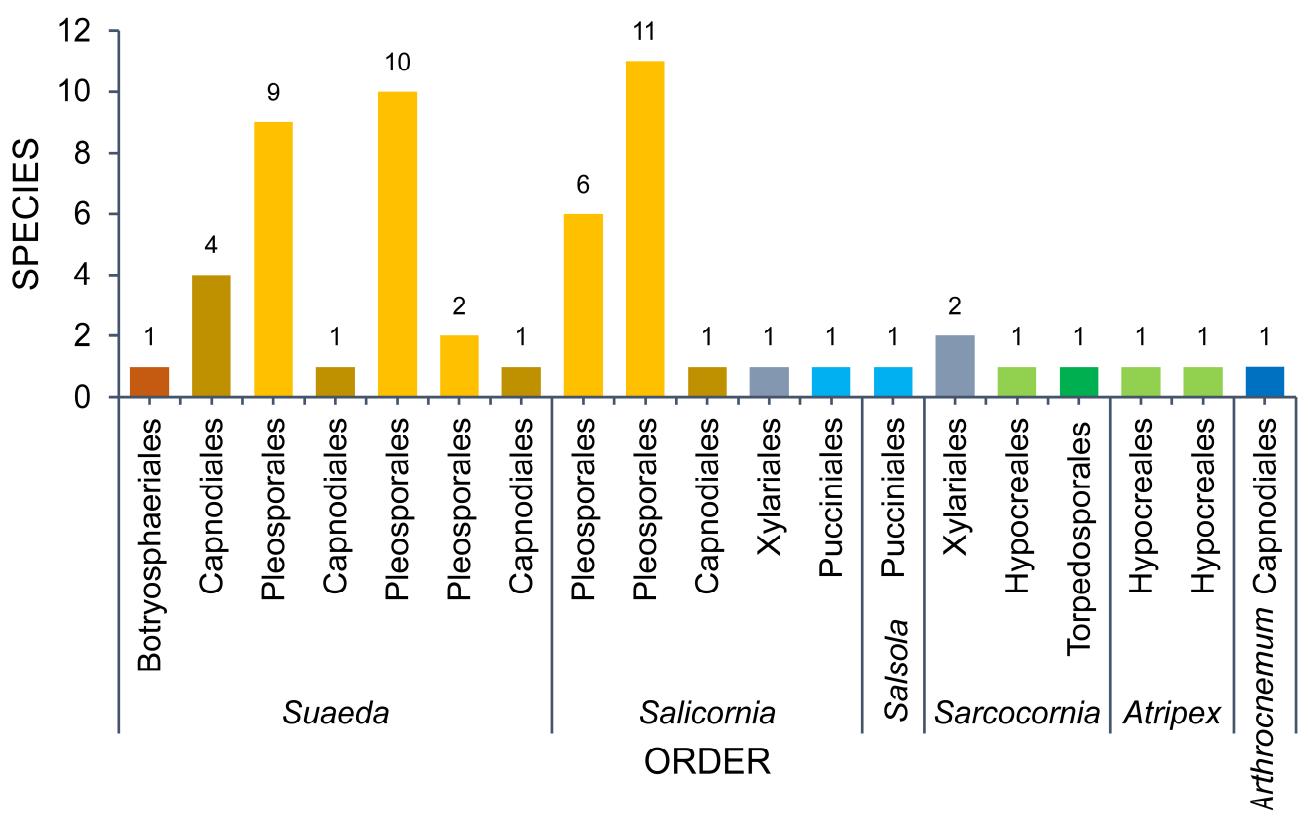

Figure 9. The number of taxa observed from Amaranthaceae.

\subsection{Poaceae}

The association of fungi with grasses have been documented and most of the host plants are members of Poaceae. Ten genera of salt marsh grasses under Poaceae are included in this review wherein Spartina is the most studied of halophytic hosts for direct observation of marine fungi. In addition to Spartina, salt marsh grasses such as Phragmites and Distichlis were well studied also for their fungal associates.

Salt marsh fungi are not well-documented from grasses such as Spartina anglica, $S$. pectinata, Spergularia marina, Uniola paniculata, Elymus farctus, $\times$ Ammocalamagrostis baltica, and Agropyron sp. with one taxon recorded for each host [35]. Furthermore, there are few studies on the fungal composition of Arundo donax (4 taxa) [35] and Ammophila arenaria (four taxa). Marram grass (Ammophila arenaria) is more common in sand dunes and supports quite a diverse fungal community $[157,158]$, while arbuscular mycorrhizal fungi (AMF) play a key role in the establishment, growth, and survival of plants [159].

\subsubsection{Distichlis spicata}

Ascomycota dominates the taxa associated with Distichlis spicata (93.55\%) wherein 16 and 13 species are members of Dothideomycetes and Sordariomycetes, respectively. Pleosporalean taxa constitute the majority of fungi associated with D. spicata (14 species), followed by Hypocreales with nine species recorded. Puccinia distichlidis and Tranzscheliella distichlidis represent the Basidiomycota. A total of 26 genera were recorded as associates of D. spicata and were mostly observed on senescent and decaying leaves.

\subsubsection{Elymus pungens}

Sixty-seven taxa were recorded in Elymus pungens and belong to Ascomycota. Most of the taxa belong to Dothideomycetes (32 taxa), followed by Sordariomycetes (21 taxa), Leotiomycetes, and Eurotiomycetes (6 taxa) (Table 1, Figure 10). 


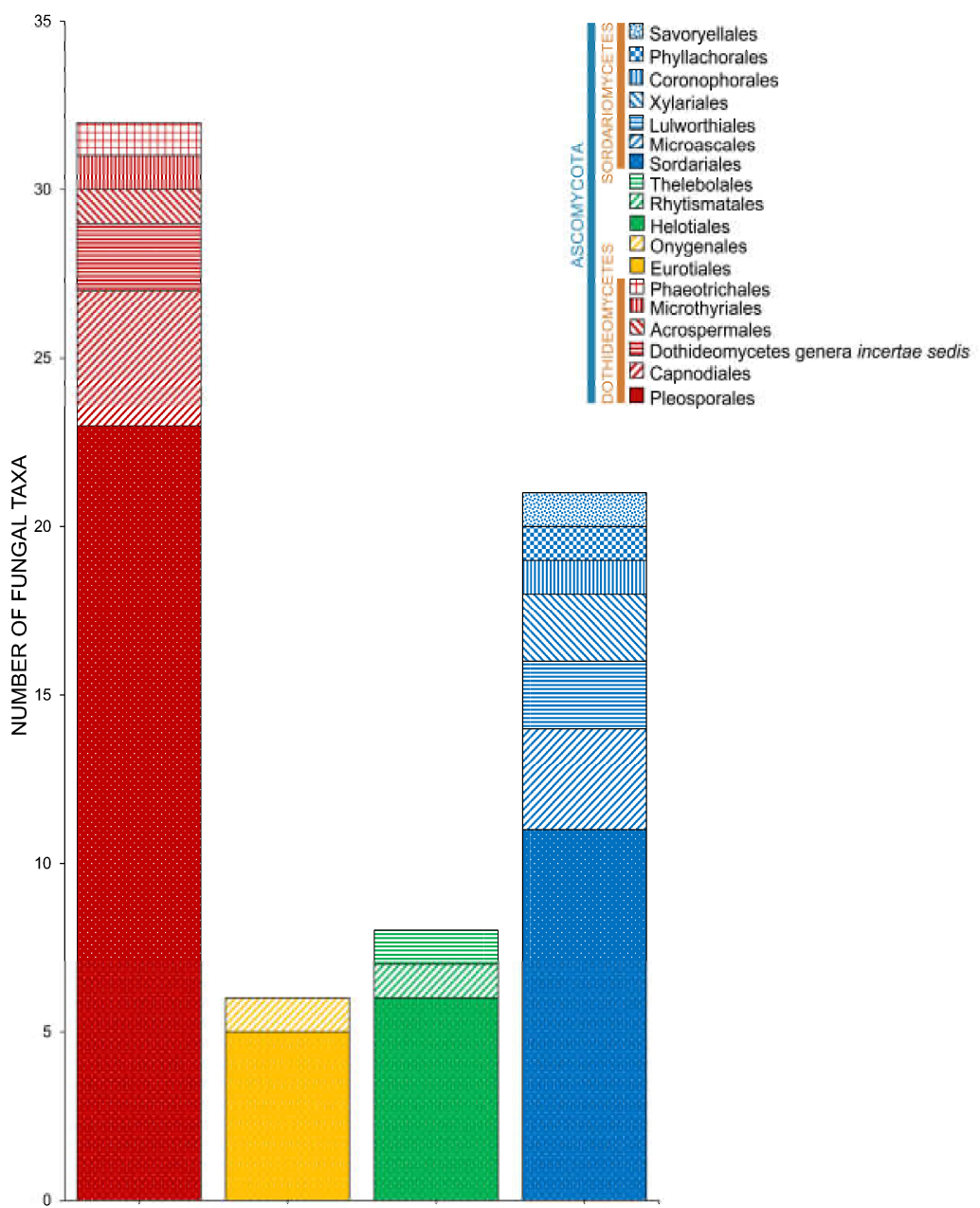

Figure 10. The distribution of fungal taxa associated with Elymus pungens.

\subsubsection{Puccinellia maritima}

A total of 12 taxa (six Sordariomycetes; the following five Dothideomycetes: Micronectriella agropyri, Lautitia danica, Leptosphaeria pelagica, Septoriella vagans, Paradendryphiella salina; one Leotiomycetes: Thelebolus crustaceus) were recorded in Puccinellia maritima [38]. All the taxa from Sordariomycetes belong to Sordariales (Chaetomium elatum, C. globosum, C. thermophilum, Corynascus sepedonium, Thermothielavioides terrestris, Sordaria fimicola) [38].

\subsubsection{Spartina}

A total of 149 taxa (141 Ascomycota, 6 Basidiomycota, 2 Mucoromycota) were recorded in Spartina. The majority of the taxa belong to Dothideomycetes (70 taxa), followed by Sordariomycetes (59 taxa). Pleosporaceae and Halosphaeriaceae dominate the fungi documented in Spartina with 19 and 17 taxa recorded, respectively. Spartina alterniflora, S. maritima, and Spartina $\times$ townsendii harbor 79, 46, and 49 taxa, respectively (Figure 11, Table 1). A total of 78 taxa were recorded in the unidentified Spartina species. The identification of the Spartina species can be challenging, wherein species are morphologically similar. 


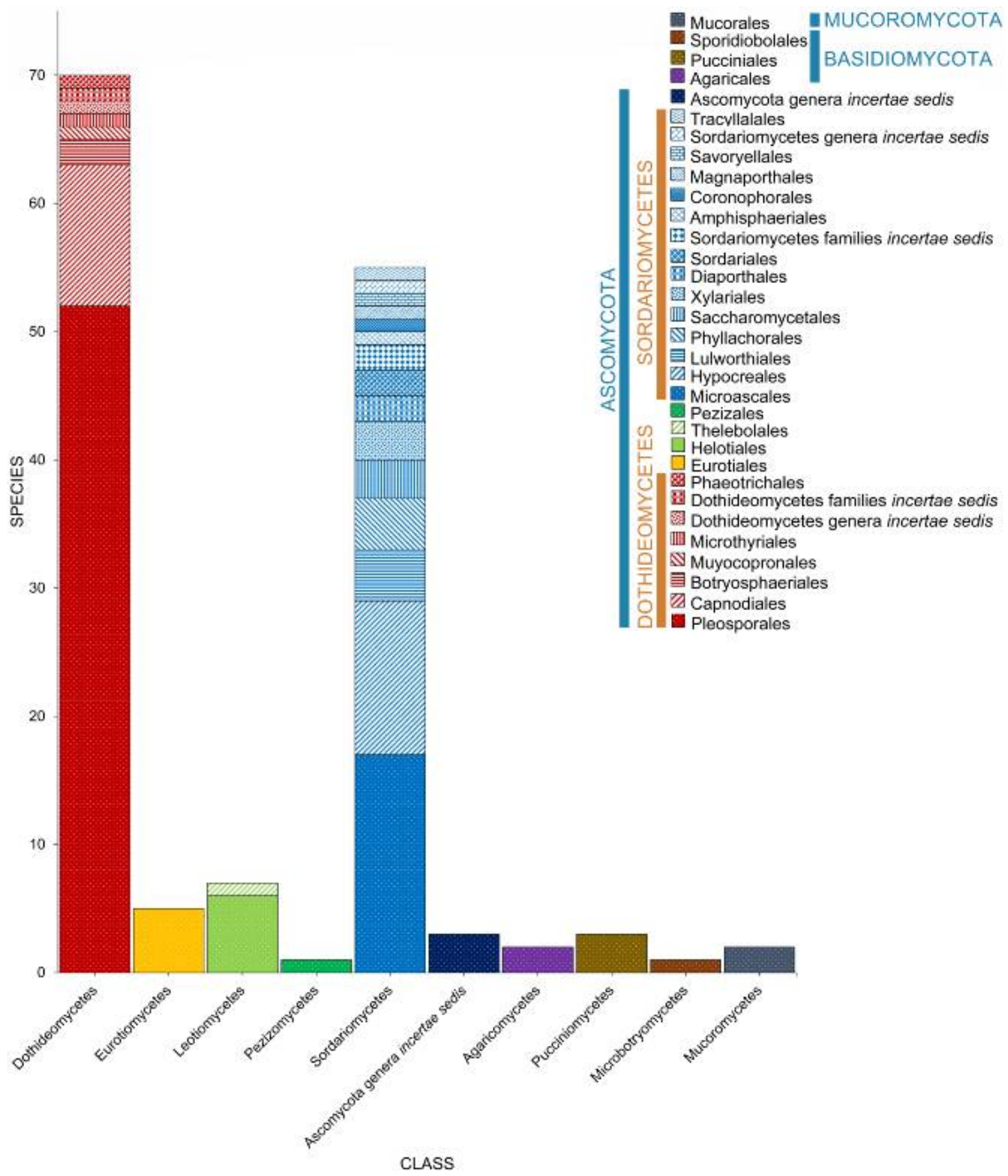

Figure 11. The distribution of fungal taxa associated with Spartina.

Halobyssothecium obiones was recorded from six species of Spartina (S. alterniflora [20,35, $52,61,71,74,80-82]$, S. cynosuroides [35], S. densiflora [64], S. maritima [31,54,59,63], S. patens [36], S. townsendii $[49,65]$, and the unidentified Spartina sp. $[32,35,36,58,84])$, while six Spartina spp. harbors unidentified Mycosphaerella species. Six species (Leptosphaeria pelagica, Lulworthia spp., Phaeosphaeria halima, Phaeosphaeria spartinicola, Phoma spp., Stagonospora spp.) were recorded in five different hosts. The unidentified Spartina species harbors 28 unique species. Amongst the taxa found in Spartina, 32 species can only be found in $S$. alterniflora, while $S$. maritima harbors 21 unique species, the most intensively surveyed species. 


\subsubsection{Phragmites}

A total of 138 taxa have been documented in Phragmites (Figure 12, Table 1). Most of the taxa belong to Ascomycota (131 taxa), while six taxa represent the Basidiomycota. Dothideomycetes dominates half of the taxa in Phragmites (71 taxa, $51.45 \%$ ) followed by Sordariomycetes (44 taxa, 31.88\%), Leotiomycetes (6 taxa, 4.35\%), Ascomycota genera incertae sedis (5 taxa, 3.62\%), Eurotiomycetes (3 taxa, 2.17\%), Orbiliomycetes ( 2 taxon, $1.45 \%$ ), and Pucciniomycetes (1 taxa, $1.45 \%$ ). One taxon each were recorded to Agaricomycetes [40], Bartheletiomycetes [41], Lecanoromycetes [39], Microbotryomycetes [39,50], and Tremellomycetes $[39,40]$. Pleosporalean taxa accounts for the highest number of fungi associated with Phragmites (42.75\%, 59 taxa).

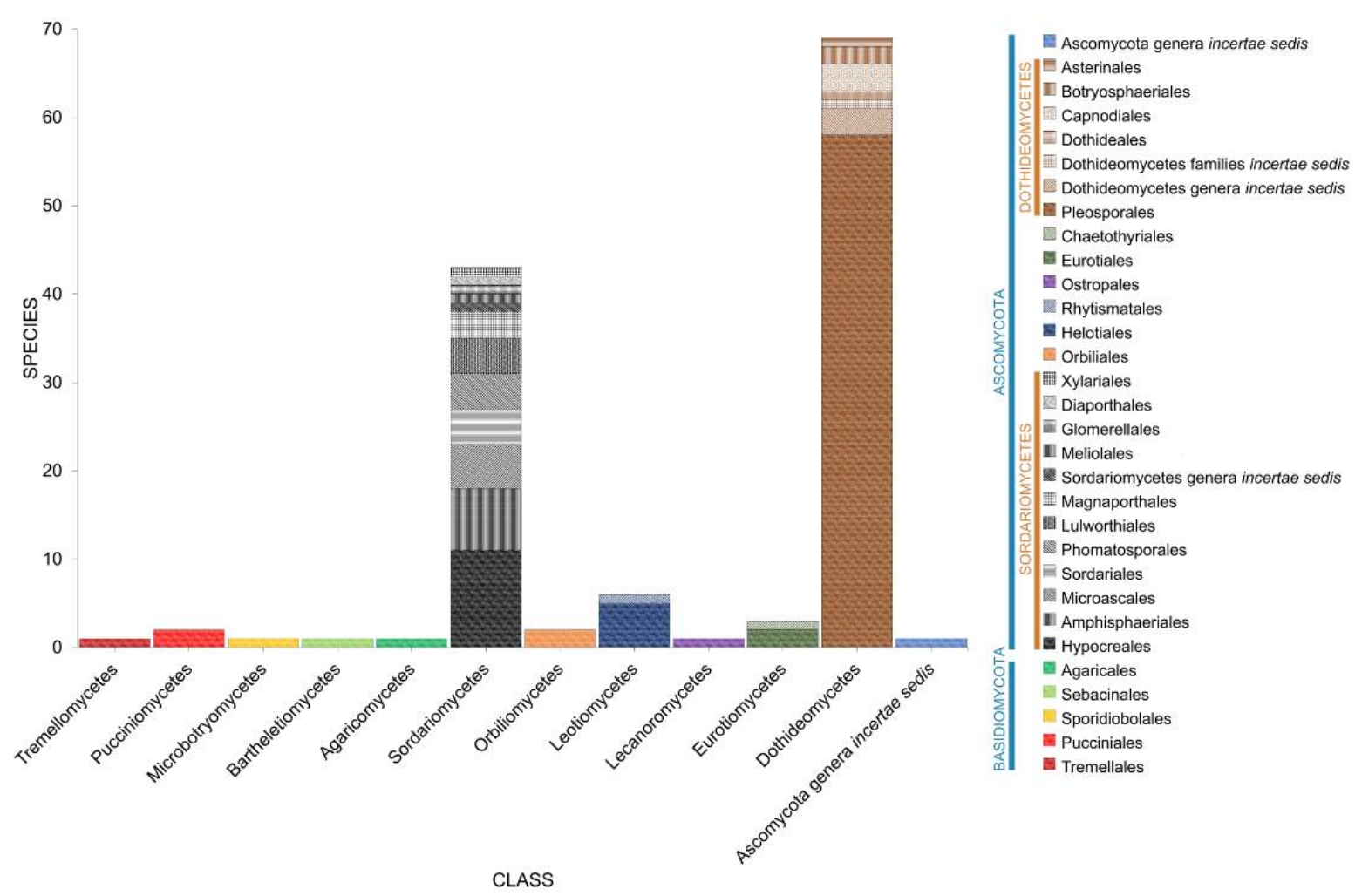

Figure 12. The distribution of fungal taxa associated with Phragmites.

Phragmites australis harbors diverse fungi that totals to 137 taxa (101 genera) [39-41,50,79,115]. Seven species (Arthrinium arundinis [62], Halazoon fuscus [87], Halobyssothecium phragmitis [85], Keissleriella linearis [85], Phomatospora dinemasporium [62], Remispora hamata [87], Setoseptoria phragmitis [87]) were recorded in unidentified Phragmites species.

\subsection{Juncaceae}

Juncus roemerianus, J. maritimus, and an unidentified Juncus species represent Juncaceae. Salt marsh fungi are diverse in Juncus and dominated by Ascomycota, which constitutes $97.58 \%$ of the 165 reported taxa (Figure 13, Table 1). Stilbum sp. represented the Basidiomycota, while three taxa (Blakeslea trispora, Mucor sp., Syncephalastrum racemosum) of Mucoromycota were recorded. Dothideomycetes and Sordariomycetes account for the highest number of Juncus-associated fungi with 72 (43.64\%) and 64 (38.79\%) taxa documented. 


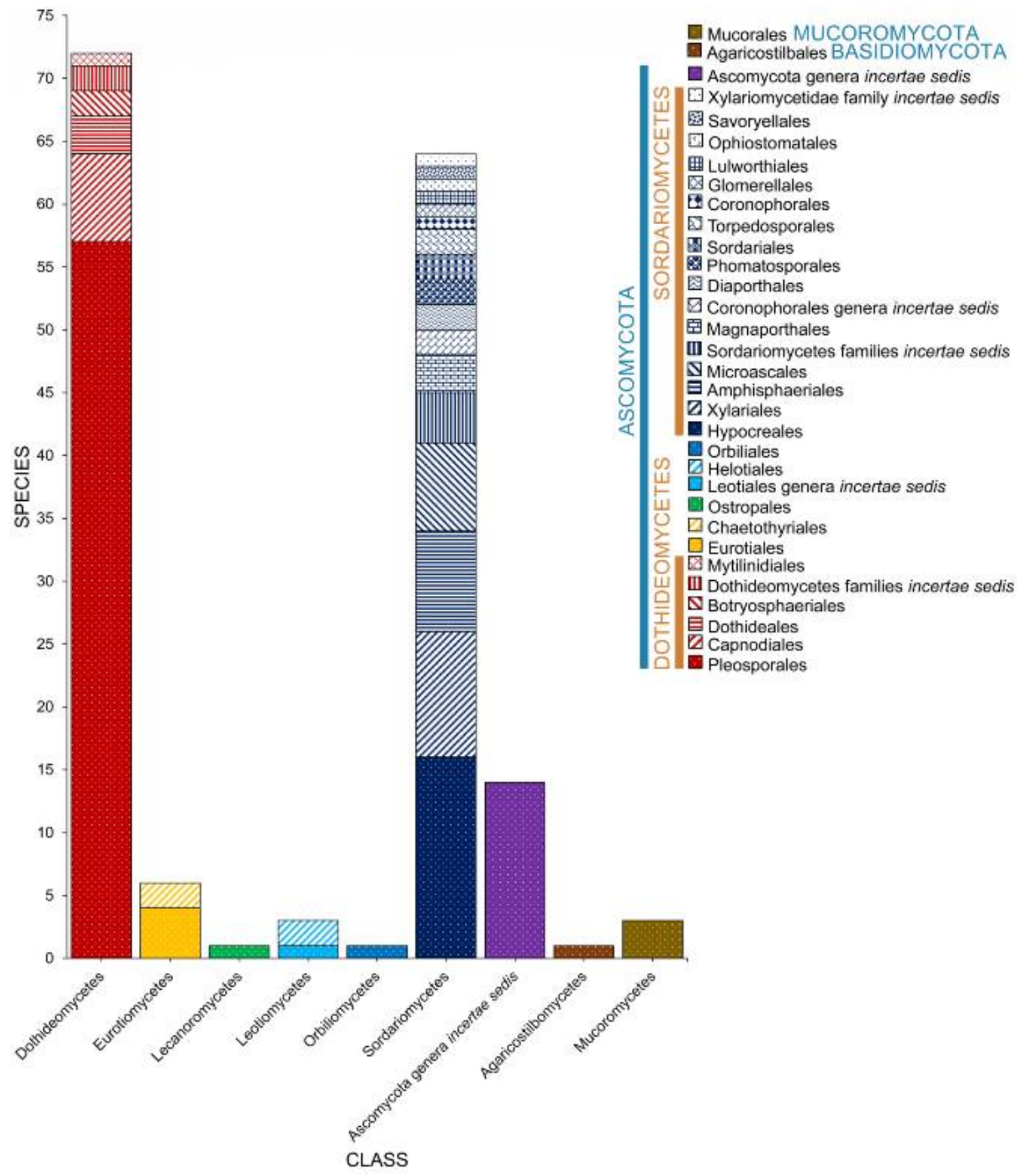

Figure 13. The distribution of fungal taxa associated with Juncus.

Juncus roemerianus has been extensively studied for its associates with 162 documented taxa $[32,42,43,60,66,76-78,97,98,104,105,110,116-118,135,147,148]$. Few species were reported to Juncus maritimus that harbor only two taxa (Leptosphaeria albopunctata, Phaeosphaeria neomaritima) [35]. Phaeosphaeria neomaritima [36,52,71,80], P. spartinicola [52], and Monodictys pelagica [35] were observed in an unidentified species of Juncus.

Phragmites australis harbors diverse fungi that totals to 137 taxa (101 genera) [39-41,50,79,115]. Seven species (Arthrinium arundinis [62], Halazoon fuscus [87], Halobyssothecium phragmitis [85], Keissleriella linearis [85], Phomatospora dinemasporium [62], Remispora hamata [87], Setoseptoria phragmitis [87]) were recorded in unidentified Phragmites species. 


\subsection{Other Families}

Few reports on salt marsh fungi are from the following hosts: Apiaceae: Crithmum maritimum (one taxon: Phoma sp.), Typhaceae: Typha spp. (five taxa: Arundellina typhae, Chaetomium sp., Magnisphaera spartinae, Pleospora pelagica, Remispora hamata); Compositae: Artemisia maritima (two taxon: Neocamarosporium artemisiae, N. maritimae); Caryophyllaceae: Spergularia marina (one taxon: Cladosporium algarum); Plumbaginaceae: Limonium sp. (one taxon: Mycosphaerella salicorniae); Armeria pungens (one taxon: Mycosphaerella staticicola); Juncaginaceae: Triglochin sp. and T. maritima (one taxon: Stemphylium triglochinicola); Primulaceae: Lysimachia maritima (two taxa: Leptosphaeria orae-maris, Stemphylium vesicarium); Ruppiaceae: Ruppia maritima (one taxon: Flamingomyces ruppiae); and Zosteraceae: Zostera marina (one taxon: Corollospora ramulosa) and Zostera sp. (Asteromyces cruciatus). Alva et al. [160] report Penicillium chrysogenum as an endophyte from Zostera japonica.

Fourteen taxa were documented from unidentified salt marsh plants. All of the taxa belong to Ascomycota (seven Dothideomycetes, five Sordariomycetes, one Eurotiomycetes). Pleosporalean taxa from six families account for half of the taxa (the following seven species: Camarosporium palliatum, C. roumeguerei, Coniothyrium obiones, Halobyssothecium obiones, Periconia sp., Loratospora aestuarii, Pleospora pelvetiae).

\section{Geographical Distribution of Salt Marsh Fungi}

The salt marsh fungi reported are from countries of three major oceans, as documented in Figure 14. The Atlantic Ocean consists of 12 countries, wherein the USA had the highest number of species recorded (232 taxa) followed by the UK (101 taxa), the Netherlands (74 taxa), and Argentina (51 taxa). China had the highest number of salt marsh fungi in the Pacific Ocean with 165 taxa reported, while in the Indian Ocean, India reported the highest taxa (16 taxa). Most of the biodiversity studies documenting salt marsh fungi in the Atlantic Ocean are mostly from the USA and the UK and this reflects the high number of taxa $[32,36,38,49,61]$. China ranked second with the most number of salt marsh fungal taxa, mainly due to the biodiversity study in Phragmites australis conducted by Poon et al. [41].

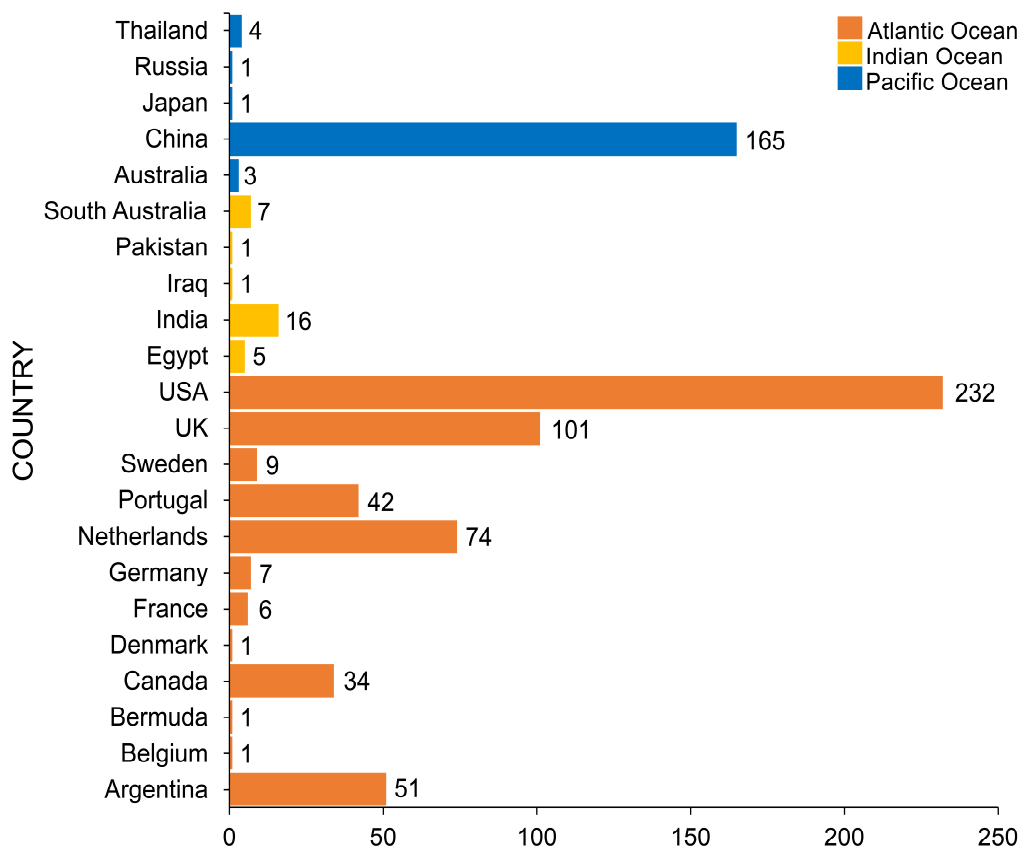

Figure 14. The number of salt marsh fungi reported in the Pacific, Atlantic, and Indian Oceans.

The geographical distribution of salt marsh fungi and the different halophytes are presented in Figure 15. The fungi associated with salt marsh grass Phragmites australis have been studied in different countries (Australia, Belgium, Egypt, France, Germany, China, Iraq, Japan, the Netherlands, South Australia, Thailand). Spartina alterniflora was recorded 
in countries along the Atlantic (Argentina, Canada, France, USA) and the Indian Ocean (India), but lacks data from countries in the Pacific Ocean.

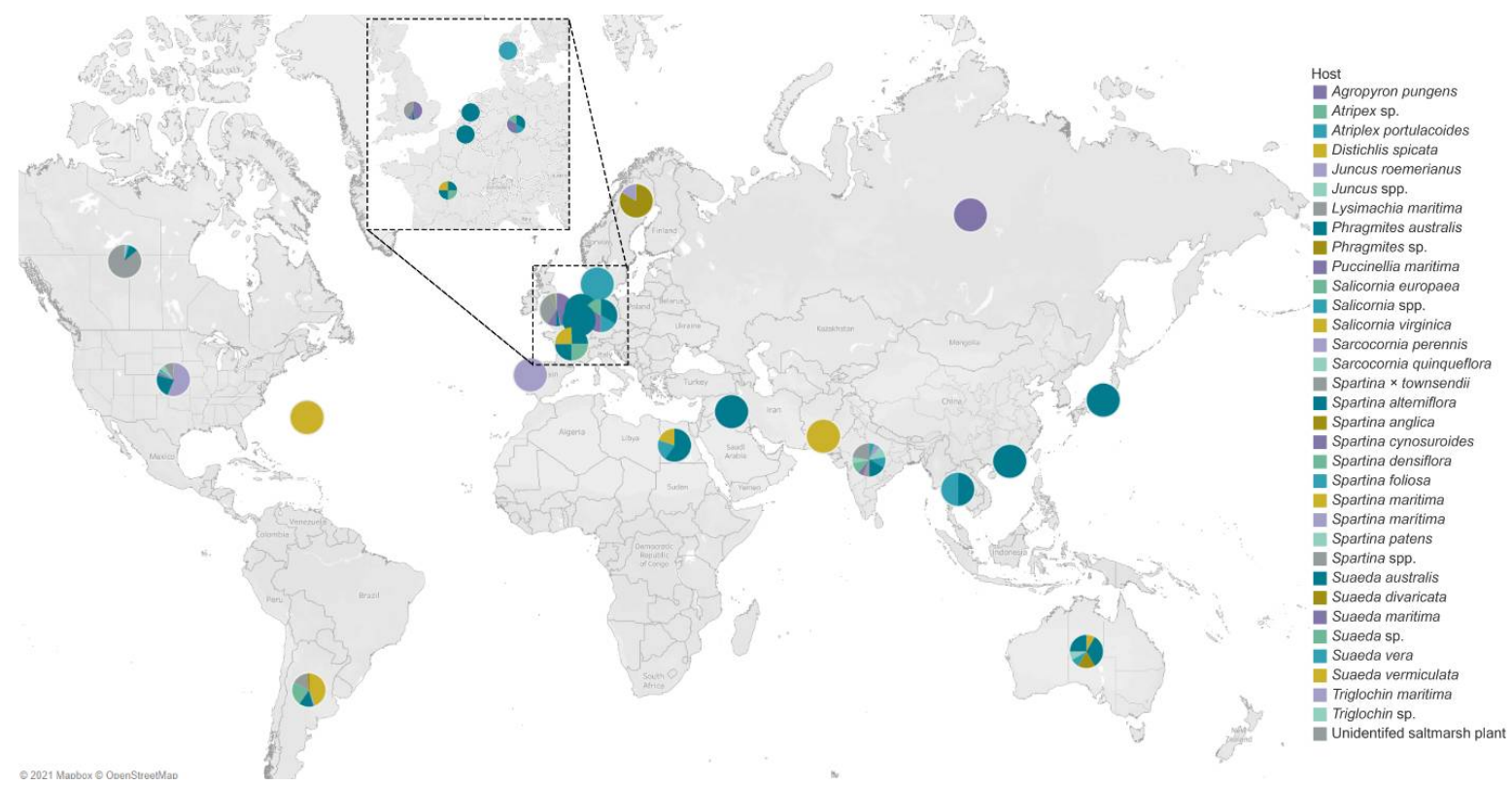

Figure 15. Map of countries showing the global distribution of fungal diversity studies in halophytes. The different color of each pie chart represents the hosts, and the angle measured the number of their fungal associates.

\section{United States of America}

Most of the studies of halophytes-associated fungi were concentrated on the United States of America (USA) (Figure 16). Table 1 lists the salt marsh fungi in 20 states. Florida has been the frequently studied, wherein seven hosts (Juncus roemerianus: 108 taxa; Spartina $\times$ townsendii: 1; Spartina alterniflora: 16; Spartina cynosuroides: 3; Spartina densiflora: 1; Spartina patens: 2; Spartina spp.: 3) were observed for salt marsh fungi. Six hosts were studied in North Carolina, wherein Juncus roemerianus harbored the highest number of fungi (48 taxa). In Rhode Island, Spartina alterniflora accounts for the highest number of fungi, with 41 taxa recorded.

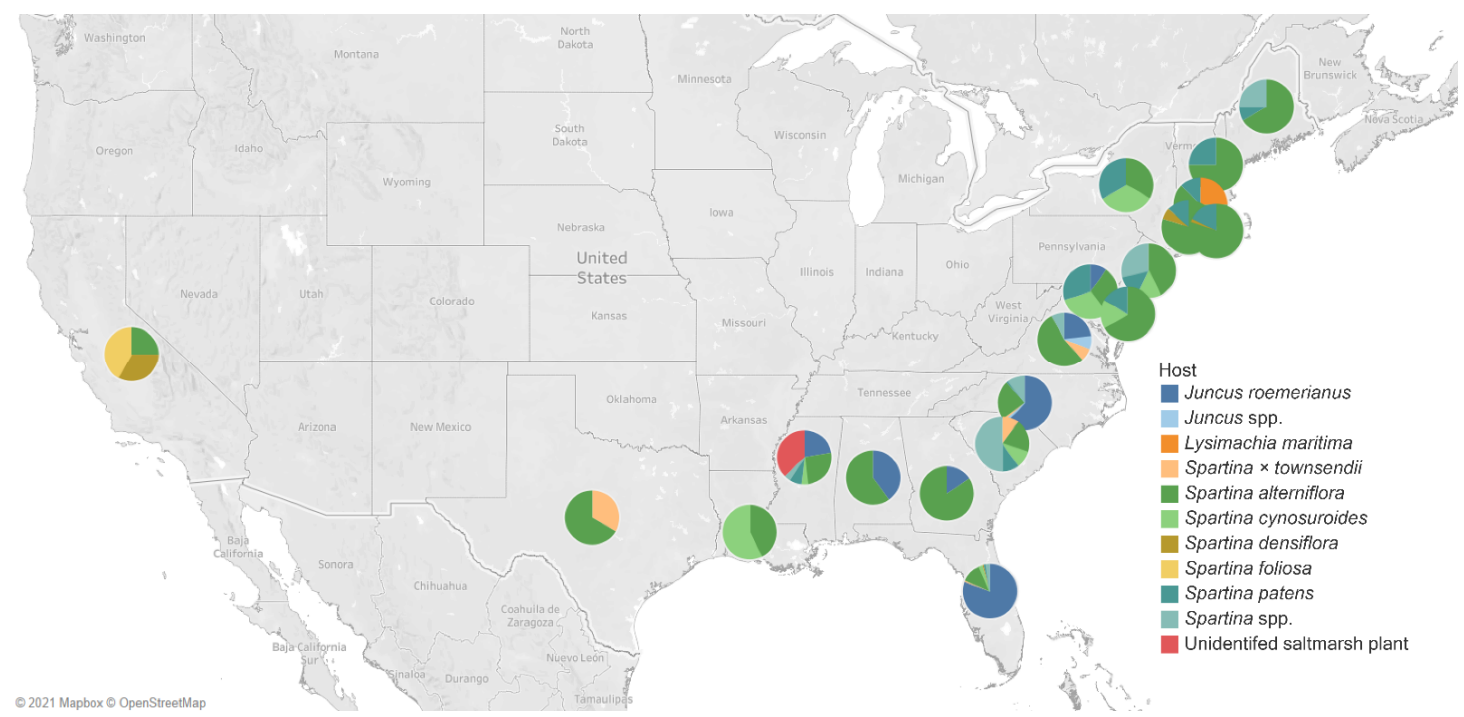

Figure 16. Map of the United States of America (USA) showing the distribution of fungal diversity studies of halophytes in different states. The different color of each pie chart represents the hosts, and the angle measured the number of their fungal associates. 


\section{Conclusions and Future Perspectives}

Most studies of fungi on salt marsh plants are from Spartina, Juncus, and Phragmites, probably due to the huge biomass generated by these taxa. The mycota of less bulky halophytes (e.g., Limonium, Triglochin, Uniola) and litter from the surrounding sea grass beds washed off to marsh areas (e.g., Zostera japonica, Z. marina, Z. noltii) are also less represented, or these hosts are yet to be explored. The checklist presented in the current study updates the list of Calado and Barata [34] and the inclusion of fungi associated with rarely studied halophytes record 486 taxa worldwide. Ascomycota dominate the taxa (463 taxa) and are comprised mostly of Dothideomycetes with their ability to eject their ascospores forcibly and widely, spore type, the formation of ascomata or ascostromata under a clypeus or just immersed in thin leaves, and an ability to decompose lignocellulose substrates [57,161]. Meyers et al. [162] showed that salt marsh yeasts and the ascomycete, Buergenerula spartinae, produce degradative enzymes and utilize simple carbon and nitrogen compounds. The yeast, Pichia spartinae, produces $\beta$-glucosidase and other degradative enzymes. Gessner [74] demonstrated that a number of salt marsh fungi isolated from Spartina alterniflora, Zostera sp., and Z. marina produced enzymes capable of degrading cellulose, cellobiose, lipids, pectin, starch, tannic acid, and xylan and, thus, play a key role in the degradation of storage and structural compounds. Salt marsh fungi might possess high biotransformation and metabolic abilities, which could be related to their ecology.

Basidiomycota (19 taxa) and Mucoromycota (4 taxa) are poorly represented in salt marsh ecosystems as they are in other marine habitats [163]. There are no records of Chytridiomycota listed in the present work and only a few authors detected this group, and other basal fungal lineages, in salt marsh ecosystems using molecular analysis [164-167]. These groups are worth exploring to determine the overall fungal communities in the salt marsh ecosystems. Many chytrids and other basal fungi are more challenging to cultivate and require different isolation methods (e.g., baiting techniques in liquid culture) than the saprobes, methods that have rarely been applied in the study of saltmarsh plants. When appropriate techniques are used, chytrids and other zoosporic organisms have been reported. For example, the fungal-like organism Phytophthora inundata has been recovered from the halophilic plants Aster tripolium and Salicornia europaea, while P. gemini and $P$. chesapeakensis occur on Zostera marina, and Salisapilia nakagirii on the decaying litter of Spartina alterniflora (www.marinefungi.org; accessed on 10 May 2021, [163]). Marine chytrids have been isolated from substrates such as seaweeds and mangrove leaves [163].

The taxa listed are mostly saprobes and these can be attributed to the inclusion of salt marsh fungi observed directly from the different host parts, which are mostly submerged decaying substrates. When compared to saprobic fungi in halophytes, few studies have been carried out on the diversity of endophytes and pathogens and their interaction in the salt marsh ecosystems. Surveys on endophytic fungi from halophytes using cultivation-dependent methods coupled with molecular approaches, showed that endophytes were dominated by Ascomycota and a few belonged to Basidiomycota and Zygomycota [168-175]. Pathogenic fungi from salt marsh ecosystems are poorly documented but play a significant role in the dynamics of the ecosystem [176-178]. For example, Govers et al. [179] reported that the fungal-like organisms Phytophthora gemini and P. inundata caused widespread infection of the common seagrass species, Zostera marina (eelgrass), across the northern Atlantic and Mediterranean that threatened the conservation and restoration of vegetated marine coastal systems. Likewise, Claviceps purpurea affects the viability of Spartina townsedii in south coast UK salt marshes. Fisher et al. [180] noted that $\mathrm{Cl}$. purpea in the Alabama and Mississippi coastlines rendered the seeds of one of the primary salt marsh grasses sterile. Raybold et al. [181] recorded epidemics of C. purpurea on Spartina anglica in Poole Harbor (UK) and that ergot growth was detrimental to seed production. These underexplored fungal groups are worthy to be explored for their ecological and biotechnological importance.

This shows how salt marsh fungal studies were concentrated in countries in the Atlantic Ocean specifically the USA (232 taxa) and the UK (101 taxa). Many salt marsh areas remain 
unexplored, especially those in the Indian and Pacific Oceans, and these areas are hotspots of biodiversity and novel fungal taxa based on the exploration of various habitats [85,100,163,182-187]. Recently, novel species were isolated in halophytes $[85,100,145]$ and further taxa remain to be discovered, isolated, and sequenced, while vast areas worldwide have yet to be surveyed. For example, salt marsh plants are immensely numerous, diverse, and common along the south-east coast of Australia, yet little is known of their fungal associates [188].

The salt marsh vegetation and its fungal associates are adapted to salt stress and inundation and are subjected to extreme environmental conditions such as being periodically wet to different lengths of time leading to drying out at low tides and exposure to high temperatures and drying out at midday. Many are well adapted to prevailing conditions by their fleshy leaves (Suaeda australis), others can tolerate high flooding.

Few data are currently available on the specificity of fungi on their salt marsh hosts. Figure 17 shows the number of fungal taxa recorded from the three commonly studied hosts, Juncus, Phragmites, and Spartina, wherein there is little overlap in the species composition. One of the common species on Spartina plants is undoubtedly Halobyssothecium obiones, while Leptosphaeria pelagica is common. A common ascomycete on Atriplex portulacoides and Suaeda maritima is Decorospora gaudefroyi. Host plants that have been little surveyed for fungi are Limonium vulgare (sea lavender) and Atriplex portulacoides (sea purslane), yet they do support a number of taxa, e.g., Neocamarosporium obiones and Amarenomyces ammophilae. The fungal community reported on Juncus roemerianus in the salt marsh at North Carolina is significantly different from those on Spartina and Phragmites. It remains to be seen if this is due to the host plant or its geographical location.

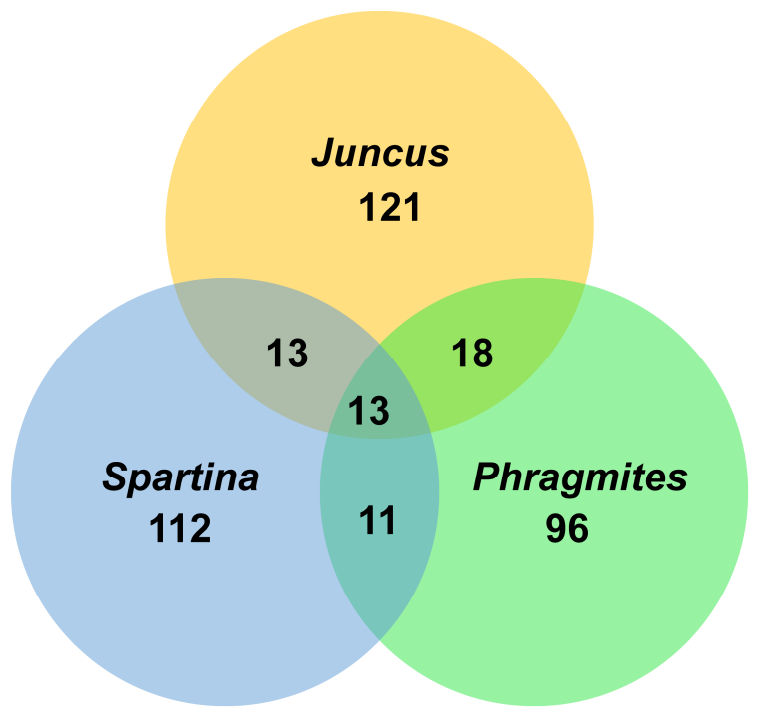

Figure 17. Venn diagram showing the association of salt marsh fungi from commonly studied halophytes.

Another groups of fungi that have not been fully studied in the salt marsh habitat are yeasts, as these also require specific techniques for their isolation from the water column or from plant tissue. Spencer et al. [189] recovered a number of yeasts from the vicinity of Spartina townsendii, as follows: very numerous Cryptococcus spp.; Trichosporon cutaneum; Trichosporon pullulans; the relatively rare species, Metschnikowia bicuspidata and Cryptococcus flavus; and Saturnospora ahearnii [190]. Although marine yeasts are common in sea water and deep seawater vents [163], their large-scale sampling in salt marshes remains a challenge for the future.

Currently, the salt marsh ecosystem has been threatened both by global warming and human activity. Sea-level rises brought about by climate change alter the location and character of the land-sea interface wherein salt marsh vegetation moves upward and inland. The increase in the sea level may not lead to the loss of coastal marshes, but the resiliency will depend on the ability of halophytes to migrate upland. Susceptible 
areas are organogenic marshes and areas where sediment is limited, potentially leading to catastrophic shifts and marsh loss. In this paper, a total of 57 plant taxa under 27 genera were reviewed for their fungal associates. The halophytes included here are only approximately $11 \%$ of the total number of species of salt marsh plants worldwide. Thus, many salt marsh fungi await discovery with wider host plant sampling and the use of a wider range techniques for their isolation. For this reason, it is imperative to study the halophytic fungi to document not just biodiversity but also to discover novel taxa restricted only to this kind of habitat.

Author Contributions: Conceptualization: M.S.C., K.D.H. and E.B.G.J.; methodology: M.S.C., K.D.H. and E.B.G.J.; formal analysis and investigation: M.S.C., K.D.H. and E.B.G.J.; resources: K.D.H. and E.B.G.J.; writing—original draft preparation, M.S.C.; writing—review and editing, K.D.H., E.B.G.J. and I.P.; supervision, K.D.H. and E.B.G.J.; funding acquisition, K.D.H. and E.B.G.J. All authors have read and agreed to the published version of the manuscript.

Funding: K.D.H. thanks the Thailand Research Fund for the grant entitled "Impact of climate change on fungal diversity and biogeography in the Greater Mekong Subregion" (Grant No. RDG6130001). E.B.G.J. is supported under the Distinguished Scientist Fellowship Program (DSFP), King Saud University, Kingdom of Saudi Arabia.

Institutional Review Board Statement: Not applicable.

Informed Consent Statement: Not applicable.

Data Availability Statement: Not applicable.

Acknowledgments: M.S.C. is grateful to the Mushroom Research Foundation and the Department of Science and Technology—Science Education Institute (Philippines). K.D.H. thanks Chiang Mai University.

Conflicts of Interest: The authors declare no conflict of interest.

\section{References}

1. Bertness, M.D. Atlantic Shorelines: Natural History and Ecology; Princeton University Press: Princeton, NJ, USA, 2008.

2. Öztürk, M.; Altay, V.; Altundă̆, E.; Gücel, S. Halophytic plant diversity of unique habitats in Turkey: Salt mine caves of Çankırı and Iğdır. In Halophytes for Food Security in Dry Lands; Khan, M.A., Ozturk, M., Gul, B., Ahmed, M.Z., Eds.; Academic Press: New York, NY, USA, 2016; pp. 291-315.

3. Adam, P. The saltmarsh biota. In Saltmarsh Ecology; Adam, P., Ed.; Cambridge University Press: Cambridge, UK, $2011 ;$ pp. 72-145.

4. Macdonald, K.B. Plant and animal communities of Pacific North American salt marshes. In Wet Coastal Formations; Chapman, V.J., Ed.; Elsevier: Amsterdam, The Netherlands, 1976; pp. 167-191.

5. Saenger, P.; Specht, M.M.; Specht, R.L.; Chapman, V.J. Mangal and coastal salt-marsh communities in Australasia. In Wet Coastal Ecosystems; Chapman, V.J., Ed.; Elsevier: Amsterdam, The Netherlands, 1977; pp. 293-345.

6. Macdonald, K.B. Coastal salt marsh. In Terrestrial Vegetation of California; Barbour, M.G., Major, J., Eds.; Wiley: New York, NY, USA, 1988; pp. 263-294.

7. Costanza, R.; D’Arge, R.; De Groot, R.; Farber, S.; Grasso, M.; Hannon, B.; Limburg, K.; Naeem, S.; O’Neill, R.V.; Paruelo, J.; et al. The value of the world's ecosystem services and natural capital. Nature 1997, 387, 253-260. [CrossRef]

8. Mcowen, C.J.; Weatherdon, L.V.; Van Bochove, J.W.; Sullivan, E.; Blyth, S.; Zockler, C.; Stanwell-Smith, D.; Kingston, N.; Martin, C.S.; Spalding, M.; et al. A global map of saltmarshes. Biodivers. Data J. 2017, 5, 11764. [CrossRef]

9. Silliman, B.R. Salt marshes. Curr. Biol. 2014, 24, R348-R350. [CrossRef] [PubMed]

10. Teal, J.M. Salt marshes and mud flats. In Encyclopedia of Ocean Sciences; Thorpe, S.A., Turekian, K.K., Eds.; Academic Press: Cambridge, MA, USA, 2008; pp. 43-48.

11. Roman, C.T. Salt marsh vegetation. In Encyclopedia of Ocean Sciences; Thorpe, S.A., Turekian, K.K., Eds.; Academic Press: Cambridge, MA, USA, 2001; pp. 2487-2490.

12. Garbutt, A.; de Groot, A.; Smit, C.; Pétillon, J. European salt marshes: Ecology and conservation in a changing world. J. Coast. Conserv. 2017, 21, 405-408. [CrossRef]

13. Davy, A.J. Development and structure of salt marshes: Community patterns in time and space. In Concepts and Controversies in Tidal Marsh Ecology; Weinstein, M.P., Kreeger, D.A., Eds.; Springer: Dordrecht, The Netherlands, 2005; pp. 137-156.

14. Pennings, S.C.; Callaway, R.M. Salt marsh plant zonation: The relative importance of competition and physical factors. Ecology 1992, 73, 681-690. [CrossRef]

15. Pomeroy, L.R.; Darley, W.M.; Dunn, E.L.; Gallagher, J.L.; Haines, E.B.; Whitney, D.M. Primary production. In The Ecology of Salt Marsh; Pomeroy, L.R., Wiegert, R.G., Eds.; Springer-Verlag: New York, NY, USA, 1981; pp. 39-67. 
16. Howarth, R.W.; Hobbie, J.E. The Regulation of Decomposition and Heterotrophic Microbial Activity in Salt Marsh Soils: A Review; Kennedy, V.S., Ed.; Academic Press: Cambridge, MA, USA, 1982.

17. Long, S.P.; Mason, C.F. Saltmarsh Ecology; Blackie: Glasgow, UK, 1983.

18. Valiela, I.; Teal, J.M.; Allen, S.D.; Van Etten, R.; Goehringer, D.; Volkmann, S. Decomposition in salt marsh ecosystems: The phases and major factors affecting disappearance of above-ground organic matter. J. Exp. Mar. Biol. Ecol. 1985, 89, 29-54. [CrossRef]

19. Pomeroy, L.R.; Imberger, J. The physical and chemical environment. In The Ecology of Salt Marsh; Pomeroy, L.R., Weigert, R.G., Eds.; Springer-Verlag: New York, NY, USA, 1981; pp. 21-36.

20. Gessner, R.V.; Goos, R.D. Fungi from decomposing Spartina alterniflora. Can. J. Bot. 1973, 51, 51-55. [CrossRef]

21. Gessner, R.V.; Goos, R.D.; Sieburth, J.M.N. The fungal microcosm of the internodes of Spartina alterniflora. Mar. Biol. 1972, 16, 269-273. [CrossRef]

22. Benner, R.; Newell, S.Y.; Maccubbin, A.E.; Hodson, R.E. Relative contributions of bacteria and fungi to rates of degradation of lignocellulosic detritus in salt-marsh sediments. Appl. Environ. Microbiol. 1984, 48, 36-40. [CrossRef]

23. Bergbauer, M.; Newell, S.Y. Contribution to lignocellulose degradation and DOC formation from a salt marsh macrophyte by the ascomycete Phaeosphaeria spartinicola. FEMS Microbiol. Ecol. 1992, 86, 34-348. [CrossRef]

24. Benner, R.; Maccubbin, A.E.; Hodson, R.E. Preparation, characterization, and microbial degradation of specifically radiolabeled [C] lignocelluloses from marine and freshwater macrophytes. Appl. Environ. Microbiol. 1984, 47, 381-389. [CrossRef]

25. Lyons, J.I.; Alber, M.; Hollibaugh, J.T. Ascomycete fungal communities associated with early decaying leaves of Spartina spp. from central California estuaries. Oecologia 2010, 162, 435-442. [CrossRef]

26. Maccubbin, A.E.; Hodson, R.E. Mineralization of detrital lignocelluloses by salt marsh sediment microflora. Appl. Environ. Microbiol. 1980, 40, 735-740. [CrossRef]

27. Newell, S.Y.; Porter, D. Microbial secondary production from salt marsh-grass shoots, and its known and potential fates. In Concepts and Controversies in Tidal Marsh Ecology; Weinstein, M.P., Kreeger, D.A., Eds.; Springer: Dordrecht, The Netherlands, 2005; pp. 159-185.

28. Newell, S.Y.; Fallon, R.D.; Miller, J.D. Decomposition and microbial dynamics for standing, naturally positioned leaves of the salt-marsh grass Spartina alterniflora. Mar. Biol. 1989, 101, 471-481. [CrossRef]

29. Hernández, E.G.; Baraza, E.; Smit, C.; Berg, M.P.; Salles, J.F. Salt marsh elevation drives root microbial composition of the native invasive grass Elytrigia atherica. Microorganisms 2020, 8, 1619. [CrossRef] [PubMed]

30. Wang, M.; Li, E.; Liu, C.; Jousset, A.; Salles, J.F. Functionality of root-associated bacteria along a salt marsh primary succession. Front. Microbiol. 2017, 8, 2102. [CrossRef] [PubMed]

31. Calado, M.d.L.; Carvalho, L.; Barata, M.; Pang, K.L. Potential roles of marine fungi in the decomposition process of standing stems and leaves of Spartina maritima. Mycologia 2019, 111, 371-383. [CrossRef] [PubMed]

32. Kohlmeyer, J.; Volkmann-Kohlmeyer, B. Fungi on Juncus and Spartina: New marine species of Anthostomella, with a list of marine fungi known on Spartina. Mycol. Res. 2002, 106, 365-374. [CrossRef]

33. Wijayawardene, N.N.; Hyde, K.D.; Al-ani, L.K.T.; Tedersoo, L.; Haelewaters, D.; Rajeshkumar, K.C.; Zhao, R.; Aptroot, A.; Leontyev, D.V.; Ramesh, K.; et al. Outline of Fungi and fungus-like taxa. Mycosphere 2020, 11, 1-367. [CrossRef]

34. Calado, M.d.L.; Barata, M. Salt marsh fungi. In Marine Fungi and Fungal-like Organisms; Jones, E.B.G., Pang, K.L., Eds.; Walter de Gruyter GmbH \& Co. KG: Berlin, Germany, 2012; pp. 345-381.

35. Kohlmeyer, J.; Kohlmeyer, E. Marine Mycology: The Higher Fungi; Academic Press: New York, NY, USA, 1979.

36. Gessner, R.V.; Kohlmeyer, J. Geographical distribution and taxonomy of fungi from salt marsh Spartina. Can. J. Bot. 1976, 54, 2023-2037. [CrossRef]

37. Quattrocchi, U. CRC World Dictionary of Grasses; CRC Press: Boca Raton, FL, USA, 2006.

38. Apinis, A.E.; Chesters, C.G.C. Ascomycetes of some salt marshes and sand dunes. Trans. Br. Mycol. Soc. 1964, 47, 419-435. [CrossRef]

39. Van Ryckegem, G.; Verbeken, A. Fungal ecology and succession on Phragmites australis in a brackish tidal marsh. I. Leaf sheaths. Fungal Divers. 2005, 19, 157-187.

40. Van Ryckegem, G.; Verbeken, A. Fungal ecology and succession on Phragmites australis in a brackish tidal marsh. II. Stems. Fungal Divers. 2005, 20, 209-233.

41. Poon, M.O.K.; Hyde, K.D. Biodiversity of intertidal estuarine fungi on Phragmites at Mai Po Marshes, Hong Kong. Bot. Mar. 1998, 41,141-155. [CrossRef]

42. Kohlmeyer, J.; Volkmann-Kohlmeyer, B. Fungi on Juncus roemerianus. 7. Tiarosporella halmyra sp. nov. Mycotaxon 1996, 59, 79-83.

43. Fell, J.W.; Hunter, I.L. Fungi associated with the decomposition of the Black Rush, Juncus roemerianus, in South Florida. Mycologia 1979, 71, 322-342. [CrossRef]

44. United State Department of Agriculture Crops Research Division. Index of Plant Diseases in the United States: Agriculture Handbook; USDA: Washington, DC, USA, 1960.

45. Brunaud, P. Champignons nouvellement obsrevés aux environs de Saintes, Charente-inférieure. J. Hist. Nat. Bord Sud. Oust. Bord. $1888,7,4$.

46. Lobik, A.I. Materialen zur Mykoflora des Terskikreises. Morbi Plant. Leningr. 1928, 17, 157-199.

47. Elíades, L.A.; Voget, C.E.; Arambarri, A.M.; Cabello, M.N. Fungal communities on decaying saltgrass (Distichlis spicata) in Buenos Aires province (Argentina). Sydowia 2007, 59, 227-234. 
48. Miller, J.D.; Whitney, N.J. Fungi of the Bay of Fundy V: Fungi from living species of Spartina Schreber. Proc. Nov. Scotian Inst. Sci. 1983, 33, 75-83.

49. Goodman, P.J. The possible role of pathogenic fungi in die-back of Spartina townsendii agg. Trans. Br. Mycol. Soc. 1959, 42, 409-415. [CrossRef]

50. Van Ryckegem, G.; Gessner, M.O.; Verbeken, A. Fungi on leaf blades of Phragmites australis in a brackish tidal marsh: Diversity, succession, and leaf decomposition. Microb. Ecol. 2007, 53, 600-611. [CrossRef]

51. Kohlmeyer, J.; Volkmann-Kohlmeyer, B.; Eriksson, O.E. Fungi on Juncus roemerianus 12. Two new species of Mycosphaerella and Paraphaeosphaeria (Ascomycotina). Bot. Mar. 1999, 42, 505-511. [CrossRef]

52. Borse, B.D.; Bhat, D.; Borse, K.; Tuwar, A.; Pawar, N. Marine fungi of India (Monograph); Broadway Book Centre: Panaji, India, 2012.

53. Kohlmeyer, J.; Kohlmeyer, E. Bermuda marine fungi. Trans. Br. Mycol. Soc. 1977, 68, 207-219. [CrossRef]

54. Barata, M. Fungi on the halophyte Spartina maritima in salt marshes. In Fungi in Marine Environments; Hyde, K.D., Ed.; Fungal Diversity Press: Hong Kong, China, 2002; pp. 179-193.

55. Walker, A.K.; Campbell, J. Marine fungal diversity: A comparison of natural and created salt marshes of the north-central Gulf of Mexico. Mycologia 2010, 102, 513-521. [CrossRef]

56. Buchan, A.; Newell, S.Y.; Moreta, J.I.L.; Moran, M.A. Analysis of internal transcribed spacer (ITS) regions of rRNA genes in fungal communities in a southeastern U.S. salt marsh. Microb. Ecol. 2002, 43, 329-340. [CrossRef] [PubMed]

57. Buchan, A.; Newell, S.Y.; Butler, M.; Biers, E.J.; Hollibaugh, J.T.; Moran, M.A. Dynamics of bacterial and fungal communities on decaying salt marsh grass. Appl. Environ. Microbiol. 2003, 69, 6676-6687. [CrossRef] [PubMed]

58. Walker, A.K. Marine Fungi of U.S. GULF of Mexico Barrier Island Beaches: Biodiversity and Sampling Strategy; The University of Southern Mississippi: Hattiesburg, MS, USA, 2012.

59. Calado, M.d.L.; Carvalho, L.; Pang, K.L.; Barata, M. Diversity and ecological characterization of sporulating higher filamentous marine fungi associated with Spartina maritima (Curtis) Fernald in two Portuguese salt marshes. Microb. Ecol. 2015, 70, 612-633. [CrossRef]

60. Kohlmeyer, J.; Volkmann-Kohlmeyer, B.; Eriksson, O.E. Fungi on Juncus roemerianus. 11. More new ascomycetes. Can. J. Bot. 1998, 76, 467-477. [CrossRef]

61. Gessner, R.V.; Goos, R.D. Fungi from Spartina alterniflora in Rhode Island. Mycologia 1973, 65, 1296-1301. [CrossRef]

62. Hansford, C.G. Australian Fungi. II. New species and revisions. Proc. Linn. Soc. N. S. W. 1954, 79, 97-141.

63. Barata, M. Marine fungi from Mira River salt marsh in Portugal. Rev. Iberoam. Micol. 2006, 23, 179-184. [CrossRef]

64. Peña, N.I.; Arambarri, A.M. Hongos marinos lignícolas de la laguna costera de Mar Chiquita (provincia de Buenos Aires, Argentina). L. Ascomycotina y Deuteromycotina sobre Spartina densiflora. Darwiniana 1998, 35, 61-67.

65. Jones, E.B.G. Marine fungi. I. Trans. Br. Mycol. Soc. 1962, 45, 93-114. [CrossRef]

66. Kohlmeyer, J.; Volkmann-Kohlmeyer, B.; Eriksson, O.E. Fungi on Juncus roemerianus. 8. New bitunicate ascomycetes. Can. J. Bot. 1996, 74, 1830-1840. [CrossRef]

67. Johnson, T.W.; Hughes, G.C. Robillarda phragmitis Cunnell in estuarine waters. Trans. Br. Mycol. Soc. 1960, 43, 523-524. [CrossRef]

68. Seymour, A.B. Host Index of the Fungi of North America; Harvard University Press: Cambridge, MA, USA, 1929.

69. Saccardo, P.A. Fungi Gallici lecti a cl. viris P. Brunaud, Abb. Letendre, A. Malbranche, J. Therry, vel editi in Mycotheca Gallica C. Roumeguèri. Series II. Michelia 1880, 2, 39-135.

70. Ahmad, S. Contributions to the fungi of West Pakistan. VI. Biol. Lahore 1967, 13, 15-42.

71. Johnson, T.W.; Sparrow, F.K. Fungi in Oceans and Estuaries; Cramer: Weinheim, Germany, 1961.

72. Jones, E.B.G. Marine fungi: II. Ascomycetes and deuteromycetes from submerged wood and drift Spartina. Trans. Br. Mycol. Soc. 1963, 46, 135-144. [CrossRef]

73. Gessner, R.V. Seasonal occurrence and distribution of fungi associated with Spartina alterniflora from a Rhode Island estuary. Mycologia 1977, 69, 477-491. [CrossRef]

74. Gessner, R.V. Degradative enzyme production by salt-marsh fungi. Bot. Mar. 1980, 23, 133-139. [CrossRef]

75. Jaap, O. Weitere Beiträge zur Pilzflora der nordfriesischen Inseln. Schr. Des Nat. Ver. Für Schleswig-Holst. 1907, 14, 15-33.

76. Kohlmeyer, J.; Volkmann-Kohlmeyer, B.; Eriksson, O.E. Fungi on Juncus roemerianus 9. New obligate and facultative marine ascomycotina. Bot. Mar. 1997, 40, 291-300. [CrossRef]

77. Kohlmeyer, J.; Volkmann-Kohlmeyer, B.; Eriksson, O.E. Fungi on Juncus roemerianus. New marine and terrestrial ascomycetes. Mycol. Res. 1996, 100, 393-404. [CrossRef]

78. Kohlmeyer, J.; Volkmann-Kohlmeyer, B.; Eriksson, O.E. Fungi on Juncus roemerianus 2. New dictyosporous Ascomycetes. Bot. Mar. 1995, 38, 165-174. [CrossRef]

79. Devadatha, B.; Calabon, M.S.; Abeywickrama, P.D.; Hyde, K.D.; Jones, E.B.G. Molecular data reveals a new holomorphic marine fungus, Halobyssothecium estuariae, and the asexual morph of Keissleriella phragmiticola. Mycology 2020, 11, 167-183. [CrossRef] [PubMed]

80. Johnson, T.W. Marine Fungi. I. Leptosphaeria and Pleospora. Mycologia 1956, 48, 495-505. [CrossRef]

81. Wagner, D. Ecological studies on Leptospheria discors, a graminicolous fungus of salt marshes. Nov. Hedwig. 1969, 18, 383-396.

82. Webber, E.E. Marine ascomycetes from New England. Bull. Torrey Bot. Club 1970, 97, 119-120. [CrossRef]

83. Dayarathne, M.C.; Wanasinghe, D.N.; Jones, E.B.G.; Chomnunti, P.; Hyde, K.D. A novel marine genus, Halobyssothecium (Lentitheciaceae) and epitypification of Halobyssothecium obiones comb. nov. Mycol. Prog. 2018, 17, 1161-1171. [CrossRef] 
84. Saccardo, P.A. Sylloge fungorum Omnium Hucusque Cognitorum; R. Friedländer \& Sohn.: Berlin, Germany, 1883 ; Volume 2.

85. Calabon, M.S.; Jones, E.B.G.; Hyde, K.D.; Boonmee, S.; Tibell, S.; Tibell, L.; Pang, K.L.; Phookamsak, R. Phylogenetic assessment and taxonomic revision of Halobyssothecium and Lentithecium (Lentitheciaceae, Pleosporales). Mycol. Prog. 2021, 20, 701-720. [CrossRef]

86. Van Ryckegem, G.; Aptroot, A. A new Massarina and a new Wettsteinina (Ascomycota) from freshwater and tidal reeds. Nov. Hedwig. 2001, 73, 161-166. [CrossRef]

87. Tibell, S.; Tibell, L.; Pang, K.L.; Calabon, M.S.; Jones, E.B.G. Marine fungi of the Baltic Sea. Mycology 2020, 11, 195-213. [CrossRef] [PubMed]

88. Li, G.J.; Hyde, K.D.; Zhao, R.L.; Hongsanan, S.; Abdel-Aziz, F.A.; Abdel-Wahab, M.A.; Alvarado, P.; Alves-Silva, G.; Ammirati, J.F.; Ariyawansa, H.A.; et al. Fungal diversity notes 253-366: Taxonomic and phylogenetic contributions to fungal taxa. Fungal Divers. 2016, 78, 1-237. [CrossRef]

89. Ellis, J.B.; Everhart, B.M. New Fungi. J. Mycol. 1885, 1, 42-44. [CrossRef]

90. Ellis, J.B.; Everhart, B.M. The North American Pyrenomycetes. A Contribution to Mycologic Botany; Ellis \& Everhart: Newfield, NJ, USA, 1892.

91. Lucas, M.T. Culture studies on portuguese species of Leptosphaeria I. Trans. Br. Mycol. Soc. 1963, 46, 361-367. [CrossRef]

92. Webber, E.E. Fungi from a Massachusetts salt marsh. Trans. Am. Microsc. Soc. 1966, 85, 556-558. [CrossRef]

93. Dennis, R.W.G. British Ascomycetes; J. Cramer: Lehre, Germany, 1968.

94. Poli, A.; Vizzini, A.; Prigione, V.; Varese, G.C. Basidiomycota isolated from the Mediterranean Sea-Phylogeny and putative ecological roles. Fungal Ecol. 2018. [CrossRef]

95. Hansford, C.G. Australian fungi IV. New species and revisions (cont'd). Proc. Linn. Soc. N. S. W. 1957, 82, $209-229$.

96. Hyde, K.D.; Hongsanan, S.; Jeewon, R.; Bhat, D.J.; McKenzie, E.H.C.; Jones, E.B.G.; Phookamsak, R.; Ariyawansa, H.A.; Boonmee, S.; Zhao, Q.; et al. Fungal diversity notes 367-490: Taxonomic and phylogenetic contributions to fungal taxa. Fungal Divers. 2016, 80, 1-270. [CrossRef]

97. Kohlmeyer, J.; Volkmann-Kohlmeyer, B.; Eriksson, O.E. Fungi on Juncus roemerianus. 4. New marine ascomycetes. Mycologia 1995, 87, 532-542. [CrossRef]

98. Kohlmeyer, J.; Volkmann-Kohlmeyer, B. Fungi on Juncus roemerianus. 14. Three new coelomycetes, including Floricola, anam.-gen. nov. Bot. Mar. 2000, 43, 385-392. [CrossRef]

99. Sydow, H. Mycotheca germanica. Fasc. XX-XXI. Ann. Mycol. 1911, 9, 554-558.

100. Dayarathne, M.; Jones, E.; Maharachchikumbura, S.; Devadatha, B.; Sarma, V.; Khongphinitbunjong, K.; Chomnunti, P.; Hyde, K. Morpho-molecular characterization of microfungi associated with marine based habitats. Mycosphere 2020, 11, 1-188. [CrossRef]

101. Hyde, K.D.; Chaiwan, N.; Norphanphoun, C.; Boonmee, S.; Camporesi, E.; Chethana, K.W.T.; Dayarathne, M.C.; de Silva, N.I.; Dissanayake, A.J.; Ekanayaka, A.H.; et al. Mycosphere notes 169-224. Mycosphere 2018, 9, 271-430. [CrossRef]

102. Wanasinghe, D.N.; Hyde, K.D.; Jeewon, R.; Crous, P.W.; Wijayawardene, N.N.; Jones, E.B.G.; Bhat, D.J.; Phillips, A.J.L.; Groenewald, J.Z.; Dayarathne, M.C.; et al. Phylogenetic revision of Camarosporium (Pleosporineae, Dothideomycetes) and allied genera. Stud. Mycol. 2017, 87, 207-256. [CrossRef]

103. Grove, W.B. British Stem- and Leaf-Fungi Vol. II; Cambridge University Press: Cambridge, UK, 1937.

104. Kohlmeyer, J.; Volkmann-Kohlmeyer, B. Atrotorquata and Loratospora: New ascomycete genera on Juncus roemerianus. Syst. Ascomycetum 1993, 12, 7-22.

105. Kohlmeyer, J.; Volkmann-Kohlmeyer, B.; Tsui, C.K.M. Fungi on Juncus roemerianus. 17. New ascomycetes and the hyphomycete genus Kolletes gen. nov. Bot. Mar. 2005, 48, 306-317. [CrossRef]

106. Yusoff, M.; Moss, S.T.; Jones, E.B.G. Ascospore ultrastructure of Pleospora gaudefroyi (Pleosporaceae, Loculoascomycetes, Ascomycotina). Can. J. Bot. 1994, 72, 1-6. [CrossRef]

107. Webster, J.; Lucas, M.T. Observations on British species of Pleospora. II. Trans. Br. Mycol. Soc. 1961, 44, 417-436. [CrossRef]

108. Sutton, B.C.; Pirozynski, K.A. Notes on British microfungi. I. Trans. Br. Mycol. Soc. 1963, 46, 505-522. [CrossRef]

109. Spegazzini, C. Mycetes Argentinenses. Series IV. An. del Mus. Nac. Hist. Nat. Buenos Aires. Ser. 3 1909, 12, $257-458$.

110. Kohlmeyer, J.; Volkmann-Kohlmeyer, B. Fungi on Juncus roemerianus. 6. Glomerobolus gen. nov., the first ballistic member of Agonomycetales. Mycologia 1996, 88, 328-337. [CrossRef]

111. Cantrell, S.A.; Hanlin, R.T.; Newell, S.Y. A new species of Lachnum on Spartina alterniflora. Mycotaxon 1996, 57, $479-485$.

112. Ellis, J.B.; Everhart, B.M. New species of fungi from various localities. J. Mycol. 1888, 4, 121-124. [CrossRef]

113. Kohlmeyer, J.; Baral, H.-O.; Volkmann-Kohlmeyer, B. Fungi on Juncus roemerianus. 10. A new Orbilia with ingoldian anamorph. Mycologia 1998, 90, 303-309. [CrossRef]

114. Seaver, F.J. The North American Cup-Fungi (Inoperculates); Lancaster Press Inc.: Lancaster, PA, USA, 1951.

115. Wong, M.K.M.; Goh, T.K.; Hyde, K.D. A new species of Phragmitensis (ascomycetes) from senescent culms of Phragmites australis. Fungal Divers. 1999, 2, 175-180.

116. Kohlmeyer, J.; Volkmann Kohlmeyer, B.; Eriksson, O.E. Fungi on Juncus roemerianus. 3. New ascomycetes. Bot. Mar. 1995, 38, 175-186. [CrossRef]

117. Kohlmeyer, J.; Volkmann-Kohlmeyer, B. Fungi on Juncus roemerianus. 16. More new coelomycetes, including Tetranacriella, gen. nov. Bot. Mar. 2001, 44, 147-156. [CrossRef] 
118. Kohlmeyer, J.; Volkmann-Kohlmeyer, B. Two new genera of Ascomycotina from saltmarsh Juncus. Syst. Ascomycetum 1993, 11, 95-106.

119. Loveless, A.R.; Peach, J.M. Evidence from ascospores for host restriction in Claviceps purpurea. Trans. Br. Mycol. Soc. 1986, 86, 603-610. [CrossRef]

120. Loveless, A.R. Conidial evidence for host restriction in Claviceps purpurea. Trans. Br. Mycol. Soc. 1971, 56, 419-434. [CrossRef]

121. Sprague, R. Septoria disease of Gramineae in western United States. Oregon State Monogr. Stud. Bot. 1944, 6, 1-151.

122. Spegazzini, C. Fungi Argentini additis nonnullis brasiliensibus montevidensibusque. An. la Soc. Cient. Argent. 1882, $13,60-64$.

123. Peach, J.M.; Loveless, A.R. A comparison of two methods of inoculating Triticum aestivum with spore suspensions of Claviceps purpurea. Trans. Br. Mycol. Soc. 1975, 64, 328-331. [CrossRef]

124. Eleuterius, L.N.; Meyers, S.P. Claviceps purpurea on Spartina in coastal marshes. Mycologia 1974, 66, 978-986. [CrossRef]

125. Sprague, R. Diseases of Cereals and Grasses in North America: Fungi Except Smuts and Rusts; Ronald Press Company: New York, NY, USA, 1950.

126. Mantle, P.G. Development of alkaloid production in vitro by a strain of Claviceps purpurea from Spartina townsendii. Trans. Br. Mycol. Soc. 1969, 52, 381-392. [CrossRef]

127. Moberley, D.G. Taxonomy and distribution of the genus Spartina. Iowa State J. Sci. 1956, 30, 471-574.

128. Saccardo, P.A. Sylloge Fungorum Omnium Hucusque Cognitorum; R. Friedländer \& Sohn.: Berlin, Germany, 1886 ; Volume 4.

129. Abdullah, S.K.; Abdulkadder, M.A.; Goos, R.D. Basramyces marinus nom.nov. (hyphomycete) from southern marshes of Iraq. Int. J. Mycol. Lichenol. 1989, 4, 181-186.

130. Abdel-Wahab, M.A.; Pang, K.L.; Nagahama, T.; Abdel-Aziz, F.A.; Jones, E.B.G. Phylogenetic evaluation of anamorphic species of Cirrenalia and Cumulospora with the description of eight new genera and four new species. Mycol. Prog. 2010, 9, 537-558. [CrossRef]

131. Johnson, T.W. Marine Fungi. IV. Lulworthia and Ceriosporopsis. Mycologia 1958, 50, 151-163. [CrossRef]

132. Mounce, I.; Diehl, W.W. A new Ophiobolus on eelgrass. Can. J. Res. 1934, 11, 242-246. [CrossRef]

133. Johnson, T.W. Marine fungi. II. Ascomycetes and Deuteromycetes from submerged wood. Mycologia 1956, 48, 841-851. [CrossRef]

134. Lloyd, L.S.; Wilson, I.M. Development of the perithecium in Lulworthia medusa (Ell. \& Ev.) Cribb \& Cribb, a saprophyte on Spartina townsendii. Trans. Br. Mycol. Soc. 1962, 45, 359-372. [CrossRef]

135. Kohlmeyer, J.; Volkmann-Kohlmeyer, B. Fungi on Juncus roemerianus. 1. Trichocladium medullare sp. nov. Mycotaxon 1995, 53, 349-353.

136. Volkmann-Kohlmeyer, B.; Kohlmeyer, J. A new Aniptodera (Ascomycotina) from saltmarsh Juncus. Bot. Mar. 1994, 37, 109-114. [CrossRef]

137. Gessner, R.V. Spartina alterniflora seed fungi. Can. J. Bot. 1978, 56, 2942-2947. [CrossRef]

138. Kohlmeyer, J.; Kohlmeyer, E. Icones Fungorum Maris. Fasc. 1-9; J. Cramer: Weinheim/Lehre, Germany, 1967.

139. Jones, E.B.G. Haligena spartinae sp. nov., a pyrenomycete on Spartina townsendii. Trans. Br. Mycol. Soc. 1962, 45, 245-248. [CrossRef]

140. Liu, J.K.; Hyde, K.D.; Jones, E.B.G.; Ariyawansa, H.A.; Bhat, D.J.; Boonmee, S.; Maharachchikumbura, S.S.N.; McKenzie, E.H.C.; Phookamsak, R.; Phukhamsakda, C.; et al. Fungal diversity notes 1-110: Taxonomic and phylogenetic contributions to fungal species. Fungal Divers. 2015, 72, 1-197. [CrossRef]

141. Orton, C.R. Graminicolous species of Phyllachora in North America. Mycologia 1944, 36, 18-53. [CrossRef]

142. Kohlmeyer, J.; Volkmann-Kohlmeyer, B. Octopodotus stupendus gen. \& sp. nov. and Phyllachora paludicola sp. nov., two marine fungi from Spartina alterniflora. Mycologia 2003, 95, 117-123. [CrossRef]

143. Kohlmeyer, J.; Volkmann-Kohlmeyer, B. Aropsiclus nom. nov. (Ascomycotina) to replace Sulcospora Kohlm. \& Volk.-Kohlm. Syst. Ascomycetum 1994, 13, 24.

144. Spooner, B.M. New records and species of British microfungi. Trans. Br. Mycol. Soc. 1981, 76, 265-301. [CrossRef]

145. Dayarathne, M.C.; Wanasinghe, D.N.; Devadatha, B.; Abeywickrama, P.; Gareth Jones, E.B.; Chomnunti, P.; Sarma, V.V.; Hyde, K.D.; Lumyong, S.; Mckenzie, E.H.C. Modern taxonomic approaches to identifying diatrypaceous fungi from marine habitats, with a novel genus Halocryptovalsa Dayarathne \& K.D.Hyde, gen. Nov. Cryptogam. Mycol. 2020, 41, 21-67. [CrossRef]

146. Johnson, T.W.J.; Gold, H.S. Didymosamarospora, a new genus of fungi from fresh and marine waters. J. Elisha Mitchell Sci. Soc. 1957, 73, 103-108.

147. Kohlmeyer, J.; Volkmann-Kohlmeyer, B. Fungi on Juncus roemerianus: New coelomycetes with notes on Dwayaangam junci. Mycol. Res. 2001, 105, 500-505. [CrossRef]

148. Kohlmeyer, J.; Volkmann-Kohlmeyer, B. Fungi on Juncus roemerianus. 13. Hyphopolynema juncatile sp. nov. Mycotaxon 1999, 70, 489-495.

149. Kohlmeyer, J.; Kohlmeyer, E. Marine fungi from tropical America and Africa. Mycologia 1971, 63, 831-861. [CrossRef]

150. Kohlmeyer, J.; Kohlmeyer, E. New marine fungi from mangroves and trees along eroding shorelines. Nov. Hedwig. 1965, 9, 89-104.

151. Ellis, J.B.; Everhart, B.M. New species of North American fungi from various localities. Proc. Acad. Nat. Sci. Phila. 1893, 45, 128-172. [CrossRef]

152. Cummins, G.B. The Rust Fungi of Cereals, Grasses and Bamboos; Springer: New York, NY, USA; Berlin/Heidelberg, Germany, 1971.

153. Orton, C.R. Manual of the Rusts in United States and Canada; Purdue Research Foundation: Lafayette, IN, USA, 1934.

154. von Thümen, F. Fungi Egyptiaci, Ser. III. Flora (Regensbg.) 1880, 63, 477-479. 
155. McAlpine, D. The Smuts of Australia, Their Structure, Life History, Treatment, and Classification; Department of Agriculture of Victoria, Melbourn, Australia: Melbourne, VIC, Australia, 1910.

156. Bauer, R.; Lutz, M.; Piatek, M.; Vánky, K.; Oberwinkler, F. Flamingomyces and Parvulago, new genera of marine smut fungi (Ustilaginomycotina). Mycol. Res. 2007, 111, 1199-1206. [CrossRef] [PubMed]

157. Dennis, R.W.G. Fungi of Ammophila arenaria in Europe. Rev. Biol. 1983, 12, 15-48.

158. Treigienè, A. Fungi associated with Ammophila arenaria in Lithuania and taxonomical notes on some species. Bot. Lith. 2011, 17, 47-53.

159. Rodríguez-Echeverría, S.; Hol, W.H.G.; Freitas, H.; Eason, W.R.; Cook, R. Arbuscular mycorrhizal fungi of Ammophila arenaria (L.) Link: Spore abundance and root colonisation in six locations of the European coast. Eur. J. Soil Biol. 2008, 44, 30-36. [CrossRef]

160. Alva, P.; McKenzie, E.H.C.; Pointing, S.B.; Pena-Murala, R.; Hyde, K.D. Do sea grasses harbour endophytes? In Fungi in Marine Environments; Hyde, K.D., Ed.; Fungal Diversity Press: Hong Kong, China, 2002; pp. 167-178.

161. Wagner, D.T. Developmental morphology of Leptosphaeria discors (Saccardo and Ellis) Saccardo and Ellis. Nov. Hedwig. 1965, 9 , 45-61.

162. Meyers, S.P.; Ahearn, D.G.; Alexander, S.K.; Cook, W.L. Pichia spartinae, a dominant yeast of the Spartina salt marsh. Dev. Ind. Microbiol. 1975, 16, 261-267.

163. Jones, E.B.G.; Pang, K.L.; Abdel-Wahab, M.A.; Scholz, B.; Hyde, K.D.; Boekhout, T.; Ebel, R.; Rateb, M.E.; Henderson, L.; Sakayaroj, J.; et al. An online resource for marine fungi. Fungal Divers. 2019, 96, 347-433. [CrossRef]

164. Mohamed, D.J.; Martiny, J.B.H. Patterns of fungal diversity and composition along a salinity gradient. ISME J. 2011, 5, 379-388. [CrossRef]

165. Stoeck, T.; Epstein, S. Novel eukaryotic lineages inferred from small-subunit rRNA analyses of oxygen-depleted marine environments. Appl. Environ. Microbiol. 2003, 69, 2657-2663. [CrossRef]

166. Dini-Andreote, F.; Pylro, V.S.; Baldrian, P.; Van Elsas, J.D.; Salles, J.F. Ecological succession reveals potential signatures of marine-terrestrial transition in salt marsh fungal communities. ISME J. 2016, 10, 1984-1997. [CrossRef]

167. D'entremont, T.W. Saltmarsh Sediment Fungal Communities and Arbuscular Mycorrhizal Fungi in Sporobolus Pumilus (Roth) (Poaceae) (Spartina Patens) of the Minas Basin, Nova Scotia; Identification, Abundance and Role in Restoration. Ph.D. Thesis, Acadia University, Wolfville, NS, Canada, 2019.

168. Khalmuratova, I.; Kim, H.; Nam, Y.J.; Oh, Y.; Jeong, M.J.; Choi, H.R.; You, Y.H.; Choo, Y.S.; Lee, I.J.; Shin, J.H.; et al. Diversity and plant growth promoting capacity of endophytic fungi associated with halophytic plants from the west coast of Korea. Mycobiology 2015, 43, 373-383. [CrossRef] [PubMed]

169. You, Y.H.; Yoon, H.; Kang, S.M.; Shin, J.H.; Choo, Y.S.; Lee, I.J.; Lee, J.M.; Kim, J.G. Fungal diversity and plant growth promotion of endophytic fungi from six halophytes in Suncheon Bay. J. Microbiol. Biotechnol. 2012, 22, 1549-1556. [CrossRef] [PubMed]

170. Khalmuratova, I.; Choi, D.H.; Woo, J.R.; Jeong, M.J.; Oh, Y.; Kim, Y.G.; Lee, I.J.; Choo, Y.S.; Kim, J.G. Diversity and plant growth-promoting effects of fungal endophytes isolated from salt-tolerant plants. J. Microbiol. Biotechnol. 2020, 30, $1680-1687$. [CrossRef] [PubMed]

171. Kandalepas, D.; Blum, M.J.; Van Bael, S.A. Shifts in symbiotic endophyte communities of a foundational salt marsh grass following oil exposure from the deepwater horizon oil spill. PLoS ONE 2015, 10, e0122378. [CrossRef] [PubMed]

172. Maciá-Vicente, J.G.; Jansson, H.B.; Abdullah, S.K.; Descals, E.; Salinas, J.; Lopez-Llorca, L.V. Fungal root endophytes from natural vegetation in Mediterranean environments with special reference to Fusarium spp. FEMS Microbiol. Ecol. 2008, 64, 90-105. [CrossRef]

173. You, Y.-H.; Yoon, H.-J.; Woo, J.-R.; Seo, Y.-G.; Kim, M.-A.; Lee, G.-M.; Kim, J.-G. Diversity of endophytic fungi from the roots of halophytes growing in Go-chang salt marsh. Korean J. Mycol. 2012, 40, 86-92. [CrossRef]

174. Khalmuratova, I.; Choi, D.H.; Yoon, H.J.; Yoon, T.M.; Kim, J.G. Diversity and plant growth promotion of fungal endophytes in five halophytes from the Buan salt marsh. J. Microbiol. Biotechnol. 2021, 31, 408-418. [CrossRef]

175. Kalyanasundaram, I.; Nagamuthu, J.; Muthukumaraswamy, S. Antimicrobial activity of endophytic fungi isolated and identified from salt marsh plant in Vellar Estuary. J. Microbiol. Biotechnol. 2015, 7, 13-20. [CrossRef]

176. Elmer, W.H.; Marra, R.E. New species of Fusarium associated with dieback of Spartina alterniflora in Atlantic salt marshes. Mycologia 2011, 103, 806-819. [CrossRef]

177. Alber, M.; Swenson, E.M.; Adamowicz, S.C.; Mendelssohn, I.A. Salt marsh dieback: An overview of recent events in the US. Estuar. Coast. Shelf Sci. 2008, 80,1-11. [CrossRef]

178. Elmer, W.H. Pathogenic microfungi associated with Spartina in salt marshes. In Biology of Microfungi; Li, D.-W., Ed.; Springer: New York, NY, USA; Cham: Switzerland, 2016; pp. 615-630.

179. Govers, L.L.; in'T Veld, W.A.M.; Meffert, J.P.; Bouma, T.J.; van Rijswick, P.C.J.; Heusinkveld, J.H.T.; Orth, R.J.; van Katwijk, M.M.; van der Heide, T. Marine Phytophthora species can hamper conservation and restoration of vegetated coastal ecosystems. Proc. R. Soc. B Biol. Sci. 2016, 283, 20160812. [CrossRef] [PubMed]

180. Fisher, A.J.; DiTomaso, J.M.; Gordon, T.R.; Aegerter, B.J.; Ayres, D.R. Salt marsh Claviceps purpurea in native and invaded Spartina marshes in Northern California. Plant Dis. 2007, 91, 380-386. [CrossRef] [PubMed]

181. Raybould, A.F.; Gray, A.J.; Clarke, R.T. The long-term epidemic of Claviceps purpurea on Spartina anglica in Poole Harbour: Pattern of infection, effects on seed production and the role of Fusarium Heterosporum. New Phytol. 1998, 138, 497-505. [CrossRef] 
182. Hyde, K.D.; Norphanphoun, C.; Chen, J.; Dissanayake, A.J.; Doilom, M.; Hongsanan, S.; Jayawardena, R.S.; Jeewon, R.; Perera, R.H.; Thongbai, B.; et al. Thailand's amazing diversity: Up to $96 \%$ of fungi in northern Thailand may be novel. Fungal Divers. 2018, 93, 215-239. [CrossRef]

183. Devadatha, B.; Jones, E.B.G.; Pang, K.L.; Abdel-Wahab, M.A.; Hyde, K.D.; Sakayaroj, J.; Bahkali, A.H.; Calabon, M.S.; Sarma, V.V.; Sutreong, S.; et al. Occurrence and geographical distribution of mangrove fungi. Fungal Divers. 2021, 106, 137-227. [CrossRef]

184. Dong, W.; Wang, B.; Hyde, K.D.; McKenzie, E.H.C.; Raja, H.A.; Tanaka, K.; Abdel-Wahab, M.A.; Abdel-Aziz, F.A.; Doilom, M.; Phookamsak, R.; et al. Freshwater Dothideomycetes. Fungal Divers. 2020, 105, 319-575. [CrossRef]

185. Luo, Z.L.; Hyde, K.D.; Liu, J.K.; Maharachchikumbura, S.S.N.; Jeewon, R.; Bao, D.F.; Bhat, D.J.; Lin, C.G.; Li, W.L.; Yang, J.; et al. Freshwater Sordariomycetes. Fungal Divers. 2019, 99, 451-660. [CrossRef]

186. Hyde, K.D.; Chethana, K.W.T.; Jayawardena, R.S.; Luangharn, T.; Calabon, M.S.; Jones, E.B.G.; Hongsanan, S.; Lumyong, S. The rise of mycology in Asia. Sci. Asia 2020, 46,1-11. [CrossRef]

187. Calabon, M.S.; Jones, E.B.G.; Boonmee, S.; Doilom, M.; Lumyong, S.; Hyde, K.D. Five novel freshwater ascomycetes indicate high undiscovered diversity in lotic habitats in Thailand. J. Fungi 2021, 7, 1-27. [CrossRef]

188. Saintilan, N. Biogeography of Australian saltmarsh plants. Austral Ecol. 2009, 34, 929-937. [CrossRef]

189. Spencer, D.M.; Hickling, V.; Spencer, J.F.T. Yeasts from ponds, streams and salt marsh on the Gower Peninsula, Wales. In Advances in Biotechnology. Proceedings of the Fifth International Yeast Symposium Held in London, Canada, July 20-25; Stewart, G., Russell, I., Eds.; Elsevier: Amsterdam, The Netherlands, 1981; pp. 515-519.

190. Kurtzman, C.P. Saturnospora ahearnii, a new salt marsh yeast from Louisiana. Antonie Van Leeuwenhoek 1991, 60, 31-34. [CrossRef] [PubMed] 